\title{
Intramolecular Aminoazidation of Unactivated Terminal Alkenes by Palladium-Catalyzed Reactions with Hydrogen Peroxide as Oxidant
}

Francesca Foschi, ${ }^{a}$ Camilla Loro, ${ }^{a}$ Roberto Sala, ${ }^{a}$ Julie Oble, ${ }^{b}$ Leonardo Lo Presti, ${ }^{\mathrm{c}}$ Egle M. Beccalli, ${ }^{\mathrm{d}}$ Giovanni Poli ${ }^{\mathrm{b}}$ and Gianluigi Broggini ${ }^{\mathrm{a} *}$

a Dipartimento di Scienza e Alta Tecnologia, Università degli Studi dell'Insubria, Via Valleggio 9, 2210o, Como, Italy

Phone: (+39)-031-238-6443, e-mail: gianluigi.broggini@uninsubria.it

b Sorbonne Université, Faculté des Sciences et Ingénierie, CNRS, Institut Parisien de Chimie Moléculaire, IPCM, 4 place Jussieu, 75005 Paris, France

c Dipartimento di Chimica, Università degli Studi di Milano, Via Golgi 19, 20133, Milano, Italy

d DISFARM, Sezione di Chimica Generale e Organica “A. Marchesini”, Università degli Studi di Milano, Via Venezian 21, 20133, Milano, Italy

\section{SUPPORTING INFORMATION}

Materials and methods

General procedure for the synthesis of:

- O-allyl carbamates $\mathbf{1 a - d , ~ f - k ~}$

S-4

- O-allyl carbamate 1e

- $\quad N$-allyl ureas 4a-e S-8

- $\quad$-tosyl ortho-allyl anilines 7a,b S-10

- $\quad N$-tosyl glycine derivatives $\mathbf{9 a , b}$ S-12

- $\quad N$-[2-(allyloxy)phenyl]-4-methylbenzenesulfonamide $\mathbf{1 1}$ S-13

General procedure for the synthesis of $N$-tosyl ortho-allyl benzamides 13a-c 
Table S1. Screening of the reaction conditions for the

oxidative aminoazidation reaction: synthesis of $\mathbf{2 a}$

General procedure for the palladium catalyzed aminoazidation reaction:

synthesis of compounds 2a-i; 3h; 5a-e; 6c, 8a,b; 10a,b; 12b; 14a-c; $15 c$

Preparation of 4-(1-azidoethyl)-3-tosyloxazolidin-2-one 16

X-ray crystallography: Experimentals

S-26

Single-crystal X-ray diffraction analysis of $\mathbf{2 h}$

S-26

Single-crystal X-ray diffraction analysis of $\mathbf{5} \mathbf{e}$

S-28

${ }^{1} \mathrm{H}$ and ${ }^{13} \mathrm{C}$ NMR Spectra

S-31 


\section{Materials and methods}

All available chemicals and solvents were purchased from commercial sources and were used without any further purification. Thin layer chromatography (TLC) was performed using $0.25 \mathrm{~mm}$ silica gel precoated plates Si 6o-F254 (Merck, Darmstadt, Germany) visualized by UV-254 light and CAM staining. Purification by flash column chromatography (FCC) was conducted by using silica gel Si 6o, 230-400 mesh, 0.040-0.063 mm (Merck). Melting points were determined on a Stuart Scientific $\mathrm{SMP}_{3}$ and are corrected. ${ }^{1} \mathrm{H}$ and ${ }^{13} \mathrm{C}$ NMR spectra were recorded on a Bruker Avance 400 (400 and $101 \mathrm{MHz}$, respectively) or Bruker Avance 300 (300 and $75 \mathrm{MHz}$, respectively); chemical shifts are indicated in parts per million downfield from $\mathrm{SiMe}_{4}$, using the residual proton $\left(\mathrm{CHCl}_{3}=7.26 \mathrm{ppm}\right)$ and carbon $\left(\mathrm{CDCl}_{3}=77.0 \mathrm{ppm}\right)$ solvent resonances as internal reference. Protons and carbon assignments were achieved by ${ }^{13} \mathrm{C}$-APT, ${ }^{1} \mathrm{H}-{ }^{1} \mathrm{H}$ COSY, and ${ }^{1} \mathrm{H}-{ }^{13} \mathrm{C}$ heteronuclear correlation experiments. Coupling constants values $J$ are given in Hz. Optical rotations were measured on a Perkin-Elmer 241 Polarimeter at $589 \mathrm{~nm}$, using a $10 \mathrm{~cm} \mathrm{x} 5 \mathrm{ml}$ cell and c is in $\mathrm{g} / 100 \mathrm{ml}$. FTIR spectra were recorded on a Tensor 27 (ATR Diamond) Bruker infrared spectrophotometer and are reported in frequency of absorption $\left(\mathrm{cm}^{-1}\right)$. 


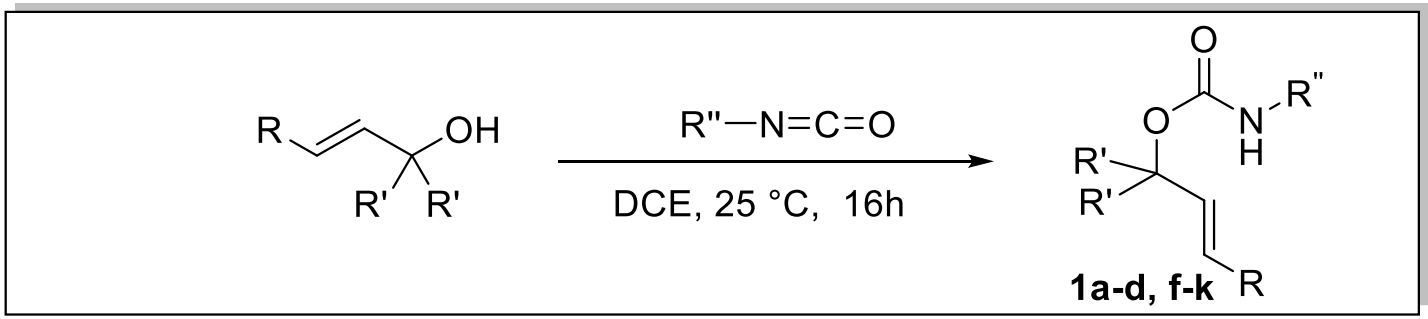

In a round bottom flask was poured the appropriate allyl alcohol (10.0 mmol) dissolved in DCE (15 mL) followed by the addition of the suitable isocyanate (10.0 mmol). The resulting solution was allowed to reach at $25{ }^{\circ} \mathrm{C}$ under $\mathrm{N}_{2}$, and the stirring was continued for further $16 \mathrm{~h}$. Then, after evaporation of the solvent and the volatiles under vacuum (RV), the title compound 1a-d, f-k was obtained. Starting allyl amine and isocyanate, yield, spectroscopic and analytical data of $O$-allyl-carbamates $\mathbf{1 a - d , ~} \mathbf{f - k}$ are as follows.

\section{O-Allyl-N-tosylcarbamate (1a)}

$\sum_{1 a}^{O}$

allyl alcohol (66o $\mu \mathrm{L})$; tosyl isocyanate $(1.98 \mathrm{~g})$. 1a $(2.54 \mathrm{~g}, 99 \%)$. ${ }^{1} \mathrm{H}$ NMR $\left(\mathrm{CDCl}_{3}, 400 \mathrm{MHz}\right) \delta 7.92(\mathrm{~d}, 2 \mathrm{H}, J=8.3 \mathrm{~Hz}), 7.47(\mathrm{~s}, 1 \mathrm{H}), 7.34(\mathrm{~d}, 2 \mathrm{H}, J=8.0 \mathrm{~Hz})$, 5.92-5.69 (m, $1 \mathrm{H}), 5.36-5.12(\mathrm{~m}, 2 \mathrm{H}), 4.67-4.42(\mathrm{~m}, 2 \mathrm{H}), 2.45(\mathrm{~s}, 3 \mathrm{H})$. The characterization of product $\mathbf{1 a}$ is consistent with that reported in the literature. $^{[1]}$

\section{O-Allyl-N-(4-nitrophenyl)carbamate (1b)}<smiles>C=CCOC(=O)Nc1ccc([N+](=O)[O-])cc1</smiles>

allyl alcohol (66o $\mu \mathrm{L})$; 4-nitrophenylisocyanate (1.64 g). 1b (2.09 g, 94\%). ${ }^{1} \mathrm{H}$ NMR $\left(\mathrm{CDCl}_{3}, 400 \mathrm{MHz}\right) \delta 8.22(\mathrm{~d}, 2 \mathrm{H}, J=9.6 \mathrm{~Hz}), 7.58(\mathrm{~d}$, $2 \mathrm{H}, J=8.9 \mathrm{~Hz}), 7.10(\mathrm{br}, 1 \mathrm{H}), 6.04-5.92(\mathrm{~m}, 1 \mathrm{H}), 5.46-5.29(\mathrm{~m}, 2 \mathrm{H})$, $4.75-4.66(\mathrm{~m}, 2 \mathrm{H})$. The characterization of product $\mathbf{1 b}$ is consistent with that reported in the literature. ${ }^{[2]}$

\section{O-Allyl-N-(4-chlorophenyl)carbamate (1c)}<smiles>C=CCOC(=O)Nc1ccc(Cl)cc1</smiles>

allyl alcohol (66o $\mu \mathrm{L})$; 4-chlorophenylisocyanate (1.53 g). 1c (1.88 g, 89\%). ${ }^{1} \mathrm{H} \mathrm{NMR}\left(\mathrm{CDCl}_{3}, 400 \mathrm{MHz}\right) \delta 7.33(\mathrm{~d}, 2 \mathrm{H}, J=8.4 \mathrm{~Hz}), 7.26(\mathrm{dt}, 2 \mathrm{H}, J=5.6$, $2.4 \mathrm{~Hz}), 6.74(\mathrm{~s}, 1 \mathrm{H}), 5.94(\mathrm{ddt}, 1 \mathrm{H}, J=17.0,10.1,5.9 \mathrm{~Hz}), 5.38-5.33(\mathrm{~m}$,

[1] Nicolai, S.; Piemontesi, C.; Waser, J. Angew. Chem. Int. Ed. 2011, 50, 4680

[2] Ren, Y.; Rousseaux, S.A.L. J. Org. Chem. 2018, 82, 913 
$\left.{ }_{1 \mathrm{H}}\right), 5.27-5.23(\mathrm{~m}, 1 \mathrm{H}), 4.63(\mathrm{dt}, 2 \mathrm{H}, J=5.6,1.2 \mathrm{~Hz})$. The characterization of product $1 \mathrm{c}$ is consistent with that reported in the literature. ${ }^{[3]}$

\section{O-Allyl- $N$-(4-methylphenyl)carbamate (1d)}<smiles>C=CCOC(=O)Nc1ccc(C)cc1</smiles>

allyl alcohol (66o $\mu \mathrm{L})$; 4-methylphenylisocyanate (1.33 g). 1d (1.82 g, 95\%). ${ }^{1} \mathrm{H}$ NMR $\left(\mathrm{CDCl}_{3}, 400 \mathrm{MHz}\right) \delta 7.19(\mathrm{~d}, 2 \mathrm{H}, J=7.1 \mathrm{~Hz}), 7.02(\mathrm{~d}, 2 \mathrm{H}, J$ $=8.0 \mathrm{~Hz}), 6.54(\mathrm{~s}, 1 \mathrm{H}), 5.90(\mathrm{ddt}, 1 \mathrm{H}, J=16.4,11.0,4.2 \mathrm{~Hz}), 5.28-5.24(\mathrm{~m}$, $1 \mathrm{H}), 5.20-5.15(\mathrm{~m}, 1 \mathrm{H}), 4.56(\mathrm{dt}, 2 \mathrm{H}, J=5.2,1.1 \mathrm{~Hz}), 2.23(\mathrm{~s}, 3 \mathrm{H})$. The characterization of product $\mathbf{1 d}$ is consistent with that reported in the literature. ${ }^{[3]}$

\section{O-But-3-en-2-yl-N-tosylcarbamate (1f)}<smiles>[3H]NC(=O)OC(C)C=C</smiles>

but-3-en-2-ol (721 mg); tosyl isocyanate (1.98 g). If ( $1.45 \mathrm{~g}, 54 \%) .{ }^{1} \mathrm{H}$ NMR $\left(\mathrm{CDCl}_{3}, 400 \mathrm{MHz}\right): \delta$ 7.93-7.91 (m, 2H), 7.51 (br, $\left.{ }_{1} \mathrm{H}\right), 7.335^{-7.33}(\mathrm{~m}, 2 \mathrm{H})$, $5.73(\mathrm{dd}, 1 \mathrm{H}, J=17.4,6.0 \mathrm{~Hz}), 5.25-5.10\left(\mathrm{~m},{ }_{3} \mathrm{H}\right), 2.45\left(\mathrm{~s},{ }_{3} \mathrm{H}\right), 1.27\left(\mathrm{~d},{ }_{3} \mathrm{H}, J=\right.$ $6.5 \mathrm{~Hz}$ ). The characterization of product $\mathbf{1 f}$ is consistent with that reported in the literature. ${ }^{[1]}$

\section{O-Pent-1-en-3-yl- $N$-tosylcarbamate (1g)}<smiles>[3H]NC(=O)OC(C=C)CC</smiles>

1-penten-3-ol (861 mg); tosyl isocyanate (1.98 g). $1 \mathrm{~g}$ ( $2.69 \mathrm{~g}, 95 \%) .{ }^{1} \mathrm{H}$ NMR $\left(\mathrm{CDCl}_{3}, 400 \mathrm{MHz}\right) \delta 7.91(\mathrm{~d}, 2 \mathrm{H}, J=8.0 \mathrm{~Hz}), 7.47(\mathrm{br}, 1 \mathrm{H}), 7.34(\mathrm{~d}, 2 \mathrm{H}, J=8.0$ Hz), $5.64(\mathrm{ddd}, 1 \mathrm{H}, J=17.1,10.0,16.6 \mathrm{~Hz}), 5.18-5.13(\mathrm{~m}, 2 \mathrm{H}), 5.05-5.00(\mathrm{~m}$, characterization of product $\mathbf{1 g}$ is consistent with that reported in the literature. ${ }^{[4]}$

\section{O-Hex-1-en-3-yl- $N$-tosylcarbamate (1h)}<smiles>C=CC(CCC)OC(=O)N[I-]</smiles>

1-hexen-3-ol (1.00 g); tosyl isocyanate (1.98 g). 1h ( $2.91,98 \%$ yield). ${ }^{1} \mathrm{H}$ $\operatorname{NMR}\left(\mathrm{CDCl}_{3}, 400 \mathrm{MHz}\right) \delta 8.06(\mathrm{~s}, 1 \mathrm{H}), 7.88(\mathrm{~d}, 2 \mathrm{H}, J=8.4 \mathrm{~Hz}), 7.32(\mathrm{~d}, J=$ 8.o Hz, 2H), 5.67-5.60 (m, 1H), 5.24-5.03 (m, 3H), $2.43(\mathrm{~s}, 3 \mathrm{H}), 1.68-1.35$ $(\mathrm{m}, 2 \mathrm{H}), 1.27-1.10(\mathrm{~m}, 2 \mathrm{H}), 0.82(\mathrm{t}, 3 \mathrm{H}, J=7.3 \mathrm{~Hz})$. The characterization of product $\mathbf{l h}$ is consistent with that reported in the literature. ${ }^{[5]}$ 


\section{O-2-Methylbut-3-en-2-yl- $N$-tosylcarbamate (1i)}<smiles>C=CC(C)(C)OC(=O)NC(F)(F)F</smiles>

2-methylbut-3-en-2-ol (861 mg); tosyl isocyanate (1.98 g). 1i ( $2.69 \mathrm{~g}, 95 \%)$. ${ }^{1} \mathrm{H}$ NMR $\left(\mathrm{CDCl}_{3}, 400 \mathrm{MHz}\right) \delta 7.88(\mathrm{~d}, 2 \mathrm{H}, J=8.3 \mathrm{~Hz}), 7.34(\mathrm{~d}, 2 \mathrm{H}, J=8.3$ $\mathrm{Hz}), 5.94(\mathrm{dd}, 1 \mathrm{H}, J=17.3,10.8 \mathrm{~Hz}), 5.13(\mathrm{~d}, 1 \mathrm{H}, J=17.5 \mathrm{~Hz}), 5.04(\mathrm{~d}, 1 \mathrm{H}, J=$ $10.9 \mathrm{~Hz}), 2.43(\mathrm{~s}, 3 \mathrm{H}), 1.44(\mathrm{~s}, 6 \mathrm{H})$. The characterization of product $\mathbf{1 i}$ is consistent with that reported in the literature. ${ }^{[4]}$

\section{O-But-2-en-1-yl- $N$-tosylcarbamate (1j)}<smiles>[3H]NC(=O)OC/C=C/C</smiles>

crotyl alcohol (721 mg); tosyl isocyanate (1.98 g). 1 j (2.56 g, 95\%). ${ }^{1} \mathrm{H}$ NMR $\left(\mathrm{CDCl}_{3}, 400 \mathrm{MHz}\right) \delta 7.95(\mathrm{~d}, 2 \mathrm{H}, J=8.5), 7.45\left(\mathrm{br},{ }_{1} \mathrm{H}\right), 7.36(\mathrm{~d}, 2 \mathrm{H}, J=8.5), 5.87-$ $5.72(\mathrm{~m}, 1 \mathrm{H}), 5.56-5.43(\mathrm{~m}, 2 \mathrm{H}), 4.50(\mathrm{dd}, 2 \mathrm{H}, J=6.7,1.0), 2.47(\mathrm{~s}, 3 \mathrm{H}), 1.68(\mathrm{~d}$, 1j $3 \mathrm{H}, J=6.7)$. The characterization of product $\mathbf{1} \mathbf{j}$ is consistent with that reported in the literature. ${ }^{[4]}$

\section{O-But-2-en-1-yl- $N$-tosylcarbamate (1k)}<smiles>O=C(N[I-])OC/C=C/c1ccccc1</smiles>

cinnamyl alcohol (1.35 g); tosyl isocyanate (1.98 g). 1k (1.33 g, 40\%). ${ }^{1} \mathrm{H}$ NMR $\left(\mathrm{CDCl}_{3}, 400 \mathrm{MHz}\right) \delta$ 7.69-7.6o $(\mathrm{m}, 2 \mathrm{H}), 7.45-7.36(\mathrm{~m}, 2 \mathrm{H}), 7.36-7.25(\mathrm{~m}$, 2H), 7.27-7.25 (m, $1 \mathrm{H})$, 7.22-7.17 (m, 2H), 6.55-6.44 (m, $2 \mathrm{H}), 6.25\left(\mathrm{dt},{ }_{1} \mathrm{H}, J=\right.$

$1 \mathrm{k}$ 15.9, $5.7 \mathrm{~Hz}), 4.42(\mathrm{dd}, 2 \mathrm{H}, J=5.8,1.6 \mathrm{~Hz}), 2.32(\mathrm{~s}, 3 \mathrm{H})$. The characterization of product $\mathbf{1 k}$ is consistent with that reported in the literature. ${ }^{[6]}$

[6] Chow, S.; Steven, M.Y.; Odell, L.R. J. Org. Chem. 2016, 81, 2681 


\section{Preparation of $O$-allyl carbamate (1e)}<smiles>C=CCOC(=O)NS(=O)(=O)c1ccccc1[N+](=O)[O-]</smiles>

Triethylamine $(1.50 \mathrm{~mL}$, $11.0 \mathrm{mmol})$ was added to a solution of allylchloroformate (1.33 g, $11 \mathrm{mmol})$, DMAP (122 mg, $1.0 \mathrm{mmol}$ ) and 2nitrobenzenesulfonamide ( $2.02 \mathrm{~g}, 10.00 \mathrm{mmol})$ in dry $\mathrm{CH}_{2} \mathrm{Cl}_{2}(20 \mathrm{~mL})$ at $\mathrm{o}{ }^{\circ} \mathrm{C}$. Then the reaction was allowed to warm to room temperature After $5 \mathrm{~h}$, the reaction was diluted with $\mathrm{CH}_{2} \mathrm{Cl}_{2}(10 \mathrm{ml})$, washed with $\mathrm{HCl} 10 \%$ solution $(2 \times 5 \mathrm{~mL})$, saturated $\mathrm{NaHCO}_{3}$ solution $(2 \times 10 \mathrm{~mL})$ and brine $(10 \mathrm{~mL})$, dried over $\mathrm{MgSO}_{4}$ and filtered. After evaporation of the solvent under vacuum, the residue was purified by FCC$\mathrm{CH}_{2} \mathrm{Cl}_{2} / \mathrm{MeOH}$ (19:1) to afford compound 1 e (2.00 g, 70\%) as a colorless oil. ${ }^{1} \mathrm{H} \mathrm{NMR}\left(\mathrm{CDCl}_{3}\right.$, $400 \mathrm{MHz}) \delta 8.40(\mathrm{~d}, 1 \mathrm{H}, J=7.6 \mathrm{~Hz}), 7.91-7.80(\mathrm{~m}, 4 \mathrm{H}), 5.93-5.83(\mathrm{~m}, 1 \mathrm{H}), 5.35(\mathrm{~d}, 1 \mathrm{H}, J=17.3$ $\mathrm{Hz}), 5.30(\mathrm{~d}, 1 \mathrm{H}, J=10.5 \mathrm{~Hz}), 4.64(\mathrm{~d}, 2 \mathrm{H}, J=5.7 \mathrm{~Hz}) \cdot{ }^{13} \mathrm{C} \mathrm{NMR}\left(\mathrm{CDCl}_{3}, 101 \mathrm{MHz}\right) \delta 149.8$, 148.2, 135.0, 133.5, 132.6, 131.5, 130.6, 125.2, 119.8, 67.8. Anal. Calcd. for $\mathrm{C}_{10} \mathrm{H}_{10} \mathrm{~N}_{2} \mathrm{O}_{6} \mathrm{~S}: \mathrm{C}, 41.96 ; \mathrm{H}$, 3.52; N, 9.79. Found: C, 42.00; H, 3.55; N, 9.76. 


\section{General procedure for the synthesis of $N$-allyl ureas 4 a-e}

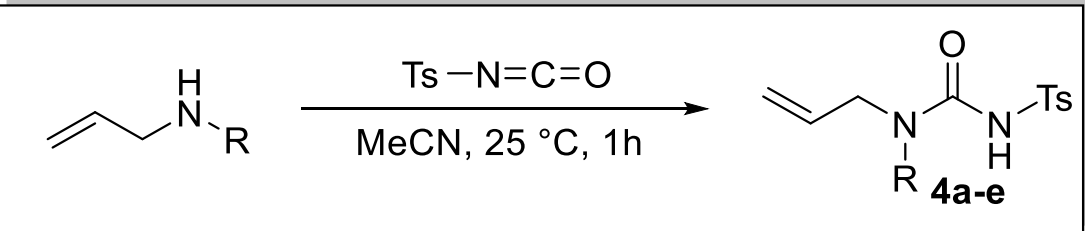

Tosyl isocyanate $(1.98 \mathrm{~g}, 10.0 \mathrm{mmol})$ was added to a solution of the appropriate allyl amine (10.0 mmol) in $\mathrm{MeCN}(15 \mathrm{~mL})$. Then, the solution was stirred at $25{ }^{\circ} \mathrm{C}$ for $1 \mathrm{~h}$ under $\mathrm{N}_{2}$ atmosfere. The volatiles were removed under reduced pressure and the residue was used without any other purification. Starting allyl amine, reaction time, yield and physical, spectroscopic, and analytical data of ureas $\mathbf{4 a - e}$ are as follows.

\section{$N$-Allyl- $N$-methyl- $N$-tosyl-urea (4a)}

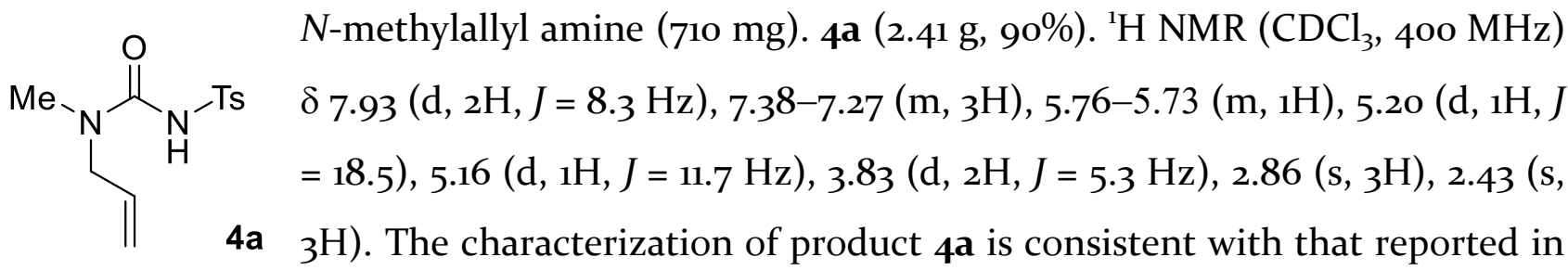
the literature. ${ }^{[5]}$

\section{$N$-Allyl-N-cyclohexyl-N-tosyl-urea (4b)}<smiles>C=CCN(C(=O)N[AsH3])C1CCCCC1</smiles>

$\mathrm{N}$-allylcyclohexyl amine (1.39 g). 4b (1.04 g, 75\%); orange wax. ${ }^{1} \mathrm{H}$ NMR $\left(\mathrm{CDCl}_{3}, 400 \mathrm{MHz}\right) \delta$ 7.94-7.92 (m, 2H), 7.34-7.32 (m, 2H), 7.26-7.24 (m, $1 \mathrm{H})$, 5.84-5.77 (m, $1 \mathrm{H})$, 5.28-5.21 (m, $2 \mathrm{H})$, 4.07-4.04 (m, $1 \mathrm{H}), 3.81-3.79(\mathrm{~m}$,

4b $2 \mathrm{H}), 2.44(\mathrm{~s}, 3 \mathrm{H}), 1.74-1.63(\mathrm{~m}, 5 \mathrm{H}), 1.28-1.04(\mathrm{~m}, 5 \mathrm{H}) .{ }^{13} \mathrm{C}$ NMR $\left(\mathrm{CDCl}_{3}, 101\right.$ MHz) $\delta 151.6,144.4,136.5,134.6,129.5$ (2 C), 128.3 (2 C), 117.3, 54.6, 44.7, $30.6\left(2 \mathrm{CH}_{2}\right), 25.5$ (2 $\left.\mathrm{CH}_{2}\right), 25.3,21.6$; IR $v_{\max } 3387,3253,1701,1429,1360,1163 \mathrm{~cm}^{-1}$. Anal. Calcd. for $\mathrm{C}_{17} \mathrm{H}_{24} \mathrm{~N}_{2} \mathrm{O}_{3} \mathrm{~S}: \mathrm{C}$, 6o.69; H, 7.19; N, 8.33. Found: C, 60.90; H, 7.38; N, 8.17.

\section{$N$-Allyl- $N$-phenyl- $N$-tosyl-urea (4c)}<smiles>C=CCN(C(=O)N[As])c1ccccc1</smiles>

$N$-allylaniline (1.33 g). 4c (1.31 g, 98\%). ${ }^{1} \mathrm{H} \mathrm{NMR}\left(\mathrm{CDCl}_{3}, 400 \mathrm{MHz}\right) \delta 7.93$ $(\mathrm{d}, 2 \mathrm{H}, J=8.3 \mathrm{~Hz}), 7.53-7.38(\mathrm{~m}, 3 \mathrm{H}), 7.38-7.26(\mathrm{~m}, 2 \mathrm{H}), 7.19(\mathrm{~d}, 2 \mathrm{H}, J=7.2$ Hz), $7.09(\mathrm{br}, 1 \mathrm{H}), 5.81-5.78(\mathrm{~m}, 1 \mathrm{H}), 5.13-4.97(\mathrm{~m}, 2 \mathrm{H}), 4.18(\mathrm{~d}, 2 \mathrm{H}, J=6.3$ 4c $\mathrm{Hz}), 2.46(\mathrm{~s}, 3 \mathrm{H})$. The characterization of product $4 \mathrm{c}$ is consistent with that reported in the literature. ${ }^{[5]}$ 
<smiles>[M]C(c1ccccc1)N(CC=C)C(=O)N[As]</smiles>

$(R)-(+)-N$-allyl- $\alpha$-methyl-benzylamine (1.61 g). 4d (1.56 g, 97\%); orange wax; $[\alpha]_{\mathrm{D}}^{20}=+91.66\left(\mathrm{c} 1, \mathrm{CH}_{2} \mathrm{Cl}_{2}\right) .{ }^{1} \mathrm{H} \mathrm{NMR}\left(\mathrm{CDCl}_{3}, 400 \mathrm{MHz}\right) \delta 7.91(\mathrm{~d}$, $2 \mathrm{H}, J=7.7 \mathrm{~Hz}), 7.34-7.26(\mathrm{~m}, 8 \mathrm{H}), 5.62-5.58(\mathrm{~m}, 2 \mathrm{H}), 5.28-5.21(\mathrm{~m}, 2 \mathrm{H})$,

4d $3.64-3.54(\mathrm{~m}, 2 \mathrm{H}), 2.45(\mathrm{~s}, 3 \mathrm{H}), 1.46(\mathrm{~d}, 3 \mathrm{H}, J=6.8 \mathrm{~Hz}) .{ }^{13} \mathrm{C} \mathrm{NMR}\left(\mathrm{CDCl}_{3}\right.$, $101 \mathrm{MHz}) \delta 152.1,144.4,139.8,136.5,134.5,129.5(2 \mathrm{C}), 128.7(2 \mathrm{C}), 128.4(2 \mathrm{C}), 127.9,127.3(2 \mathrm{C})$, 118.5, 52.6, 45.9, 21.7, 16.6; IR $v_{\max }$ 3377, 3249, 1698, 1422, 1361, $1145 \mathrm{~cm}^{-1}$. Anal. Calcd. for $\mathrm{C}_{19} \mathrm{H}_{22} \mathrm{~N}_{2} \mathrm{O}_{3} \mathrm{~S}: \mathrm{C}, 63.66 ; \mathrm{H}, 6.19 ; \mathrm{N}, 7.82$. Found: C, 63.97; H, 6.46; N, 7.58.

\section{$N$-(Allyl(benzyl)carbamoyl)-4-methylbenzenesulfonamide (4e)}

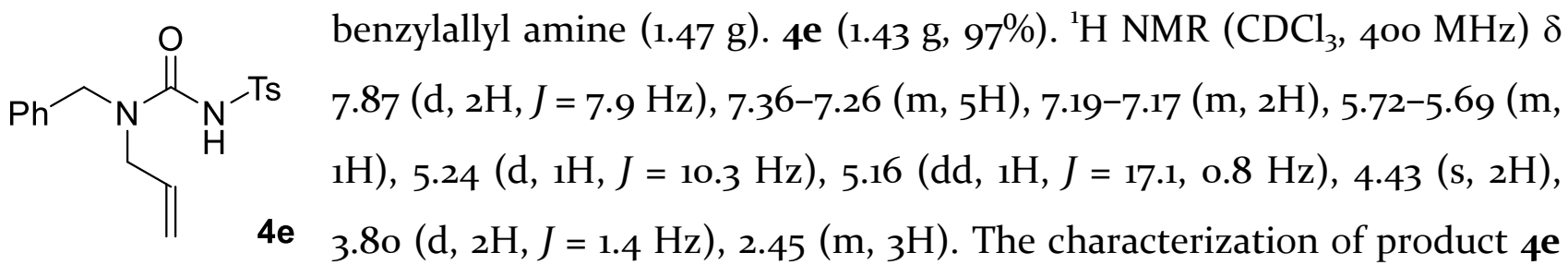
is consistent with that reported in the literature. ${ }^{[7]}$ 
General procedure for the synthesis of $N$-tosyl ortho-allyl anilines $7 \mathbf{a}, \mathbf{b}^{[8]}$

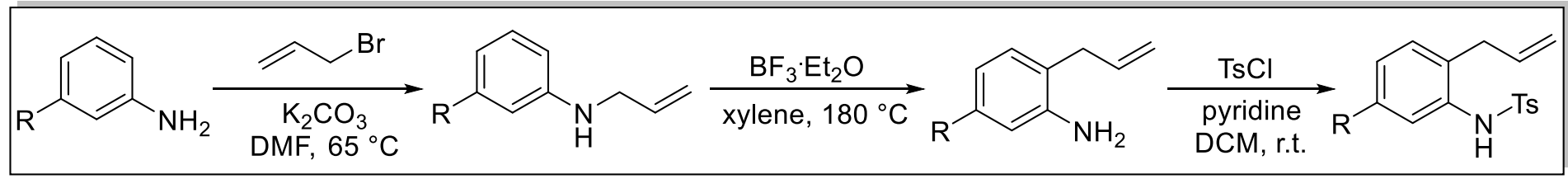

In a round bottom flask, a heterogeneous mixture of the appropriate aniline (10.0 mmol), anhydrous $\mathrm{K}_{2} \mathrm{CO}_{3}(1.38 \mathrm{~g}, 10.0 \mathrm{mmol})$ and allyl bromide $(1.33 \mathrm{~g}$, $11.0 \mathrm{mmol})$ in anhydrous DMF $(20 \mathrm{~mL})$, was stirred at reflux for 16 hours. After cooling, the crude product was diluted with $\mathrm{CH}_{2} \mathrm{Cl}_{2}(10 \mathrm{~mL})$ and filtered through a celite pad. The solvent was evaporated under reduced pressure and the residue was purified by FCC-AcOEt/hexane (4:6). The isolated $N$ allylaniline derivative was dissolved in xylene (10 $\mathrm{mL})$ and put in a screw cap vial. Then, $\mathrm{BF}_{3} \cdot \mathrm{Et}_{2} \mathrm{O}$ (10.0 mmol) was added dropwise and the resulting solution was stirred at $25^{\circ} \mathrm{C}$ for 10 minutes and, subsequently, warmed to $180{ }^{\circ} \mathrm{C}$ for $4 \mathrm{~h}$. After cooling, the reaction was quenched by $\mathrm{NaOH}[2 \mathrm{~N}]$. The mixture was poured into a separatory funnel and extracted with $\mathrm{Et}_{2} \mathrm{O}(1 \mathrm{x} 30 \mathrm{~mL})$, the organic phase was washed with $\mathrm{H}_{2} \mathrm{O}(1 \times 20 \mathrm{~mL})$ and once with brine $(20 \mathrm{~mL})$, dried over $\mathrm{MgSO}_{4}$ and the solvent evaporated under reduced pressure to afford the target substituted 2-allylaniline after purification by FCC-AcOEt/hexane (4:6). This latter compound was dissolved in dry $\mathrm{CH}_{2} \mathrm{Cl}_{2}(20 \mathrm{~mL})$; pyridine (10.0 mmol) and TsCl ( $2.10 \mathrm{~g}$, $11.0 \mathrm{mmol})$ were added dropwise in sequence at $\mathrm{o}{ }^{\circ} \mathrm{C}$. Then the reaction was allowed to warm to room temperature. After $30 \mathrm{~h}$, the reaction was diluted with $\mathrm{CH}_{2} \mathrm{Cl}_{2}(10 \mathrm{ml})$, washed with $\mathrm{HCl} 10 \%$ solution $(2 \times 5 \mathrm{~mL})$, saturated $\mathrm{NaHCO}_{3}$ solution $(2 \times 10 \mathrm{~mL})$ and brine (10 $\mathrm{mL}$ ), dried over $\mathrm{MgSO}_{4}$ and filtered. After evaporation of the solvent under vacuum, the residue was purified by FCC-AcOEt/hexane (4:6). Starting aniline, yield, spectroscopic and analytical data of compounds $\mathbf{7 a , b}$ are as follows.

\section{2-Allyl-N-tosylaniline (7a)}

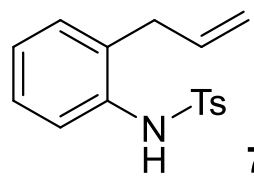

aniline (931 mg). 7a (948 mg, 33\%). ${ }^{1} \mathrm{H} \mathrm{NMR}\left(\mathrm{CDCl}_{3}, 400 \mathrm{MHz}\right) \delta 7.59(\mathrm{~d}$, $2 \mathrm{H}, J=8.3 \mathrm{~Hz}), 7.37(\mathrm{~d}, 2 \mathrm{H}, J=8.4 \mathrm{~Hz}), 7.28-7.01(\mathrm{~m}, 4 \mathrm{H}), 6.50(\mathrm{bs}, 1 \mathrm{H})$,

7a $5.78(\mathrm{dt}, 1 \mathrm{H}, J=12.0,6.0 \mathrm{~Hz}), 5.03(\mathrm{dd}, 2 \mathrm{H}, J=18.8,1.5 \mathrm{~Hz}), 3.01(\mathrm{~d}, 2 \mathrm{H}, J=$

[8] Muniz, K.; Lishchynskyi, A.; Streuff, J.; Nieger, M.; Escudero-Adan, E. C.; Belmonte, M. Chem. Commun., 2011, 47,4911 
$5.9 \mathrm{~Hz}), 2.39(\mathrm{~s}, 3 \mathrm{H})$. The characterization of product $7 \mathrm{a}$ is consistent with that reported in the literature. ${ }^{[9]}$

\section{2-Allyl-N-tosylaniline (7b)}

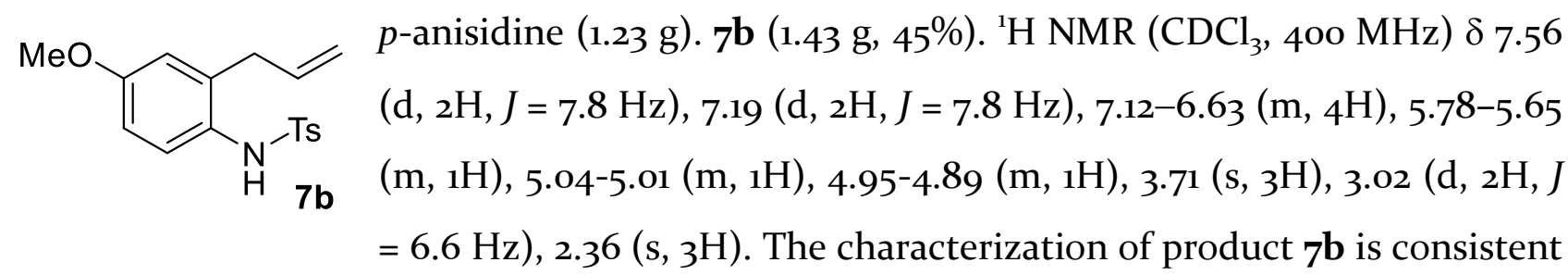
with that reported in the literature. ${ }^{[10]}$ 


\section{General procedure for the synthesis of $N$-tosyl glycine derivatives 9a,b}

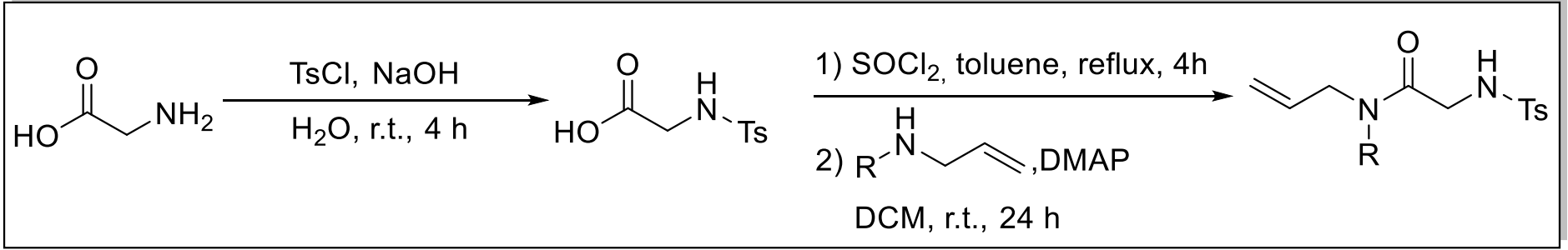

To a solution of glycine hydrochloride $(1.7 \mathrm{~g}, 10 \mathrm{mmol})$ in $\mathrm{H}_{2} \mathrm{O}[2 \mathrm{M}], \mathrm{NaOH}[2 \mathrm{M}$ ] was added until $\mathrm{pH}$ 9. Then $\mathrm{TsCl}(1.9 \mathrm{~g}, 10 \mathrm{mmol})$ was added and the resulting solution was stirred at 25 ${ }^{\circ} \mathrm{C}$ for 4 hours. The solid was filtered off, dissolved in $\mathrm{PhCH}_{3}$ and $\mathrm{SOCl}_{2}$ (3.57 g, $30.0 \mathrm{mmol}$ ) was added. The reaction was stirred for $4 \mathrm{~h}$ at reflux. After cooling and evaporation of the solvent under vacuum, the residue was suspended in $\mathrm{CH}_{2} \mathrm{Cl}_{2}\left[0.5 \mathrm{M}\right.$ ]; then, $\mathrm{Et}_{3} \mathrm{~N}$ (1.01 g, 10.0 $\mathrm{mmol})$ and the appropriate $N$-allylamine $(1.2 \mathrm{mmol})$ were added ad $\mathrm{o}{ }^{\circ} \mathrm{C}$. The resulting solution was allowed to reach at room temperature, and the stirring was continued for further $16 \mathrm{~h}$. The reaction was washed with $\mathrm{HCl} 5 \%$ solution $(5 \mathrm{~mL})$, brine (10 $\mathrm{mL})$, saturated $\mathrm{NaHCO}_{3}$ solution $(5 \mathrm{~mL})$ and brine $(10 \mathrm{~mL})$, dried over $\mathrm{MgSO}_{4}$ and filtered. After evaporation of the solvent under vacuum, the crude was purified by FCC-AcOEt/hexane (4:6) affording the target compound. Starting aniline, yield, spectroscopic and analytical data of compounds $\mathbf{9 a} \mathbf{a} \mathbf{b}$ are as follows.

\section{$N$-Allyl-N-cyclohexyl-2-[(4-methylphenyl)sulfonamido]acetamide (9a)}<smiles>C=CCN(C)C(=O)CNS</smiles>

$N$-cyclohesyl- $N$-allylamine (1.53 g). 9a (1.16 g, 76\%). Major rotamer: ${ }^{1} \mathrm{H}$ $\operatorname{NMR}\left(\mathrm{CDCl}_{3}, 300 \mathrm{MHz}\right) \delta 8.30(\mathrm{~d}, 2 \mathrm{H}, J=8.8 \mathrm{~Hz}), 8.03(\mathrm{~d}, 2 \mathrm{H}, J=8.8$ 9a Hz), $6.14(\mathrm{br}, 1 \mathrm{H})$, 5.61-5.70 (m, $1 \mathrm{H}), 4.97-5.17(\mathrm{~m}, 2 \mathrm{H}), 4.18-4.25(\mathrm{~m}$, $\left.{ }_{1 H}\right), 3.71-3.95(\mathrm{~m}, 4 \mathrm{H}), 0.98-1.80(\mathrm{~m}, 10 \mathrm{H})$. The characterization of product 9a is consistent with that reported in the literature. ${ }^{[1]}$

$N$-Allyl- $N$-phenyl-2-[(4-methylphenyl)sulfonamido]acetamide (9b).<smiles>C=CCN(C(=O)CNS)c1ccccc1</smiles>
$N$-phenyl- $N$-allylamine (1.59 g). 9b (910 mg, 57\%). Major rotamer: ${ }^{1} \mathrm{H}$ $\operatorname{NMR}\left(\mathrm{CDCl}_{3}, 300 \mathrm{MHz}\right) \delta 7.65(\mathrm{~d}, 2 \mathrm{H}, J=8.2 \mathrm{~Hz}), 7.38-7.42(\mathrm{~m}, 2 \mathrm{H})$, 9b $\quad 7.25-7.31(\mathrm{~m}, 3 \mathrm{H}), 6.95-7.02(\mathrm{~m}, 2 \mathrm{H}), 5.73(\mathrm{br}, 1 \mathrm{H}), 5.61-5.65\left(\mathrm{~m},{ }_{1} \mathrm{H}\right)$, $5.07(\mathrm{~d}, 1 \mathrm{H}, J=10.2 \mathrm{~Hz}), 4.97(\mathrm{~d}, 1 \mathrm{H}, J=17.0 \mathrm{~Hz}), 4.16(\mathrm{~d}, 2 \mathrm{H}, J=6.4 \mathrm{~Hz}), 3.42(\mathrm{~d}, 2 \mathrm{H}, J=4.8$ 
$\mathrm{Hz}), 2.43\left(\mathrm{~s},{ }_{3} \mathrm{H}\right)$. The characterization of product $\mathbf{9 b}$ is consistent with that reported in the literature. ${ }^{[11]}$

\section{Preparation of $N$-[2-(allyloxy)phenyl]-4-methylbenzenesulfonamide (11)}<smiles>C=CCOc1ccccc1NC(F)(F)F</smiles>

In a screw cap vial, a heterogeneous mixture of aminophenol (2 mmol) solution in dry THF/DMF (5:1, $10 \mathrm{~mL})$, anhydrous $\mathrm{K}_{2} \mathrm{CO}_{3}(2.6$

11 mmol) allylbromide $(2.6 \mathrm{mmol})$, was stirred at $25^{\circ} \mathrm{C}$ until no starting aminophenol was detectable (48 h; TLC control - AcOEt/hexane 4:6). After evaporation of the solvent under vacuum, the residue was diluted with AcOEt, washed with $\mathrm{H}_{2} \mathrm{O}(3 \times 10 \mathrm{~mL})$, dried over $\mathrm{MgSO}_{4}$ and filtered. After evaporation of the solvent under vacuum, the obtained $O$-allylaminophenol was dissolved in $\mathrm{CH}_{2} \mathrm{Cl}_{2}[0.2 \mathrm{M}]$, and then, pyridine (711 mg, $10 \mathrm{mmol})$ and tosyl chloride $(2.6 \mathrm{mmol}, 1.3$ equiv.) were added and stirred for $24 \mathrm{~h}$ at room temperature. The crude was then quenched with cold $\mathrm{HCl} 10 \%(20 \mathrm{~mL})$ and extracted with $\mathrm{CH}_{2} \mathrm{Cl}_{2}(25 \mathrm{~mL})$. The organic phase was washed with $\mathrm{H}_{2} \mathrm{O}(2 \mathrm{O} \mathrm{mL})$, saturated $\mathrm{NaHCO}_{3}$ solution $(10 \mathrm{~mL})$ and brine $(10 \mathrm{~mL})$, dried over $\mathrm{MgSO}_{4}$ and filtered. After evaporation of the solvent under vacuum (RV), the crude was purified by FCC (AcOEt/hexane 1:7) to afford compound 11 (558 mg, 92\%); pink solid, mp 100-102 ${ }^{\circ} \mathrm{C} .{ }^{1} \mathrm{H}$ NMR $\left(\mathrm{CDCl}_{3}, 400 \mathrm{MHz}\right) \delta 7.62(\mathrm{~d}, 2 \mathrm{H}, J=8.2 \mathrm{~Hz}), 7.55\left(\mathrm{dd},{ }_{1} \mathrm{H}, J=7.9,1.7 \mathrm{~Hz}\right)$, 7.06-6.95 (m, $\left.{ }_{4} \mathrm{H}\right), 6.89(\mathrm{td}, 1 \mathrm{H}, J=7.7,1.3 \mathrm{~Hz}), 6.71(\mathrm{dd}, 1 \mathrm{H}, J=8.1,1.3 \mathrm{~Hz}), 5.98-5.64(\mathrm{~m}, 1 \mathrm{H}), 5.35-5.03(\mathrm{~m}$, $2 \mathrm{H}), 4.33(\mathrm{dt}, 2 \mathrm{H}, J=5.4,1.4 \mathrm{~Hz}), 2.34(\mathrm{~s}, 3 \mathrm{H})$. The characterization of product 11 is consistent with that reported in the literature. ${ }^{[12]}$

\footnotetext{
${ }^{12}$ Majumdar, K. C.; Ray, Krishanu; Ponra, Sudipta Tetrahedron Letters 2o1o, 51, 41, 5437-5439 


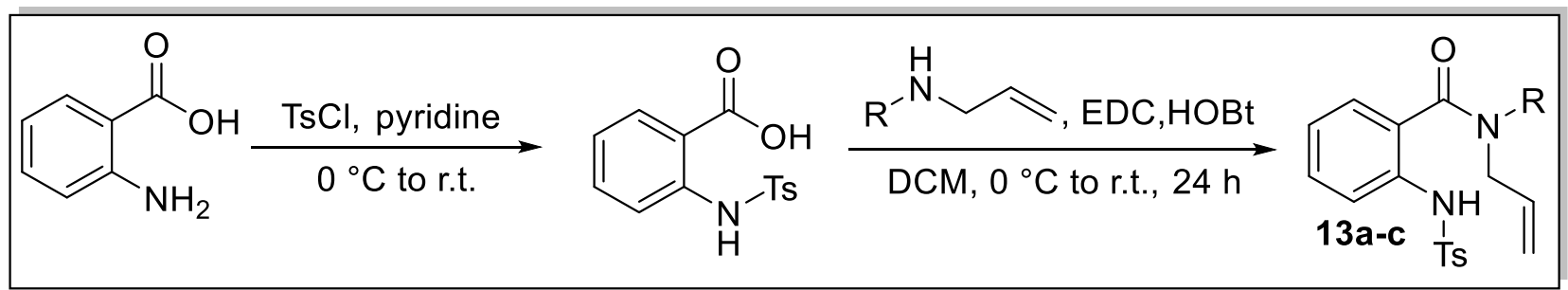

To a solution of anthranilic acid (410 $\mathrm{mg}, 1.42 \mathrm{mmol}$ ) in dry pyridine $(3 \mathrm{~mL})$, tosyl chloride (1.1 equiv.) was added at $\mathrm{o}^{\circ} \mathrm{C}$ under nitrogen atmosphere. After $10 \mathrm{~h}$, the reaction was quenched with ice/water and the aqueous layer was extracted with $\mathrm{CH}_{2} \mathrm{Cl}_{2}$. The organic layers were combined, washed with $\mathrm{HCl} 10 \%$ (5 mL), brine (10 $\mathrm{mL}$ ), dried over $\mathrm{MgSO}_{4}$, filtered and concentrated under reduced pressure. The crude product was purified by crystallization (Hex/EtOAc) to afford the desired $N$-( $p$-tosyl)anthranilic acid derivative (88\% yield). The obtained solid was dissolved in $\mathrm{CH}_{2} \mathrm{Cl}_{2}(4 \mathrm{~mL})$ and $\mathrm{EDC}$ (1.2 equiv) and 1hydroxybenzotriazole ( 1.2 equiv.) were added at $\mathrm{o}^{\circ} \mathrm{C}$. After $1 \mathrm{~h}$, the suitable allyl amine ( 1 equiv.) and $N, N$ '-diisopropylethylamine (1.5 equiv.) were dropped. The reaction mixture was allowed to warm to room temperature and stirred for $30 \mathrm{~h}$. Then the reaction mixture was washed with $5 \% \mathrm{KHSO}_{4}$, saturated $\mathrm{NaHCO}_{3}$ and brine. After drying over $\mathrm{Na}_{2} \mathrm{SO}_{4}$, the solvent was removed under reduced pressure. Purification of the crude by FCC (AcOEt/hexane 1:4) afforded the desired product. Yield, physical, spectroscopic and analytical data of compounds 13a-c are as follows.

\section{$N$-Allyl- $N$-methyl-2-tosylamino-benzamide (13a)}<smiles>C=CCN(C)C(=O)c1ccccc1N(C)C</smiles>

$13 a$

13a (361 mg, 74\%); orange wax. ${ }^{1} \mathrm{H}$ NMR $\left(\mathrm{CDCl}_{3}, 400 \mathrm{MHz}\right) \delta 8.51(\mathrm{~s}, 1 \mathrm{H})$, 7.71-7.64 (m, 3H), 7.43-7.08 (m, 5H), 5.87-5.58 (m, $1 \mathrm{H}), 5.32-5.25(\mathrm{~m}$, $2 \mathrm{H}$ ), $3.98(\mathrm{br}, 1 \mathrm{H}), 3.37$ (br, $1 \mathrm{H}), 2.92(\mathrm{br}, 1.5 \mathrm{H}), 2.48$ (br, $1.5 \mathrm{H}), 2.38$ (s, $3 \mathrm{H})$. The characterization of product $13 \mathrm{a}$ is consistent with that reported in the literature. ${ }^{[13]}$ 


\section{$N$-Allyl- $N$-benzyl-2-tosylamino-benzamide (13b)}<smiles>C=CCN(Cc1ccccc1)C(=O)c1ccccc1N[As]</smiles>

13b (117 mg, 20\%); orange wax. ${ }^{1} \mathrm{H} \mathrm{NMR}\left(\mathrm{CDCl}_{3}, 400 \mathrm{MHz}\right) \delta 8.14(\mathrm{br}$, 1H), $7.64(\mathrm{~d}, 2 \mathrm{H}, J=7.8 \mathrm{~Hz}), 7.54\left(\mathrm{dd},{ }_{1} \mathrm{H}, J=1.6,8.6 \mathrm{~Hz}\right), 7.31-7.19(\mathrm{~m}$, $3 \mathrm{H}), 7.15(\mathrm{~d}, 2 \mathrm{H}, J=8.3 \mathrm{~Hz}), 7.13(\mathrm{dd}, 2 \mathrm{H}, J=1.5,7.4 \mathrm{~Hz}), 7.07-7.02(\mathrm{~m}$, $2 \mathrm{H}), 3.28(\mathrm{~d}, 2 \mathrm{H}, J=6.3 \mathrm{~Hz}), 2.32(\mathrm{~s}, 3 \mathrm{H})$. The characterization of product $\mathbf{1 3} \mathbf{b}$ is consistent with that reported in the literature. ${ }^{[14]}$

\section{$N$-Allyl- $N$-cyclohexyl-2-tosylamino-benzamide (13c)}<smiles>C=CCN(CCCCC)C(=O)c1ccccc1NC(=O)Cl</smiles>

13c (439 mg, 75\%); orange wax. ${ }^{1} \mathrm{H}$ NMR $\left(\mathrm{CDCl}_{3}, 400 \mathrm{MHz}\right) \delta 8.32$ (br, $\left.{ }_{1 \mathrm{H}}\right), 7.70(\mathrm{~d}, 2 \mathrm{H}, J=8.1 \mathrm{~Hz}), 7.64(\mathrm{~d}, 1 \mathrm{H}, J=8.1 \mathrm{~Hz}), 7.33(\mathrm{dd}, 1 \mathrm{H}, J=7.7$, $8.1 \mathrm{~Hz}), 7.24(\mathrm{~d}, 2 \mathrm{H}, J=8.1 \mathrm{~Hz}), 7.17(\mathrm{~d}, 1 \mathrm{H}, J=7.5 \mathrm{~Hz}), 7.06(\mathrm{dd}, 1 \mathrm{H}, J=$

13c $7.7,7.5 \mathrm{~Hz}), 5.79-6.01(\mathrm{~m}, 1 \mathrm{H}), 5.27-5.31(\mathrm{~m}, 2 \mathrm{H}), 3.42-4.11(\mathrm{~m}, 3 \mathrm{H}), 2.38$ $(\mathrm{s}, 3 \mathrm{H}), 1.12-1.84(\mathrm{~m}, 10 \mathrm{H})$. The characterization of product $13 \mathrm{c}$ is consistent with that reported in the literature. ${ }^{[12]}$ 
Table S1. Screening of the reaction conditions for the oxidative aminoazidation reaction ${ }^{\mathrm{a}}$

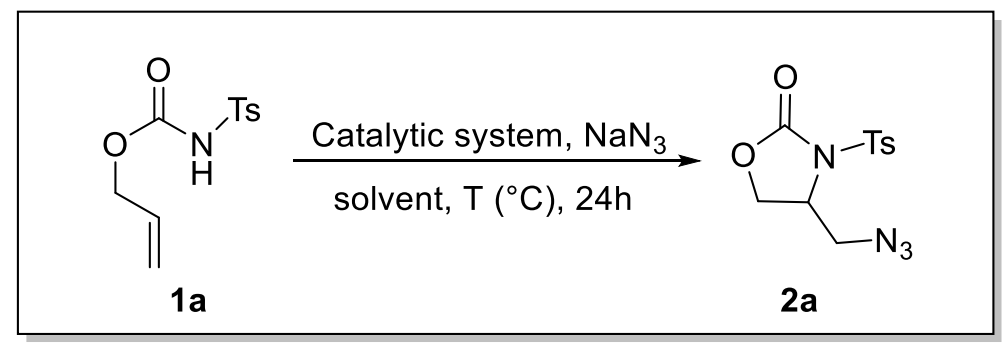

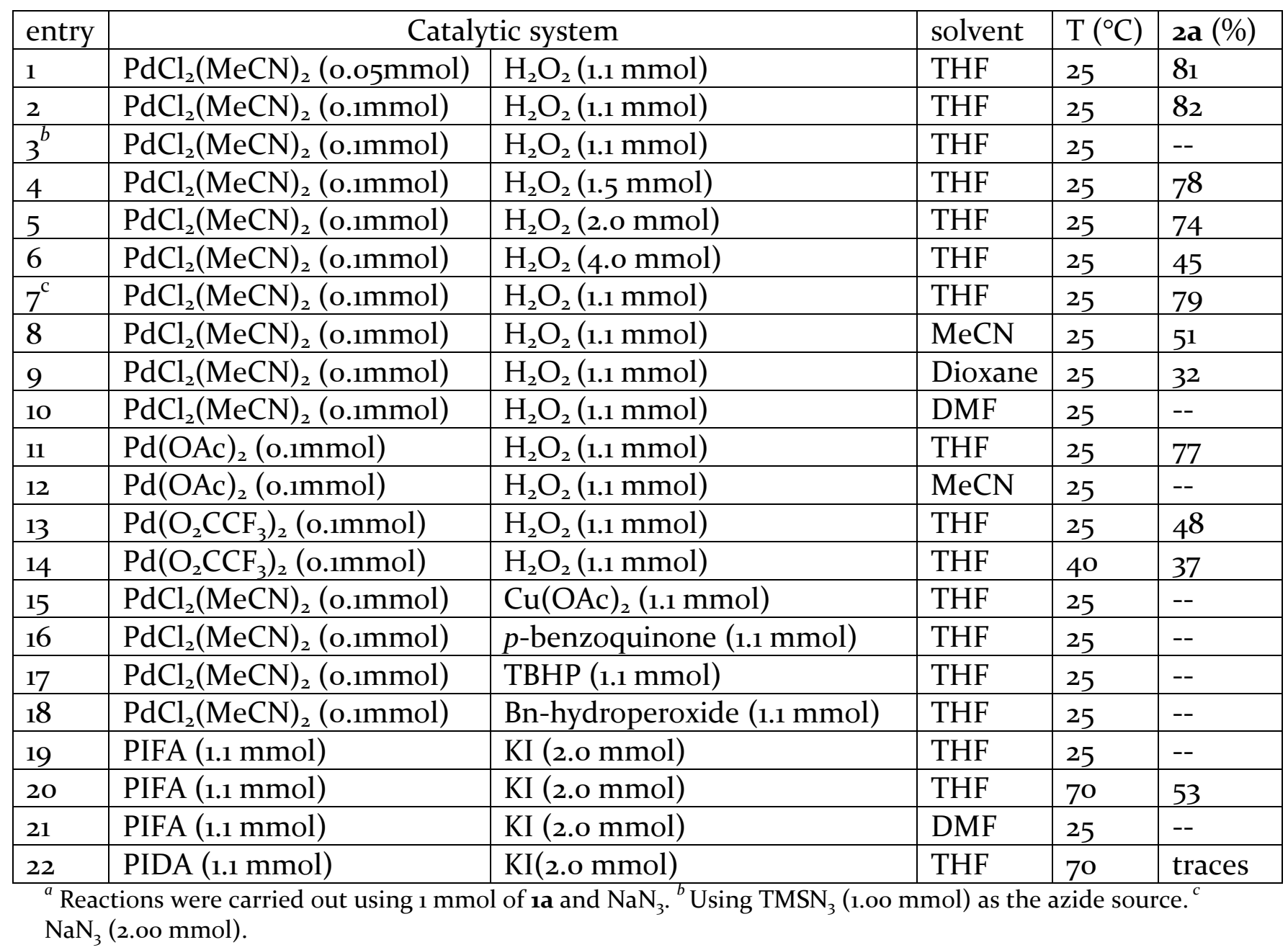




\section{General procedure for the palladium catalyzed aminoazidations:}

synthesis of 2a,b,e-i; 3h; 5a-e; 6c, 8a,b; 10a,b; 12b; 14a-c; 15c

To a solution of starting allyl compound 1, 4, 7, 9, 11, 13 (1.00 $\mathrm{mmol})$ in THF (5 mL) were added in sequence: the Pd-catalyst ( $0.05 \mathrm{mmol}$ ), $\mathrm{NaN}_{3}\left(65 \mathrm{mg}, 1.00 \mathrm{mmol}\right.$ ) and $\mathrm{H}_{2} \mathrm{O}_{2} 30 \%$ aqueous solution $(0.16 \mathrm{~mL}, 1.10 \mathrm{mmol})$. The resulted solution was stirred at room temperature until no starting allyl compound was detectable (TLC analysis). Then, the crude mixture was diluted with AcOEt and filtered through a celite pad. The solvent was evaporated under reduced pressure and the residue was purified by FCC. Starting allyl derivativative, palladium catalyst, reaction time, chromatographic eluant, yield and physical, spectroscopic and analytical data of compound 2a,b,e-i; $3 \mathbf{h} ; \mathbf{5 a - e ; ~ 6 c ; ~ 8 a , b ; ~ 1 0 a , b ; ~ 1 2 b ; ~ 1 4 a - c ; ~}$ 15C are as follows.

\section{4-(Azidomethyl)-3-tosyloxazolidin-2-one (2a)}

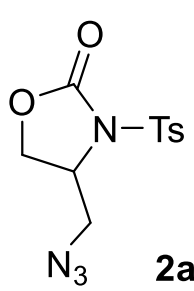

1a (255 mg); $\mathrm{PdCl}_{2}(\mathrm{MeCN})_{2}(13 \mathrm{mg}) ; \mathrm{FCC}-\mathrm{AcOEt} / \mathrm{h}$ exane (4:6). $2 \mathbf{a}(240 \mathrm{mg}, 81 \%$, 24 h); white solid, m.p.: $111-112{ }^{\circ} \mathrm{C} .{ }^{1} \mathrm{H}$ NMR $\left(\mathrm{CDCl}_{3}\right.$, $\left.400 \mathrm{MHz}\right) \delta 7.96$ (d, $2 \mathrm{H}, J=$ $8.2 \mathrm{~Hz}), 7.38(\mathrm{~d}, 2 \mathrm{H}, J=8.1 \mathrm{~Hz}), 4.57-4.51(\mathrm{~m}, 1 \mathrm{H}),, 4.38(\mathrm{t}, 1 \mathrm{H}, J=8.8 \mathrm{~Hz}), 4.21$ $(\mathrm{dd}, 1 \mathrm{H}, J=8.9,3.7 \mathrm{~Hz}), 3.87(\mathrm{dd}, 1 \mathrm{H}, J=12.8,5.7 \mathrm{~Hz}), 3.73(\mathrm{dd}, 1 \mathrm{H}, J=12.8,2.5$ $\mathrm{Hz}), 2.46(\mathrm{~s}, 3 \mathrm{H}) ;{ }^{13} \mathrm{C} \mathrm{NMR}\left(\mathrm{CDCl}_{3}, 101 \mathrm{MHz}\right) \delta 151.8,146.1,134.5,129.9$ (2C), 128.5 (2C), 65.6, 55.3, 52.5, 21.7. IR $v$ max 2112, 1771, 1359, 1189, 1170, $1137 \mathrm{~cm}^{-1}$. Anal. Calcd. for $\mathrm{C}_{11} \mathrm{H}_{12} \mathrm{~N}_{4} \mathrm{O}_{4} \mathrm{~S}: \mathrm{C}, 44.59 ; \mathrm{H}, 4.08 ; \mathrm{N}, 18.91$. Found: $\mathrm{C}, 44.44 ; \mathrm{H}, 3.98 ; \mathrm{N}, 19.00$.

\section{4-(Azidomethyl)-3-(4-nitrophenyl)oxazolidin-2-one (2b)}

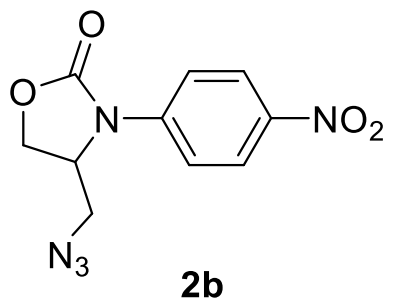

lb (222 mg); $\mathrm{PdCl}_{2}(\mathrm{MeCN})_{2}(13 \mathrm{mg}) ; \mathrm{FCC}-\mathrm{AcOEt} /$ hexane (4:6). 2b (173 mg, 53\%, 6o ${ }^{\circ} \mathrm{C}$ - MW irradiation, $1 \mathrm{~h}$ ); yellow solid, m.p.: 114-115 ${ }^{\circ} \mathrm{C} .{ }^{1} \mathrm{H}$ NMR $\left(\mathrm{CDCl}_{3}, 400 \mathrm{MHz}\right) \delta 8.28(\mathrm{~d}, 2 \mathrm{H}, J=9.3 \mathrm{~Hz}), 7.72(\mathrm{~d}, 2 \mathrm{H}, J$ $=9.3 \mathrm{~Hz}), 4.70-4.66(\mathrm{~m}, \mathrm{iH}), 4.59(\mathrm{t}, \mathrm{1H}, J=8.8 \mathrm{~Hz}), 4.39(\mathrm{dd}, 1 \mathrm{H}, J=$ 9.0, $3.7 \mathrm{~Hz}), 3.70(\mathrm{dd}, 1 \mathrm{H}, J=13.0,5.6 \mathrm{~Hz}), 3.58(\mathrm{dd}, 1 \mathrm{H}, J=13.2,2.9$

$\mathrm{Hz}) ;{ }^{13} \mathrm{C} \mathrm{NMR}\left(\mathrm{CDCl}_{3}, 101 \mathrm{MHz}\right) \delta 154.4,144.2,142.0,125.2(2 \mathrm{C}), 119.9$ (2C), 64.8, 54.8, 50.6; IR $v \max 2108,1748,1594,1500,1288,1197,848 \mathrm{~cm}^{-1}$ Anal. Calcd. For $\mathrm{C}_{10} \mathrm{H}_{9} \mathrm{~N}_{5} \mathrm{O}_{4}$ : C, 45.63; H, 3.45; N, 26.61. Found: C, 45.51; H, 3.36; N, 26.72. 
4-(Azidomethyl)-3-[(2-nitrophenyl)sulfonyl] oxazolidin-2-one (2e)

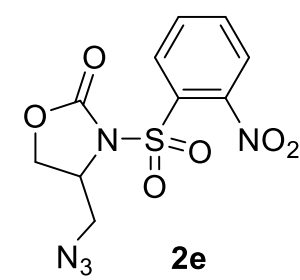

1e (286 mg); $\mathrm{PdCl}_{2}(\mathrm{MeCN})_{2}(13 \mathrm{mg}) ; \mathrm{FCC}-\mathrm{AcOEt} /$ hexane (4:6). 2e (183 mg, $56 \%, 16 \mathrm{~h})$; colourless oil. ${ }^{1} \mathrm{H}$ NMR $\left(\mathrm{CDCl}_{3}, 400 \mathrm{MHz}\right) \delta 8.40(\mathrm{~d}, 1 \mathrm{H}, J=7.5$ $\mathrm{Hz}), 7.79-7.66(\mathrm{~m}, 3 \mathrm{H}), 4.69-4.67(\mathrm{~m}, 1 \mathrm{H}), 4.51(\mathrm{t}, 1 \mathrm{H}, J=8.6 \mathrm{~Hz}), 4.28$ $(\mathrm{dd}, 1 \mathrm{H}, J=8.6,7.2 \mathrm{~Hz}), 3.86(\mathrm{dd}, 1 \mathrm{H}, J=12.8,5.4 \mathrm{~Hz}), 3.67(\mathrm{~d}, 1 \mathrm{H}, J=13.9$ $\mathrm{Hz}) ;{ }^{13} \mathrm{C} \mathrm{NMR}\left(\mathrm{CDCl}_{3}, 101 \mathrm{MHz}\right) \delta 153.4,147.6,135.7,135.3,135.0,132.4,124.6$, 66.5, 56.2, 53.5. Anal. Calcd. For $\mathrm{C}_{10} \mathrm{H}_{9} \mathrm{~N}_{5} \mathrm{O}_{6} \mathrm{~S}: \mathrm{C}, 36.70 ; \mathrm{H}, 2.77 ; \mathrm{N}, 21.40 ;$. Found: C, 37.01; H, 3.09; N, 21.11;.

$\left({ }_{4} S^{*}, 5 S^{*}\right)$-4-(Azidomethyl)-5-methyl-3-tosyloxazolidin-2-one (2f)

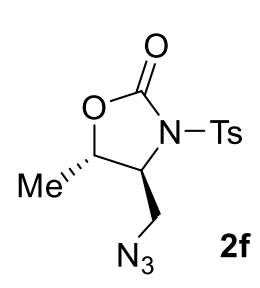
If (269); $\mathrm{PdCl}_{2}(\mathrm{MeCN})_{2}(13 \mathrm{mg}) ; \mathrm{FCC}-\mathrm{CH}_{2} \mathrm{Cl}_{2} /$ hexane (1:9). 2f (189 mg, 61\%, $24 \mathrm{~h})$; yellow wax. ${ }^{1} \mathrm{H}$ NMR $\left(\mathrm{CDCl}_{3}, 400 \mathrm{MHz}\right) \delta 7.95(\mathrm{~d}, 2 \mathrm{H}, J=8.3 \mathrm{~Hz}), 7.38$ $(\mathrm{d}, 2 \mathrm{H}, J=8.1 \mathrm{~Hz}), 4.49-4.44(\mathrm{~m}, 1 \mathrm{H}), 4.03(\mathrm{dd}, 1 \mathrm{H}, J=6.2,3.2 \mathrm{~Hz}), 3.82(\mathrm{dd}$, 2f $1 \mathrm{H}, J=12.7,6.1 \mathrm{~Hz}), 3.74(\mathrm{dd}, 1 \mathrm{H}, J=12.7,2.9 \mathrm{~Hz}), 2.46(\mathrm{~s}, 3 \mathrm{H}), 1.34(\mathrm{~d}, 3 \mathrm{H}, J=$ $6.4 \mathrm{~Hz}) ;{ }^{13} \mathrm{C} \mathrm{NMR}\left(\mathrm{CDCl}_{3}, 101 \mathrm{MHz}\right) \delta 151.3,146.0,134.5,129.9(2 \mathrm{C}), 128.4(2 \mathrm{C}), 74.4,61.7,52.3$, 21.7, 20.7; IR $v_{\max } 2985,2116,1783,1370,1174 \mathrm{~cm}^{-1}$. Anal. Calcd. For $\mathrm{C}_{12} \mathrm{H}_{14} \mathrm{~N}_{4} \mathrm{O}_{4} \mathrm{~S}: \mathrm{C}, 46.45 ; \mathrm{H}$, 4.55; N, 18.05. Found: C, 46.36; H, 4.47; N, 18.15 .

\section{$\left({ }_{4} S^{*}, S^{*}\right)$-4-(Azidomethyl)-5-ethyl-3-tosyloxazolidin-2-one (2g)}<smiles>[Z20]C[C@H]1[C@H](CC)OC(=O)N1[13CH3]</smiles>

$1 \mathrm{~g}(283 \mathrm{mg}) ; \mathrm{PdCl}_{2}(\mathrm{MeCN})_{2}$ (13 mg); FCC-AcOEt/hexane (2:8). 2 g (221 mg, 68\%, $24 \mathrm{~h})$; yellow wax. ${ }^{1} \mathrm{H}$ NMR $\left(\mathrm{CDCl}_{3}, 400 \mathrm{MHz}\right) \delta 7.95(\mathrm{~d}, 2 \mathrm{H}, J=8.3$ $\mathrm{Hz}), 7.37(\mathrm{~d}, 2 \mathrm{H}, J=8.1 \mathrm{~Hz}), 4.29-4.25(\mathrm{~m}, 1 \mathrm{H}), 4.11-4.09(\mathrm{~m}, 1 \mathrm{H}), 3.83(\mathrm{dd}$,

$\left.2 \mathrm{~g} \quad{ }_{1} \mathrm{H}, J=12.6,6.1\right), 3.72(\mathrm{dd}, 1 \mathrm{H}, J=12.6,2.9 \mathrm{~Hz}), 2.46(\mathrm{~s}, 3 \mathrm{H}), 1.66-1.59(\mathrm{~m}$, $2 \mathrm{H}), 0.93(\mathrm{t}, 3 \mathrm{H}, J=7.4 \mathrm{~Hz}) ;{ }^{13} \mathrm{CNMR}\left(\mathrm{CDCl}_{3}, 101 \mathrm{MHz}\right) \delta 151.4,146.0,134.6,129.9$ (2C), 128.3 (2C), 78.8, 59.7, 52.7, 27.8, 21.7, 8.2; IR v max 2985, 2115, 1787, 1371, $1174 \mathrm{~cm}^{-1}$. Anal. Calcd. For $\mathrm{C}_{13} \mathrm{H}_{16} \mathrm{~N}_{4} \mathrm{O}_{4} \mathrm{~S}: \mathrm{C}, 48.14 ; \mathrm{H}, 4.97$; N, 17.27. Found: C, 48.39; H, 5.21; N, 17.08.

$\left({ }_{4} S^{*}, S^{*}\right)$-4-(Azidomethyl)-5-propyl-3-tosyloxazolidin-2-one (2h)

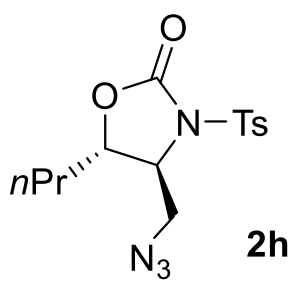

1h (297 mg); $\mathrm{PdCl}_{2}(\mathrm{MeCN})_{2}$ (13 mg); FCC-AcOEt/hexane (2:8). $2 \mathbf{h}$ (186 mg, 55\%, 24 h); white solid, m.p.: $74-75{ }^{\circ} \mathrm{C} .{ }^{1} \mathrm{H}$ NMR $\left(\mathrm{CDCl}_{3}, 400 \mathrm{MHz}\right) \delta 7.92$ $(\mathrm{d}, 2 \mathrm{H}, J=8.3 \mathrm{~Hz}), 7.36(\mathrm{~d}, 2 \mathrm{H}, J=8.2 \mathrm{~Hz}), 4.33-4.29(\mathrm{~m}, 1 \mathrm{H})$, 4.09-4.06

2h $(\mathrm{m}, 1 \mathrm{H}), 3.83(\mathrm{dd}, \mathrm{iH}, J=12.8,5.8), 3.68(\mathrm{dd}, 1 \mathrm{H}, J=12.8,2.8 \mathrm{~Hz}), 2.43(\mathrm{~s}$, $3 \mathrm{H}), 1.56-1.49(\mathrm{~m}, 2 \mathrm{H}), 1.37-1.27(\mathrm{~m}, 2 \mathrm{H}), 0.88(\mathrm{t}, 3 \mathrm{H}, J=7.2 \mathrm{~Hz}) ;{ }^{13} \mathrm{C} \mathrm{NMR}\left(\mathrm{CDCl}_{3}, 101 \mathrm{MHz}\right) \delta$ 
151.5, 146.0, 134.6, $129.9(2 \mathrm{C}), 128.3(2 \mathrm{C}), 77.6,60.2,52.6,32.6,21.7,17.3,13.5$; IR $v \max 2956$, 2114, 1772, 1244, $1139 \mathrm{~cm}^{-1}$. Anal. Calcd. For $\mathrm{C}_{14} \mathrm{H}_{18} \mathrm{~N}_{4} \mathrm{O}_{4} \mathrm{~S}: \mathrm{C}, 49.69 ; \mathrm{H}, 5.36 ; \mathrm{N}, 16.56$. Found: $\mathrm{C}$, $49 \cdot 58 ; \mathrm{H}, 5.27 ; \mathrm{N}, 16.68$.

4-(Hydroxymethyl)-5-propyl-3-tosyloxazolidin-2-one (3h)

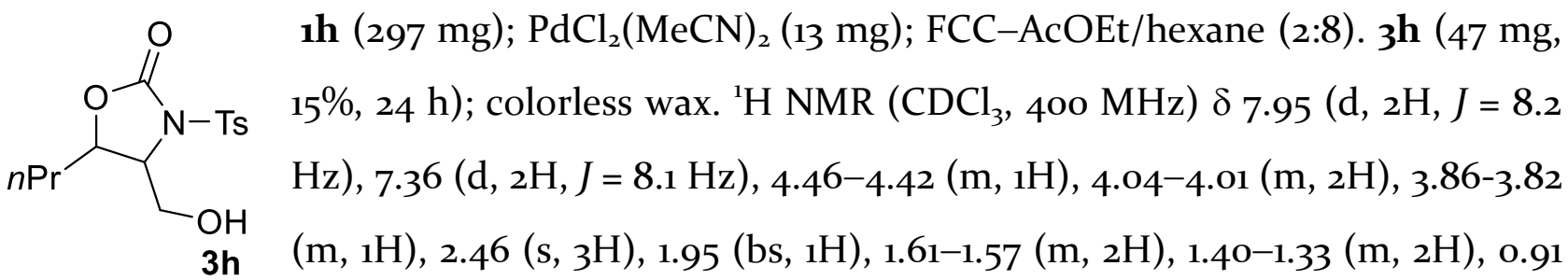
$(\mathrm{t}, 3 \mathrm{H}, J=7.3 \mathrm{~Hz}) ;{ }^{13} \mathrm{C} \mathrm{NMR}\left(\mathrm{CDCl}_{3}, 101 \mathrm{MHz}\right) \delta 152.1,141.1,131.8,129.9(2 \mathrm{C}), 128.4(2 \mathrm{C}), 77.3$, 63.1, 62.9, 36.7, 21.7, 17.5, 13.6; IR $v \max 3476,2961,1741,1354,1187 \mathrm{~cm}^{-1}$. Anal. Calcd. For: $\mathrm{C}_{14} \mathrm{H}_{19} \mathrm{NO}_{5} \mathrm{~S}: \mathrm{C}, 53.66 ; \mathrm{H}, 6.11 ; \mathrm{N}, 4.47$. Found: C, 53.97; H, 6.45; N, 4.18.

\section{4-(Azidomethyl)-5,5-dimethyl-3-tosyloxazolidin-2-one (2i)}

$\mathrm{O} \quad \mathbf{1 i}(283 \mathrm{mg}) ; \mathrm{PdCl}_{2}(\mathrm{MeCN})_{2}$ (13 mg); FCC-AcOEt/hexane (3:7). 2i (169 mg,

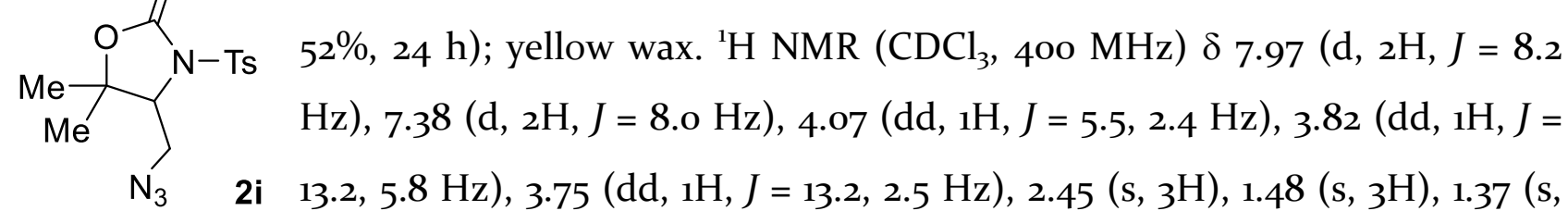
$3 \mathrm{H}) ;{ }^{13} \mathrm{C} \mathrm{NMR}\left(\mathrm{CDCl}_{3}, 101 \mathrm{MHz}\right) \delta 150.8,145.9,134.7,129.9(2 \mathrm{C}), 128.4(2 \mathrm{C}), 81.7,63.7,50.2$, 28.5, 21.7, 21.2; IR v max 3054, 2117, 1778, 1265, $1174 \mathrm{~cm}^{-1}$. Anal. Calcd. For $\mathrm{C}_{13} \mathrm{H}_{16} \mathrm{~N}_{4} \mathrm{O}_{4} \mathrm{~S}: \mathrm{C}, 48.14$; H, 4.97; N, 17.27. Found: C, 48.00; H, 4.86; N, 17.40.

\section{4-(Azidomethyl)-1-methyl-3-tosylimidazolidin-2-one (5a)}

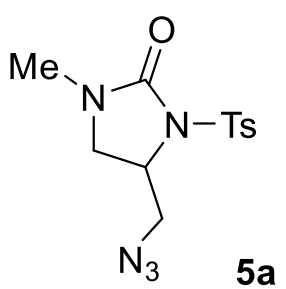

4a (268 mg); $\mathrm{PdCl}_{2}(\mathrm{MeCN})_{2}$ (13 mg); FCC-AcOEt/hexane (1:1). 5a (244 mg, $79 \%, 24 \mathrm{~h})$; colorless wax. ${ }^{1} \mathrm{H}$ NMR $\left(\mathrm{CDCl}_{3}, 400 \mathrm{MHz}\right) \delta 7.93(\mathrm{~d}, 2 \mathrm{H}, J=11.2$ $\mathrm{Hz}), 7.33(\mathrm{~d}, 2 \mathrm{H}, J=10.8 \mathrm{~Hz}), 4.41-4.33(\mathrm{~m}, 1 \mathrm{H}), 3.75-3.74(\mathrm{~m}, 2 \mathrm{H}), 3.51(\mathrm{t}$,

$\mathrm{NMR}\left(\mathrm{CDCl}_{3}, 101 \mathrm{MHz}\right) \delta$ 153.6, 144.9, 135.8, 129.6 (2C), 128.2 (2C), 53.6, 52.3, 47.6, 30.4, 21.6; IR $v_{\max } 2109,1769,1348,1169,1145,1122 \mathrm{~cm}^{-1}$. Anal. Calcd. For $\mathrm{C}_{12} \mathrm{H}_{15} \mathrm{~N}_{5} \mathrm{O}_{3} \mathrm{~S}: \mathrm{C}, 46.59 ; \mathrm{H}, 4.89$; N, 22.64. Found: 46.80; H, 5.11; N, 22.47. 
4-(Azidomethyl)-1-cyclohexyl-3-tosylimidazolidin-2-one (5b)

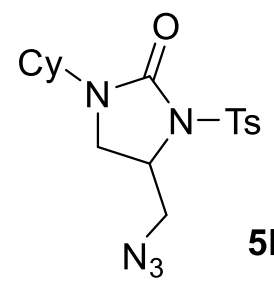

4b (336 mg); $\mathrm{Pd}(\mathrm{OAc})_{2}(16 \mathrm{mg}) ; \mathrm{FCC}-\mathrm{AcOEt} /$ hexane (3:7). 5b (294 mg, $78 \%, 24 \mathrm{~h})$; brown wax. ${ }^{1} \mathrm{H}$ NMR $\left(\mathrm{CDCl}_{3}, 400 \mathrm{MHz}\right) \delta 7.94(\mathrm{~d}, 2 \mathrm{H}, J=8.3$ $\mathrm{Hz}), 7.33(\mathrm{~d}, 2 \mathrm{H}, J=7.8 \mathrm{~Hz}), 4.41-4.36(\mathrm{~m}, 1 \mathrm{H}), 3.74-3.65(\mathrm{~m}, 3 \mathrm{H}), 3.48(\mathrm{t}$,

5b $\left.{ }_{1} \mathrm{H}, J=9.3 \mathrm{~Hz}\right), 3.21(\mathrm{dd}, 1 \mathrm{H}, J=9.3,3.9 \mathrm{~Hz}), 2.43(\mathrm{~s}, 3 \mathrm{H}), 1.78-1.62(\mathrm{~m}, 4 \mathrm{H})$, 1.34-1.27 (m, 6H); ${ }^{13} \mathrm{C} \mathrm{NMR}\left(\mathrm{CDCl}_{3}, 101 \mathrm{MHz}\right) \delta 152.7,144.8,136.0,129.6$ (2C), 128.2 (2C), 53.6, 52.5, 51.8, 41.7, 30.1 $\left(2 \mathrm{CH}_{2}\right), 29.8\left({ }_{2} \mathrm{CH}_{2}\right), 25.3,21.7$; IR $v \max 3053,2933,2110,1726,1265,1165$, $738 \mathrm{~cm}^{-1}$. Anal. Calcd. For $\mathrm{C}_{17} \mathrm{H}_{23} \mathrm{~N}_{5} \mathrm{O}_{3} \mathrm{~S}: \mathrm{C}, 54.09 ; \mathrm{H}, 6.14 ; \mathrm{N}, 18.55$. Found: $\mathrm{C}, 54.31 ; \mathrm{H}, 6.35 ; \mathrm{N}$, 18.30.

\section{4-(Azidomethyl)-1-phenyl-3-tosylimidazolidin-2-one (5c)}

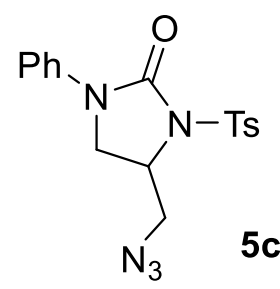

4c (330 mg); $\mathrm{Pd}(\mathrm{OAc})_{2}(16 \mathrm{mg}) ; \mathrm{FCC}-\mathrm{AcOEt} / \mathrm{hexane}(4: 6)$. 5c (171 mg, 46\% yield, 24 h); white solid, m.p.: $103-104{ }^{\circ} \mathrm{C} .{ }^{1} \mathrm{H} \mathrm{NMR}\left(\mathrm{CDCl}_{3}, 400 \mathrm{MHz}\right) \delta 7.99$ $(\mathrm{d}, 2 \mathrm{H}, J=8.2 \mathrm{~Hz}), 7.45(\mathrm{~d}, 2 \mathrm{H}, J=8.0 \mathrm{~Hz}), 7.36-7.31(\mathrm{~m}, 4 \mathrm{H}), 7.12(\mathrm{t}, 1 \mathrm{H}, J=$

$(\mathrm{dd}, 1 \mathrm{H}, J=9.5,3.5 \mathrm{~Hz}), 2.43(\mathrm{~s}, 3 \mathrm{H}) ;{ }^{13} \mathrm{C} \mathrm{NMR}\left(\mathrm{CDCl}_{3}, 101 \mathrm{MHz}\right) \delta 151.2,145.3,138.0,135.6$, 129.8 (2C), 129.1 (2C), 128.4 (2C), 124.6, 118.8 (2C), 53.6, 51.8, 46.3, 21.7; IR v max 2971, 2930, 2115, $1717,1409,1364,1349,1187 \mathrm{~cm}^{-1}$. Anal. Calcd. For $\mathrm{C}_{17} \mathrm{H}_{17} \mathrm{~N}_{5} \mathrm{O}_{3} \mathrm{~S}: \mathrm{C}, 54.98 ; \mathrm{H}, 4.61 ; \mathrm{N}, 18.86$. Found: C, 55.08; H, 4.69; N, 18.75 .

\section{4-(Hydroxymethyl)-1-phenyl-3-tosylimidazolidin-2-one (6c)}

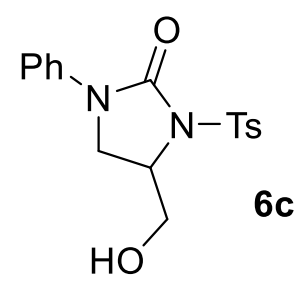

4c (330 mg); $\mathrm{Pd}(\mathrm{OAc})_{2}$ (16 mg); FCC-AcOEt/hexane (4:6). 6c(48 mg, 14\%, $24 \mathrm{~h})$; yellow wax. ${ }^{1} \mathrm{H}$ NMR $\left(\mathrm{CDCl}_{3}, 400 \mathrm{MHz}\right) \delta 7.98(\mathrm{~d}, 2 \mathrm{H}, J=8.2 \mathrm{~Hz})$,

6c $7.46(\mathrm{~d}, 2 \mathrm{H}, J=8.1 \mathrm{~Hz}), 7 \cdot 35-7.30(\mathrm{~m}, 4 \mathrm{H}), 7.10(\mathrm{t}, 1 \mathrm{H}, J=7.3 \mathrm{~Hz}), 4.47-4.44$ $(\mathrm{m}, 1 \mathrm{H}), 4.07(\mathrm{dd}, 1 \mathrm{H}, J=11.4,4.3 \mathrm{~Hz}), 4.01-3.93(\mathrm{~m}, 2 \mathrm{H}), 3.83(\mathrm{dd}, 1 \mathrm{H}, J=$ 9.2, 4.2 Hz), 2.43 (s, 3H), 1.59 (bs, $1 \mathrm{H}) .{ }^{13} \mathrm{C} \mathrm{NMR}\left(\mathrm{CDCl}_{3}, 101 \mathrm{MHz}\right) \delta$ 151.7, 145.1, 138.2, 135.6, 129.8 (2C), $129.0(2 \mathrm{C}), 128.3(2 \mathrm{C}), 124.4,118.7$ (2C), 64.0, 54.5, 45.7, 21.7; IR v max 3502, 2919, $1717,1597,1409,1346,1163,1087 \mathrm{~cm}^{-1}$. Anal. Calcd. For $\mathrm{C}_{17} \mathrm{H}_{18} \mathrm{~N}_{2} \mathrm{O}_{4} \mathrm{~S}: \mathrm{C}, 58.95 ; \mathrm{H}, 5.24 ; \mathrm{N}, 8.09$. Found: C, 59.17; H, 5.49; N, 7.91. 


\section{4-(Azidomethyl)-1-benzyl-3-tosylimidazolidin-2-one (5d)}

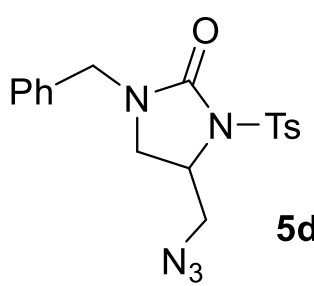

4d (344 mg); $\mathrm{Pd}(\mathrm{OAc})_{2}$ (16 mg); FCC-AcOEt/hexane (4:6). 5d (312 mg, 81\%, 24h); yellow wax. ${ }^{1} \mathrm{H}$ NMR $\left(\mathrm{CDCl}_{3}, 400 \mathrm{MHz}\right) \delta 7.85(\mathrm{~d}, 2 \mathrm{H}, J=7.9$ $\mathrm{Hz})$, 7.23-7.15 (m, 5H), 7.03-7.02 (m, 2H), 4.25-4.20 (m, $3 \mathrm{H}), 3.62$ (dd, $2.97(\mathrm{dd}, 1 \mathrm{H}, J=9.4,3.8 \mathrm{~Hz}), 2.34(\mathrm{~s}, 3 \mathrm{H}) ;{ }^{13} \mathrm{C} \mathrm{NMR}\left(\mathrm{CDCl}_{3}, 101 \mathrm{MHz}\right) \delta$ 153.6, 145.0, 135.8, 135.1, $129.7(2 \mathrm{C}), 128.8,128.2(2 \mathrm{C}), 128.1(2 \mathrm{C})$. $128.0(2 \mathrm{C})$, 53.7, 52.4, 47.6, 44.9, 21.7. Anal. Calcd. For $\mathrm{C}_{18} \mathrm{H}_{19} \mathrm{~N}_{5} \mathrm{O}_{3} \mathrm{~S}: \mathrm{C}, 56.09 ; \mathrm{H}, 4.97$; N, 18.17. Found: C, 56.38; H, 5.32; N, 17.90.

\section{(R)-4-(Azidomethyl)-1-[(R)-1-phenylethyl]-3-tosylimidazolidin-2-one (5e)}<smiles>C[C@H](c1ccccc1)N1C[C@H](CN)N([13CH3])C1=O</smiles>

4e (358 mg); $\mathrm{Pd}(\mathrm{OAc})_{2}(16 \mathrm{mg}) ; \mathrm{FCC}-\mathrm{AcOEt} /$ hexane (4:6). 5e (208 mg, $52 \% 24 \mathrm{~h})$; yellow wax, $[\alpha]_{\mathrm{D}}{ }^{2 \mathrm{O}}=+8.43\left(\mathrm{co} 0.7, \mathrm{CH}_{2} \mathrm{Cl}_{2}\right) .{ }^{1} \mathrm{H} \mathrm{NMR}\left(\mathrm{CDCl}_{3}\right.$, $400 \mathrm{MHz}) \delta 7.94(\mathrm{~d}, 2 \mathrm{H}, J=8.2 \mathrm{~Hz}), 7.36-7.28(\mathrm{~m}, 5 \mathrm{H}), 7.13(\mathrm{~d}, 2 \mathrm{H}, J=$ $7.9 \mathrm{~Hz}), 5.22(\mathrm{q}, 1 \mathrm{H}, J=7.0 \mathrm{~Hz}), 4.31-4.25(\mathrm{~m}, 1 \mathrm{H}), 3.75(\mathrm{dd}, 1 \mathrm{H}, J=12.5$, 6.0 Hz), 3.64 (dd, $1 \mathrm{H}, J=12.5,2.9 \mathrm{~Hz}), 3.14(\mathrm{dd}, 1 \mathrm{H}, J=9.4,3.9 \mathrm{~Hz}), 3.05(\mathrm{t}, 1 \mathrm{H}, J=9.4 \mathrm{~Hz})$, $2.46(\mathrm{~s}, 3 \mathrm{H}), 1.5 \mathrm{O}(\mathrm{d}, 3 \mathrm{H}, J=7.1 \mathrm{~Hz}) ;{ }^{13} \mathrm{C} \mathrm{NMR}\left(\mathrm{CDCl}_{3}, 101 \mathrm{MHz}\right) \delta 153.1,144.9,138.7,135.8,129.7$ (2C), $128.7(2 \mathrm{C}), 128.2(2 \mathrm{C})$, 127.9, $127.0(2 \mathrm{C})$, 53.8, 52.5, 50.4, 41.0, 21.7, 16.0; IR v max 3097, 2954, 2107, 1730, 1411, 1178, $809 \mathrm{~cm}^{-1}$. Anal. Calcd. For $\mathrm{C}_{19} \mathrm{H}_{21} \mathrm{~N}_{5} \mathrm{O}_{3} \mathrm{~S}: \mathrm{C}, 57.13 ; \mathrm{H}, 5.30 ; \mathrm{N}, 17.53$. Found: C, 57.44; H, 5.58; N, $17.33 .{ }^{[15]}$

\section{2-Azidomethyl-1-tosylindoline (8a)}

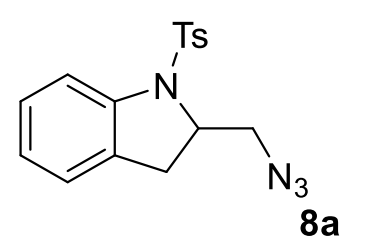

7a (287 mg); $\mathrm{Pd}(\mathrm{OAc})_{2}$ (16 mg); FCC-AcOEt/hexane (3:7). 8a (154 mg, $47 \%, 48 \mathrm{~h})$; colorless wax. ${ }^{1} \mathrm{H}$ NMR $\left(\mathrm{CDCl}_{3}, 400 \mathrm{MHz}\right) \delta 7.70\left(\mathrm{~d},{ }_{1} \mathrm{H}, J=\right.$ $7.9 \mathrm{~Hz}), 7.56(\mathrm{~d}, 2 \mathrm{H}, J=8.0 \mathrm{~Hz}), 7.61-7.07(\mathrm{~m}, 3 \mathrm{H}), 7.09(\mathrm{~d}, 2 \mathrm{H}, J=2.3$ $\mathrm{Hz}), 4.38-4.35(\mathrm{~m}, 1 \mathrm{H}), 3.64(\mathrm{dd}, 1 \mathrm{H}, J=4.6,3.8 \mathrm{~Hz}), 3.56(\mathrm{dd}, 1 \mathrm{H}, J=$ 12.2, $7.2 \mathrm{~Hz}), 2.88(\mathrm{dd}, 1 \mathrm{H}, J=16.5,9.1 \mathrm{~Hz}) 2.75(\mathrm{~d}, 1 \mathrm{H}, J=12.8 \mathrm{~Hz}), 2.38(\mathrm{~s}, 3 \mathrm{H})$. The characterization of product $\mathbf{8 a}$ is consistent with that reported in the literature. ${ }^{[16]}$

[15]Major diasteroisomer (isolated yield); minor diasteroisomer not isolated; diastereomeric ratio 78:22 determined by $1 \mathrm{H}-\mathrm{NMR}$ analysis of the crude reaction mixture.

[16] Sequeira, F. C.; Turpenny, B. W.; Chemler, S. M. Angew. Chem. Int. Ed., 2010, 49, 6353 


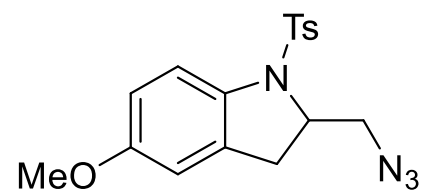

7b (317 mg); Pd(OAc) (16 mg); FCC-AcOEt/hexane (4:6). 8b (183 mg, $51 \%, 48 \mathrm{~h})$; colorless wax. ${ }^{1} \mathrm{H} \mathrm{NMR}\left(\mathrm{CDCl}_{3}, 400 \mathrm{MHz}\right) \delta 7.60$ $(\mathrm{d}, 1 \mathrm{H}, J=8.8 \mathrm{~Hz}), 7.49(\mathrm{~d}, 2 \mathrm{H}, J=8.0 \mathrm{~Hz}), 7.17(\mathrm{~d}, 2 \mathrm{H}, J=8.0 \mathrm{~Hz})$,

8b $6.77(\mathrm{~d}, 1 \mathrm{H}, J=8.7 \mathrm{~Hz}), 6.59(\mathrm{~s}, 1 \mathrm{H}), 4.27-4.26(\mathrm{~m}, 1 \mathrm{H}), 3.76(\mathrm{~s}, 3 \mathrm{H})$, $3.67(\mathrm{~d}, 2 \mathrm{H}, J=5.8 \mathrm{~Hz}), 2.69(\mathrm{dd}, 1 \mathrm{H}, J=16.3,9.5 \mathrm{~Hz}), 2.51(\mathrm{~d}, 1 \mathrm{H}, J=16.3 \mathrm{~Hz}), 2.36(\mathrm{~s}, 3 \mathrm{H}) ;{ }^{13} \mathrm{C}$ NMR $\left(\mathrm{CDCl}_{3}, 101 \mathrm{MHz}\right) \delta$ 157.7, 144.1, 134.5, 133.9, 130.2, 129.6 (2C), 127.3 (2C), 119.0, 113.0, 110.1, 65.4, 63.9, 55.6, 31.4, 21.6; IR $v \max 3258,2104,1452,1259,1174,806 \mathrm{~cm}^{-1}$. Anal. Calcd. For $\mathrm{C}_{17} \mathrm{H}_{18} \mathrm{~N}_{4} \mathrm{O}_{3} \mathrm{~S}: \mathrm{C}, 56.97 ; \mathrm{H}, 5.06 ; \mathrm{N}, 15.63$. Found: $\mathrm{C}, 57.33 ; \mathrm{H}, 5.42 ; \mathrm{N}, 15.31$.

\section{5-(Azidomethyl)-1-cyclohexyl-4-tosylpiperazin-2-one (10a)}<smiles>[13CH3]N1CC(=O)N([Al])CC1CN</smiles>

9a (344 mg); Pd(OAc) 2 (16mg); FCC-AcOEt/hexane (1:1). 10a (203 mg, 52\%, $48 \mathrm{~h})$; yellow wax. ${ }^{1} \mathrm{H} \mathrm{NMR}\left(\mathrm{CDCl}_{3}, 400 \mathrm{MHz}\right) \delta 7.71(\mathrm{~d}, 2 \mathrm{H}, J=7.8 \mathrm{~Hz})$, $7.33(\mathrm{~d}, 2 \mathrm{H}, J=7.8 \mathrm{~Hz}), 4.23-4.15(\mathrm{~m}, 1 \mathrm{H}), 4.08(\mathrm{~d}, 1 \mathrm{H}, J=16.6 \mathrm{~Hz}), 3.93-3.87$ $(\mathrm{m}, 1 \mathrm{H}), 3.78(\mathrm{~d}, 1 \mathrm{H}, J=16.6 \mathrm{~Hz}), 3.56(\mathrm{dd}, 1 \mathrm{H}, J=12.3,4.6 \mathrm{~Hz}), 3.45(\mathrm{dd}, 1 \mathrm{H}$, $J=12.3,8.8 \mathrm{~Hz}), 3.26(\mathrm{dd}, 1 \mathrm{H}, J=13.6,4.6 \mathrm{~Hz}), 3.16(\mathrm{dd}, 1 \mathrm{H}, J=13.6,4.9 \mathrm{~Hz})$, $2.42(\mathrm{~s}, 3 \mathrm{H}), 1.78-1.61(\mathrm{~m}, 4 \mathrm{H}), 1.35-1.26(\mathrm{~m}, 3 \mathrm{H}), 1.22-0.3(\mathrm{~m}, 3 \mathrm{H}) .{ }^{13} \mathrm{C} \mathrm{NMR}\left(\mathrm{CDCl}_{3}, 101 \mathrm{MHz}\right) \delta$ 164.6, 144.5, 134.6, 130.0 (2C), 127.6 (2C), 52.0, 51.8, 51.2, 46.8, 40.8, 29.6, 29.5, 25.4, 25.3, 25.2, 21.5; IR $v \max 2933,2858,2108.1668,1451,1353,1265,1165,737 \mathrm{~cm}^{-1}$. Anal. Calcd. For $\mathrm{C}_{18} \mathrm{H}_{25} \mathrm{~N}_{5} \mathrm{O}_{3} \mathrm{~S}: \mathrm{C}, 55.22 ; \mathrm{H}, 6.44 ; \mathrm{N}, 17.89$. Found: C, 55.51; H, 6.77; N, 17.61.

\section{5-(Azidomethyl)-1-phenyl-4-tosylpiperazin-2-one (1ob)}

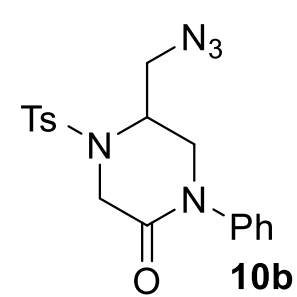

9b (344 mg); Pd(OAc) 2 (16 mg,); FCC-AcOEt/hexane (6:4). 1ob (285 mg, $74 \%, 48 \mathrm{~h}$ ); colorless wax. ${ }^{1} \mathrm{H}$ NMR $\left(\mathrm{CDCl}_{3}, 400 \mathrm{MHz}\right) \delta 7.78(\mathrm{~d}, 2 \mathrm{H}, J=8.3$ $\mathrm{Hz}), 7.38(\mathrm{~d}, 2 \mathrm{H}, J=8.3 \mathrm{~Hz}), 7.33-7.22(\mathrm{~m}, 3 \mathrm{H}), 6.86(\mathrm{~d}, 2 \mathrm{H}, J=7.8 \mathrm{~Hz}), 4.31$

$(\mathrm{d}, 1 \mathrm{H}, J=17.1 \mathrm{~Hz}), 4.11-4.06(\mathrm{~m}, 1 \mathrm{H}), 3.98(\mathrm{~d}, 1 \mathrm{H}, J=17.1 \mathrm{~Hz}), 3.73-3.67(\mathrm{~m}$, $4 \mathrm{H}), 2.47(\mathrm{~s}, 3 \mathrm{H}) .{ }^{13} \mathrm{C} \mathrm{NMR}\left(\mathrm{CDCl}_{3}, 101 \mathrm{MHz}\right) \delta 165.3,144.7,140.6,134.5,130.2(2 \mathrm{C}), 129.2(2 \mathrm{C})$, $127.6(2 \mathrm{C}), 127.2,124.9(2 \mathrm{C}), 52.4,52.0,49.5,47.4,21.6$; IR $v \max 3055,2109,1691,1596,1265$, 1164, $737 \mathrm{~cm}^{-1}$. Anal. Calcd. For $\mathrm{C}_{18} \mathrm{H}_{19} \mathrm{~N}_{5} \mathrm{O}_{3} \mathrm{~S}$ : C, 56.09; H, 4.97; N, 18.17. Found: $\mathrm{C}, 56.24 ; \mathrm{H}$, $5.13 ; \mathrm{N}, 18.04$. 
3-(Azidomethyl)-4-tosyl-3,4-dihydro-2H-benzo[b][1,4]oxazine (12)

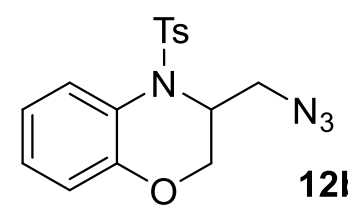

11 (303 mg); $\mathrm{Pd}(\mathrm{OAc})_{2}$ (16 mg); FCC-AcOEt/hexane (1:9). 11 (269 mg, $78 \%, 48 \mathrm{~h})$; colorless wax. ${ }^{1} \mathrm{H}$ NMR $\left(\mathrm{CDCl}_{3}, 300 \mathrm{MHz}\right) \delta 7.92(\mathrm{dd}, 1 \mathrm{H}, J=$ 12b $8.2,1.1 \mathrm{~Hz}), 7.48(\mathrm{~d}, 2 \mathrm{H}, J=8.2 \mathrm{~Hz}), 7.23(\mathrm{~d}, 2 \mathrm{H}, J=8.1 \mathrm{~Hz}), 7.11-7.05(\mathrm{~m}$, $\left.{ }_{1 \mathrm{H}}\right)$, 6.99-6.94 (m, $\left.1 \mathrm{H}\right), 6.78\left(\mathrm{dd},{ }_{1} \mathrm{H}, J=8.1,1.1 \mathrm{~Hz}\right), 4.41-4.34(\mathrm{~m}, 1 \mathrm{H}), 4.10(\mathrm{dd}, 1 \mathrm{H}, J=11.5$, $0.9 \mathrm{~Hz}), 3.67(\mathrm{dd}, 1 \mathrm{H}, J=11.2,7.7 \mathrm{~Hz}), 3.54(\mathrm{dd}, 1 \mathrm{H}, J=11.2,7.1 \mathrm{~Hz}), 3.17(\mathrm{dd}, 1 \mathrm{H}, J=11.5,2.9$ $\mathrm{Hz}), 2.39$ (s, $3 \mathrm{H}) ;{ }^{13} \mathrm{C} \mathrm{NMR}\left(\mathrm{CDCl}_{3}, 75 \mathrm{MHz}\right) \delta 146.1,144.4,135.0,129.9$ (2C), $127.3(2 \mathrm{C}), 126.4$, 125.6, 121.9, 121.4, 117.3, 61.8, 60.4, 54.3, 21.5; IR $v \max 3231,2112,1438,1271,1166,837 \mathrm{~cm}^{-1}$. Anal. Calcd. For $\mathrm{C}_{16} \mathrm{H}_{16} \mathrm{~N}_{4} \mathrm{O}_{3} \mathrm{~S}$ : C, 55.80; H, 4.68; N, 16.27. Found: C, 55.68; H, 4.57; N, 16.36.

2-(Azidomethyl)-4-methyl-1-tosyl-1,2,3,4-tetrahydro-5H-benzo[e] $[1,4]$ diazepin-5-one (14a)

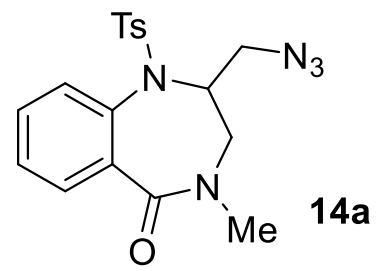

13a (344 mg, $0.357 \mathrm{mmol}) ; \mathrm{Pd}(\mathrm{OAc})_{2}$ (16 mg); FCC-AcOEt/hexane (4:6). 14a (193 mg, 50\%, $48 \mathrm{~h}$ ); white solid, m.p.: $156-159{ }^{\circ} \mathrm{C} .{ }^{1} \mathrm{H}$ NMR $\left(\mathrm{CDCl}_{3}, 400 \mathrm{MHz}\right) \delta 7.56(\mathrm{dd}, 1 \mathrm{H}, J=7.3,1.6 \mathrm{~Hz}), 7.50-7.47(\mathrm{~m}, 2 \mathrm{H})$, 7.45-7.37 (m, $1 \mathrm{H}), 7.34(\mathrm{~d}, 2 \mathrm{H}, J=8.3 \mathrm{~Hz}), 7.18(\mathrm{~d}, 2 \mathrm{H}, J=8.8 \mathrm{~Hz})$, 4.47-4.40 (m, $1 \mathrm{H}), 3.62(\mathrm{dd}, 1 \mathrm{H}, J=12.3,4.2 \mathrm{~Hz}), 3.41(\mathrm{dd}, 1 \mathrm{H}, J=12.3,7.3 \mathrm{~Hz}), 3.20(\mathrm{dd}, 1 \mathrm{H}, J=$ 15.2, $12.2 \mathrm{~Hz}), 3.07(\mathrm{dd}, \mathrm{lH}, J=15.2,4.9 \mathrm{~Hz}), 2.53(\mathrm{~s}, 3 \mathrm{H}), 2.35(\mathrm{~s}, 3 \mathrm{H}) ;{ }^{13} \mathrm{C} \mathrm{NMR}\left(\mathrm{CDCl}_{3}, 101\right.$ MHz) $\delta 167.5,144.1,135.3,134.4$, 133.1, 132.8, 132.0, 130.2, 129.9 (2C), 129.4, 127.2 (2C), 60.6, 53.5, 50.7, 34.2, 21.6; IR $v \max 2989,2110,1644,1463,1377,1352,1160 \mathrm{~cm}^{-1}$. Anal. Calcd. For $\mathrm{C}_{18} \mathrm{H}_{19} \mathrm{~N}_{5} \mathrm{O}_{3} \mathrm{~S}: \mathrm{C}, 56.09 ; \mathrm{H}, 4.97 ; \mathrm{N}, 18.17$. Found: C, 56.01; H, 4.92; N, 18.24 .

\section{2-(Azidomethyl)-4-benzyl-1-tosyl-1,2,3,4-tetrahydro-5H-benzo[e][1,4]diazepin-5-one} $(\mathbf{1 4 b})$

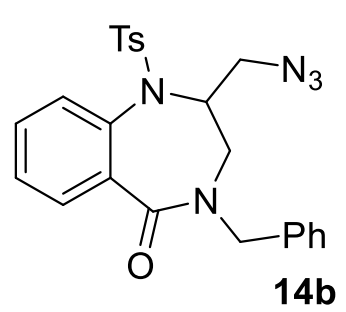

13b (421 mg); $\mathrm{Pd}(\mathrm{OAc})_{2}(16 \mathrm{mg}) ; \mathrm{FCC}-\mathrm{AcOEt} /$ hexane (4:6). 14b (323 mg, 70\%, 48 h); yellow solid, m.p.: $158-160{ }^{\circ} \mathrm{C} .{ }^{1} \mathrm{H}$ NMR $\left(\mathrm{CDCl}_{3}, 400 \mathrm{MHz}\right) \delta$ $7.72\left(\mathrm{~d},{ }_{1} \mathrm{H}, J=7.4 \mathrm{~Hz}\right), 7.59-7.44\left(\mathrm{~m},{ }_{5} \mathrm{H}\right), 7.32-7.28\left(\mathrm{~m},{ }_{5} \mathrm{H}\right), 7.12(\mathrm{~d}, 2 \mathrm{H}$, $J=6.5 \mathrm{~Hz}), 4.67(\mathrm{~d}, 1 \mathrm{H}, J=14.8 \mathrm{~Hz}), 4.50-4.43\left(\mathrm{~m},{ }_{1} \mathrm{H}\right), 3.62(\mathrm{dd}, 1 \mathrm{H}, J=$ $12.4,4.3 \mathrm{~Hz}), 3.51(\mathrm{~d}, 1 \mathrm{H}, J=15.0 \mathrm{~Hz}), 3.46(\mathrm{dd}, 1 \mathrm{H}, J=12.4,7.2 \mathrm{~Hz}), 3.16-$ $3.03(\mathrm{~m}, 2 \mathrm{H}), 2.43(\mathrm{~s}, 3 \mathrm{H}) ;{ }^{13} \mathrm{C}$ NMR $\left(\mathrm{CDCl}_{3}, 101 \mathrm{MHz}\right) \delta 167.6,144.2,136.3,135.4,134.4$, 133.0, 132.9, 132.1, 130.5, 130.0 (2C), 129.4, 128.8 (2C), 128.1 (2C), 127.9, 127.3 (2C), 6o.6, 53.5, 49.3, 47.4, 21.6; IR $v \max 2927,2088,1640,1598,1355,1263,1107,1101 \mathrm{~cm}^{-1}$. Anal. Calcd. For $\mathrm{C}_{24} \mathrm{H}_{23} \mathrm{~N}_{5} \mathrm{O}_{3} \mathrm{~S}: \mathrm{C}, 62.46 ; \mathrm{H}, 5.02 ; \mathrm{N}, 15.17$. Found: $\mathrm{C}, 62.62 ; \mathrm{H}, 5.20 ; \mathrm{N}, 15.05$. 
2-(Azidomethyl)-4-cyclohexyl-1-tosyl-1,2,3,4-tetrahydro-5 $H$-benzo[e][1,4]diazepin-5one $(14 \mathrm{c})$<smiles>CC1CN([AlH2])C(=O)c2ccccc2N1[AsH]</smiles>
13C (413 mg); Pd(OAc) 2 (16 mg); FCC-AcOEt/hexane (1:1). 14c (263 mg, $58 \%, 48 \mathrm{~h})$; white solid, m.p.: $156-157^{\circ} \mathrm{C} .{ }^{1} \mathrm{H}$ NMR $\left(\mathrm{CDCl}_{3}, 400 \mathrm{MHz}\right) \delta$ $7.68(\mathrm{~d}, 1 \mathrm{H}, J=7.4 \mathrm{~Hz}), 7.53-7.45\left(\mathrm{~m},{ }_{5} \mathrm{H}\right), 7.23(\mathrm{~d}, 2 \mathrm{H}, J=8.1 \mathrm{~Hz})$,

4.22-4.15 (m, $1 \mathrm{H}), 3.92-3.86(\mathrm{~m}, 1 \mathrm{H}), 3.72\left(\mathrm{dd},{ }_{1} \mathrm{H}, J=12.3,4.4 \mathrm{~Hz}\right), 3.43$ (dd, $1 \mathrm{H}, J=12.3,8.0 \mathrm{~Hz}$ ), $3.32(\mathrm{dd}, 1 \mathrm{H}, J=15.4,4.6 \mathrm{~Hz}), 2.93(\mathrm{dd}, 1 \mathrm{H}, J=15.4,12.0 \mathrm{~Hz}), 2.41$ (s, $3 \mathrm{H}), 1.74-1.71(\mathrm{~m}, 2 \mathrm{H}), 1.40^{-1.30}(\mathrm{~m} .2 \mathrm{H}), 1.18-1.03(\mathrm{~m}, 6 \mathrm{H}) ;{ }^{13} \mathrm{C} \mathrm{NMR}\left(\mathrm{CDCl}_{3}, 101 \mathrm{MHz}\right) \delta$ $167.6,144.1,135.8,135.2,132.8,132.5,131.7,130.6,129.7(2 \mathrm{C}), 129.2,127.7(2 \mathrm{C}), 62.9,53.3,52.8$, 43.5, 30.4, 30.2, 25.7, 25.4, 25.3, 21.6; IR v $\max 2936,2853,2093,1638,1460,1345,1168,997 \mathrm{~cm}^{-1}$. Anal. Calcd. For $\mathrm{C}_{23} \mathrm{H}_{27} \mathrm{~N}_{5} \mathrm{O}_{3} \mathrm{~S}$ : C, 6o.91; H, 6.00; N, 15.44. Found: C, 61.01; H, 6.05; N, 15.40 .

4-Cyclohexyl-2-(hydroxymethyl)-1-tosyl-1,2,3,4-tetrahydro-5H-benzo[e][1,4]diazepin5-one (15c)<smiles>O=C1c2ccccc2N([12F])C(CO)CN1C1CCCCC1</smiles>

13c (413 mg, $0.533 \mathrm{mmol}) ; \mathrm{Pd}(\mathrm{OAc})_{2}$ (16 mg); FCC-AcOEt/hexane (1:1). 15C (103 mg, 24\%, 48 h); white solid, m.p.: 164-165 ${ }^{\circ} \mathrm{C} .{ }^{1} \mathrm{H}-\mathrm{NMR}$ $\left(\mathrm{CDCl}_{3}, 400 \mathrm{MHz}\right): \delta 7.69(\mathrm{~d}, 1 \mathrm{H}, J=7.4 \mathrm{~Hz}), 7.55^{-7.44}\left(\mathrm{~m},{ }_{5} \mathrm{H}\right), 7.24$ $(\mathrm{d}, 2 \mathrm{H}, J=8.0 \mathrm{~Hz}), 4.19-4.12(\mathrm{~m}, 1 \mathrm{H}), 3.89-3.84(\mathrm{~m}, 1 \mathrm{H}), 3.76(\mathrm{dd}, 1 \mathrm{H}, J$ = 11.1, 6.2 Hz), 3.58-3.54 (m, $1 \mathrm{H}), 3.34(\mathrm{dd}, 1 \mathrm{H}, J=15.4,4.7 \mathrm{~Hz}), 2.88(\mathrm{dd}, 1 \mathrm{H}, J=15.4,12.1 \mathrm{~Hz})$, $2.41(\mathrm{~s}, 3 \mathrm{H}), 2.30\left(\mathrm{bs},{ }_{1} \mathrm{H}\right), 1.72-1.57(\mathrm{~m}, 6 \mathrm{H}), 1.14-1.00(\mathrm{~m}, 4 \mathrm{H}) ;{ }^{13} \mathrm{C} \mathrm{NMR}\left(\mathrm{CDCl}_{3}, 101 \mathrm{MHz}\right) \delta$ 167.8, 144.1, 136.0, 135.3, 132.8, 132.7, 131.6, 130.5, 129.7 (2C), 129.2, 127.9 (2C), 65.6, 63.1, 52.8, 43.0, 30.4, 30.1, 25.7, 25.4, 25.3, 21.6; IR $v \max 3355$, 2930, 2854, 1609, 1469, 1361, 1164, 1053, 989 $\mathrm{cm}^{-1}$. Anal. Calcd. For $\mathrm{C}_{23} \mathrm{H}_{28} \mathrm{~N}_{2} \mathrm{O}_{4} \mathrm{~S}: \mathrm{C}, 64.46 ; \mathrm{H}, 6.59 ; \mathrm{N}, 6.54$. Found: C, 64.55; H, [6.64; N, 6.41 .

\section{Preparation of 4-(1-azidoethyl)-3-tosyloxazolidin-2-one (16)}<smiles>[3H]N1C(=O)OCC1C(C)N</smiles>

To a solution of allyl derivative $\mathbf{1 j}(269 \mathrm{mg}, \mathbf{1 . 0} \mathrm{mmol})$ in THF (4 mL) were added in sequence: PIFA (473 mg, $1.1 \mathrm{mmol}$ ), KI (332 mg, 2.0 mmol.), $\mathrm{NaN}_{3}$ (195 mg, $3.0 \mathrm{mmol}$ ). The resulting solution was stirred at $25{ }^{\circ} \mathrm{C}$ until 16 completion (48 h; TLC control - AcOEt/hexane 1:1), then, the crude mixture 
was evaporated under reduced pressure and the residue was purified by FCC (AcOEt/hexane 4:6) to afford compound 16 (130 mg, 42\%); colorless wax. ${ }^{[17]}{ }^{1} \mathrm{H}$ NMR (40o $\left.\mathrm{MHz}, \mathrm{CDCl}_{3}\right) \delta$ $7.95(\mathrm{~d}, 2 \mathrm{H}, J=8.3 \mathrm{~Hz}), 7.36$ (d, $2 \mathrm{H}, J=8.1 \mathrm{~Hz}), 4.51^{-4} .49(\mathrm{~m}, 1 \mathrm{H}), 4.41^{-} 4.38(\mathrm{~m}, 2 \mathrm{H}), 4.30(\mathrm{t}$,

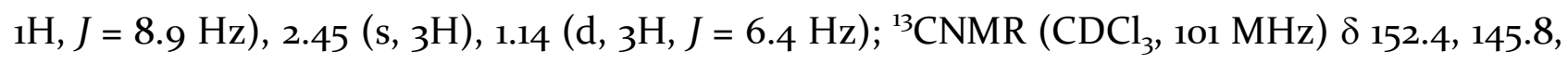
134.7, 129.8 (2C), 128.5 (2C), 66.9, 64.1, 60.0, 21.7, 16.3; IR $v_{\max } 2112,1768,1344,1190,1170,1131$ $\mathrm{cm}^{-1}$. Anal. Calcd. For $\mathrm{C}_{12} \mathrm{H}_{14} \mathrm{~N}_{4} \mathrm{O}_{4} \mathrm{~S}: \mathrm{C}, 46.45 ; \mathrm{H}, 4.55 ; \mathrm{N}, 18.05$. Found: $\mathrm{C}, 46.73 ; \mathrm{H}, 4.76 ; \mathrm{N}$, 17.86 .

[17] Although we cannot definitively rule out the formation of a mixture of two diastereoisomers, the ${ }^{1} \mathrm{H}$ - and ${ }^{13} \mathrm{C}-\mathrm{NMR}$ spectrum of the product, isolated as a single spot by chromatography, shows only one set of signals. 


\section{X-ray crystallography}

\section{Experimentals}

\section{Single-crystal X-ray diffraction analysis of $2 \mathrm{~h}$}

X-ray quality specimens of $\mathbf{2 h}$ were obtained by slow evaporation at $\mathrm{RT}$ from $\mathrm{CH}_{2} \mathrm{Cl}_{2}$. The crystalline precipitate was put into a few $\mathrm{mL}$ of $\mathrm{n}$-hexane to avoid contact with air. Crystals were stored a refrigerator at $\mathrm{T}=4{ }^{\circ} \mathrm{C}$ prior performing the analysis, but no specific precautions were adopted during the data collection. The specimen (Figure S1) was a colourless, transparent prism with dimensions $0.225 \times 0.225 \times 0.375 \mathrm{~mm}$. It was cut from a large aggregate with a stainless microblade and polished by mechanical ablation in a drop of perfluorinated oil. It showed pleochroism from colourless to dark grey under polarized light. A small epitaxial twinning was also detected and accounted for (see below).

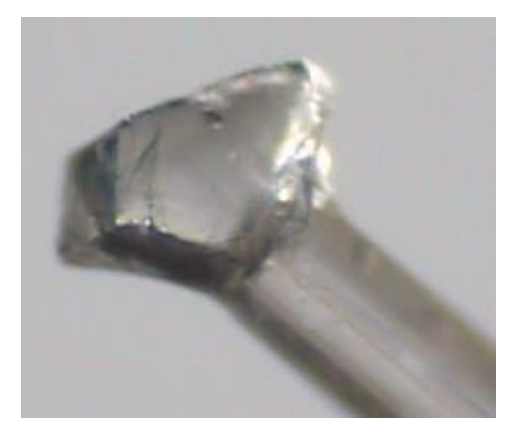

Figure S-1. Sample of $\mathbf{2 h}$ employed for the X-ray analysis, mounted on the top of a capillary fibre with a drop of perfluorinated oil.

The experiment explored a $97.5 \%$ complete sphere of data up to $61.3 \mathrm{deg}$ of $2 \theta$, with $R_{\text {int }}=0.0185$. 5019 symmetry-independent reflections were obtained, 4091 of which being highly significant (I > $2 \sigma(\mathrm{I}))$. Data reduction was carried with the SAINT+ ${ }^{[18]}$ suite of programs, while TWINABS ${ }^{[19]}$ dealt with absorption correction and scaling. The structure was solved in the shelxs environment by means of direct methods and refined within the Independent Atom Model (IAM) approximation with shelxl. ${ }^{[20]}$

[18] Bruker (2012). SAINT+. Bruker AXS Inc., Madison, Wisconsin, USA

[19] Bruker (2001). TWINABS. Bruker AXS Inc., Madison, Wisconsin, USA

[20] Sheldrick, G. M. Acta Cryst. 2015, $C_{71}, 3-8$ 
The compound $\mathbf{2 h}$ crystallizes as a 1:1 racemate in the centric $\mathrm{P} 2{ }_{1} / n\left(\mathrm{n}^{\mathrm{o}} 13\right)$ space group, with one molecule per asymmetric unit (Figure S2). The relative configuration of the two chiral centres was unequivocally determined as $\mathrm{R} / \mathrm{R}(\mathrm{C} 2 / \mathrm{C} 3) \mathrm{S} / \mathrm{S}(\mathrm{C} 2 / \mathrm{C} 3)$ for the two enantiomers.

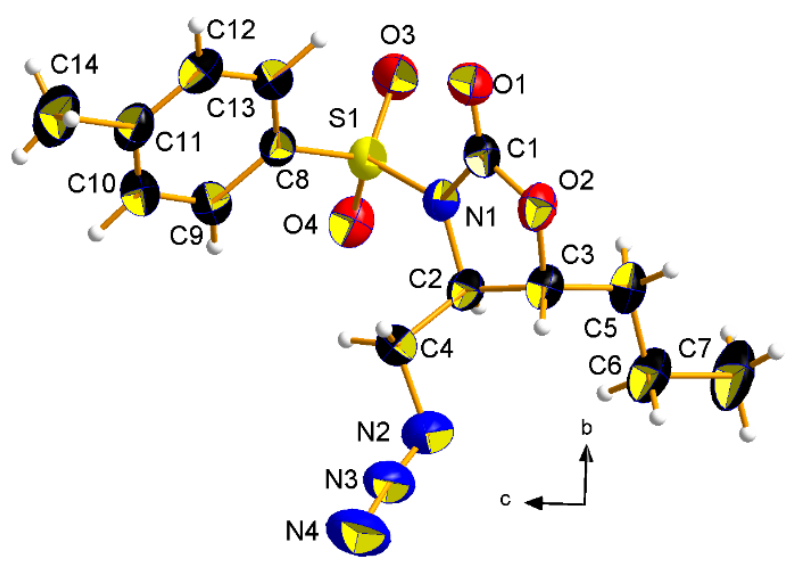

Figure S-2. Asymmetric unit of $\mathbf{2 h}$ at RT, with the atom-numbering scheme and the crystallographic reference system highlighted. Thermal ellipsoids of non- $\mathrm{H}$ atoms were drawn at the $50 \%$ probability level. The usual colour code was employed to draw atoms (black: C; white: H; red: O; blue: N; yellow: S).

The cell edges are $\mathrm{a}=10.2410(3) \AA, \mathrm{b}=10.0679(3) \AA, \mathrm{c}=16.3158(6) \AA, \beta=96.402(1) \operatorname{deg}, \mathrm{V}=$ $1636.4(2) \AA^{3}$, and density $=1.344 \mathrm{~g} / \mathrm{cm}^{3}$. The final least-squares model refines to an agreement factor $R_{1}(F)=0.0386$ for 4091 data with $F_{\mathrm{o}}>4 \sigma\left(F_{\mathrm{o}}\right)$, and $R_{1}(F)=0.0472$ for all the independent data, with goodness-of-fit as low as 1.049 and maximum and minimum Fourier difference peaks of $+0.24 /-0.24 \mathrm{e} / \AA^{3}$. CCDC 1970338 contains the supplementary crystallographic data for this paper. These data can be obtained free of charge from The Cambridge Crystallographic Data Centre via www.ccdc.cam.ac.uk/structures.

The diffraction pattern reveals the occurrence of a weak epitaxial twinning, whose lattice is rotated by $174.7^{\circ}$ around the [010] direction. The analysis of the diffraction intensities quantifies the relative weights of the two components as $99.4 \%$ and $0.6 \%$. Moreover, only a minority of the recorded intensities (22 reflections) are contaminated by twins overlap. We thus deemed not necessary to explicitly include the minority component into the least-squares procedure, and the structural model was retrieved by standard fitting against the structure factor squared amplitudes of the main lattice (HKLF 4). However, the quality of the final structural model is high, as testified by general fitting statistics and reasonable geometrical parameters. In particular, the covalent $\mathrm{N}-\mathrm{N}$ bonds in the azide group are asymmetric, as expected, with $\mathrm{d}_{\mathrm{N} 2-\mathrm{N} 3}=1.235(2) \AA$ and $\mathrm{d}_{\mathrm{N} 3-\mathrm{N} 4}=$ 1.132(2) ^, N4 being the terminal atom (see Figure S2). 
No strong hydrogen bond donors are present in $\mathbf{2 h}$, so only weak $\mathrm{CH} \cdots \mathrm{O}$ and $\mathrm{CH} \cdots \mathrm{N}$ contacts can be set up, which involve the $\mathrm{SO}_{2}$ moiety and the terminal $\mathrm{N} 4$ atom of the azide group. Inversionrelated molecules are arranged in a head-to-tail fashion (Figure S3a), with the aliphatic chain extending on the opposite direction of the tosyl protecting group along the $c$ cell axis. Azide chains occupy the free space along the orthogonal direction $b$ (Figure $\mathrm{S} 3 \mathrm{~b}$ ).

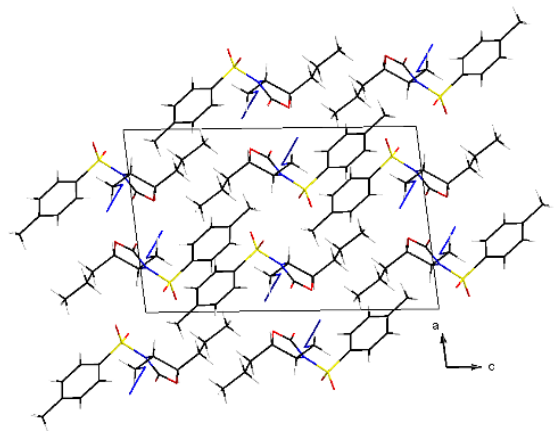

(a)

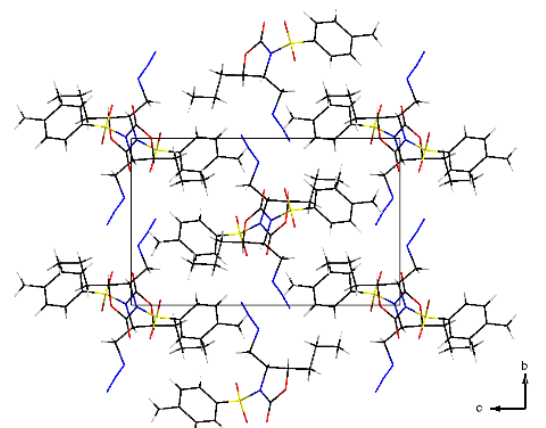

(b)

Figure S-3. Wires-stick representation of the crystal packing of $\mathbf{2 h}$, as seen along the monoclinic axis (a) and along the $a$ cell axis (b).

\section{Single-crystal X-ray diffraction analysis of $5 e$}

Crystals of 5e were obtained by slow evaporation at RT, with a procedure analogue to that employed for the $\mathbf{2 h}$ compound (see above). The habit is now acicular; after careful inspection at 40x magnification on a Stemi Zeiss stereomicroscope, a X-ray quality specimen was cut from a bundle of parallel needles with a stainless microblade. The selected crystal is colourless, transparent, with dimensions $0.600 \times 0.050 \times 0.025 \mathrm{~mm}$ (Figure S4) and shows pleochroism from colourless to dark grey under polarized light. However, it bore a neatly detectable but irreducible penetration twinning. Better quality specimens were not found.

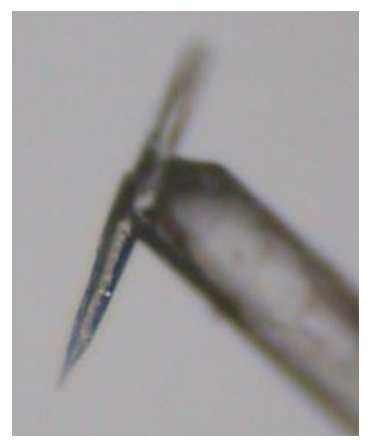

Figure S-4. Sample of 5e employed for the X-ray analysis, mounted on the top of a capillary fibre with a drop of perfluorinated oil. 
The experiment was carried out at room temperature and explored a $99.6 \%$ complete sphere of data up to $53.2 \mathrm{deg}$ of $2 \theta$. The dataset consisted of 14223 reflections from both twins, of which 8405 were intense $(\mathrm{I}>2 \sigma(\mathrm{I}))$. Data reduction, structure solution and refinement were carried out with the same techniques and programs detailed for the compound $\mathbf{2 h}$ (see above), but an explicit treatment of twinning was here applied. The two coexisting lattices were rotated by $\sim 5^{\circ}$ around the [010] direction with respect to each other. The analysis of the diffraction intensities quantified their relative weights as $76 \%$ and $24 \%$. In any case, only a minority of the recorded intensities (31 reflections, 24 unique) were contaminated by twins overlap. Therefore, we first solved the structure against a HKLF 4 set of merged data from the main lattice. Then, a more accurate BASF refinement was carried out against a HKLF 5 unmerged dataset, which included reflections of both the twin individuals.

The compound 5e crystallizes in the monoclinic, acentric and polar $P 2_{1}$ space group $\left(\mathrm{n}^{\mathrm{o}} 4\right)$ with one molecule per asymmetric unit. Figure S5 shows the in-crystal molecular conformation, as well as the absolute configuration of the two chiral centres, which were unequivocally determined as $\mathrm{R}$ (C2) and R (C20) due to the presence of an anomalous scatterer (S) in the unit cell. The Flack parameter $^{21}$ refined to $0.11(8)$. The main inertial axes of the two 6-membered aromatic rings both lie roughly perpendicular to the $\mathrm{C}=\mathrm{O}$ carbonyl in the central imidazolidinone ring. At the same time, the main least-squares phenyl planes are tilted by 58.1(3) deg with respect to each other.

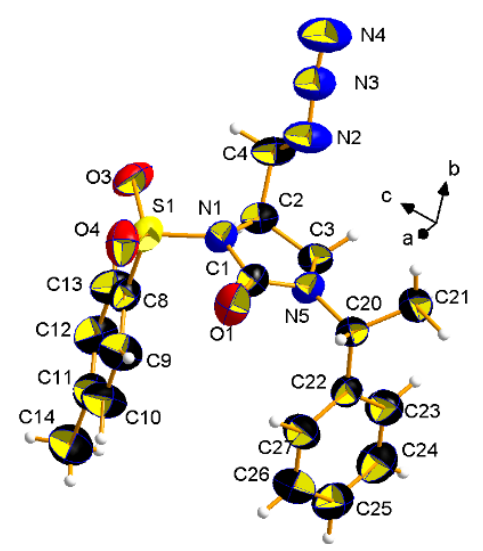

Figure S-5. Asymmetric unit of 5e at RT, with the atom-numbering scheme and the crystallographic reference system highlighted. Thermal ellipsoids of non-H atoms were drawn at the $50 \%$ probability level. The usual colour code was employed to draw atoms (black: C; white: H; red: O; blue: N; yellow: S).

[21] Parsons, S.; Flack, H. D.; Wagner, T., Acta Cryst. 2013, B69, 249-259 
The covalent $\mathrm{N}-\mathrm{N}$ bonds in the azide group are essentially identical with those measured for $\mathbf{2 h}$ (see above), with $\mathrm{d}_{\mathrm{N} 2-\mathrm{N} 3}=1.22(1) \AA$ and $\mathrm{d}_{\mathrm{N} 3-\mathrm{N} 4}=1.13(1) \AA$.

Unit cell: $\mathrm{a}=6.3060(7) \AA, \mathrm{b}=16.9358(18) \AA, \mathrm{c}=9.8132(10) \AA, \beta=107.276(6) \operatorname{deg}, \mathrm{V}=$ 1000.74(19) $\AA^{3}$, density: $1.326 \mathrm{~g} / \mathrm{cm}^{3}$. The final least squares model refined to an agreement factor $R_{1}(F)$ of 0.0593 for $8405 F_{\mathrm{o}}>4 \sigma\left(F_{\mathrm{o}}\right)$, which became 0.1140 for all the 14223 data. The goodnessof-fit was as large as 0.941 . The Fourier residuals were also reasonably low $\left(\Delta \rho_{\text {MAX/MIN }}=+0.17 /-\right.$ $0.25 \mathrm{e} / \AA^{3}$ ). CCDC 1970339 contains the supplementary crystallographic data for this paper. These data can be obtained free of charge from The Cambridge Crystallographic Data Centre via www.ccdc.cam.ac.uk/structures.

Figure S6 shows the crystal packing of 5e. Azide chains align all in the same direction along the monoclinic $b$ axis, and are oppositely oriented with respect to the tosyl group (Figure $2 \mathrm{a}$ ). On the contrary, $\mathrm{C}=\mathrm{O}$ functions on 5-membered rings are arranged antiparallel to each other along the perpendicular $a$ direction (Figure $2 \mathrm{~b}$ ). No strong hydrogen bond donors are present; moreover, the mutual orientation of screw-related molecules prevents the formation of short intermolecular $\mathrm{CH} \cdots \mathrm{O}$ contacts and only a minority of weak intramolecular $\mathrm{CH} \cdots \mathrm{O}$ and $\mathrm{CH} \cdots \mathrm{N}$ contacts is formed.

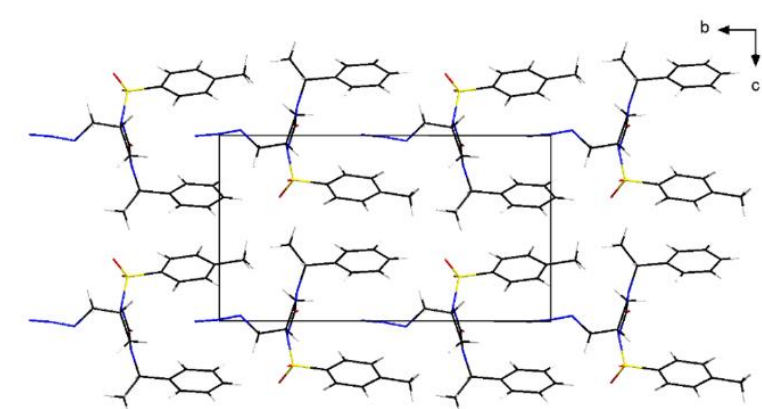

(a)

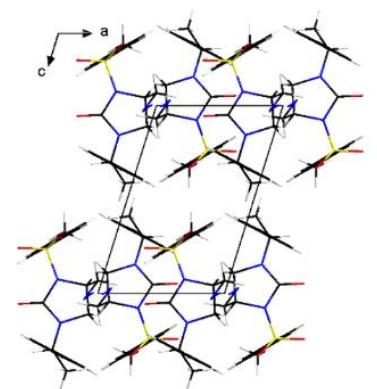

(b)

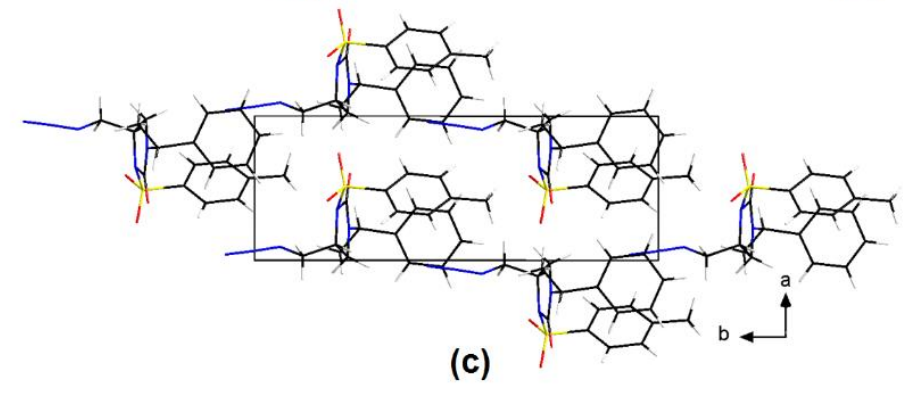

Figure S-6. Wires-stick representation of the crystal packing of 5e, as seen (a) along the $a$ cell axis; (b) the $b$ cell axis; (c) the $c$ cell axis. Colour code as in Figure S5. 
${ }^{1} \mathrm{H}-\mathrm{NMR}$ and ${ }^{13} \mathrm{C}-\mathrm{NMR}$ spectra of unknown compounds

O-Allyl-N-2-nitrophenylsulphonyl-carbamate (1e)

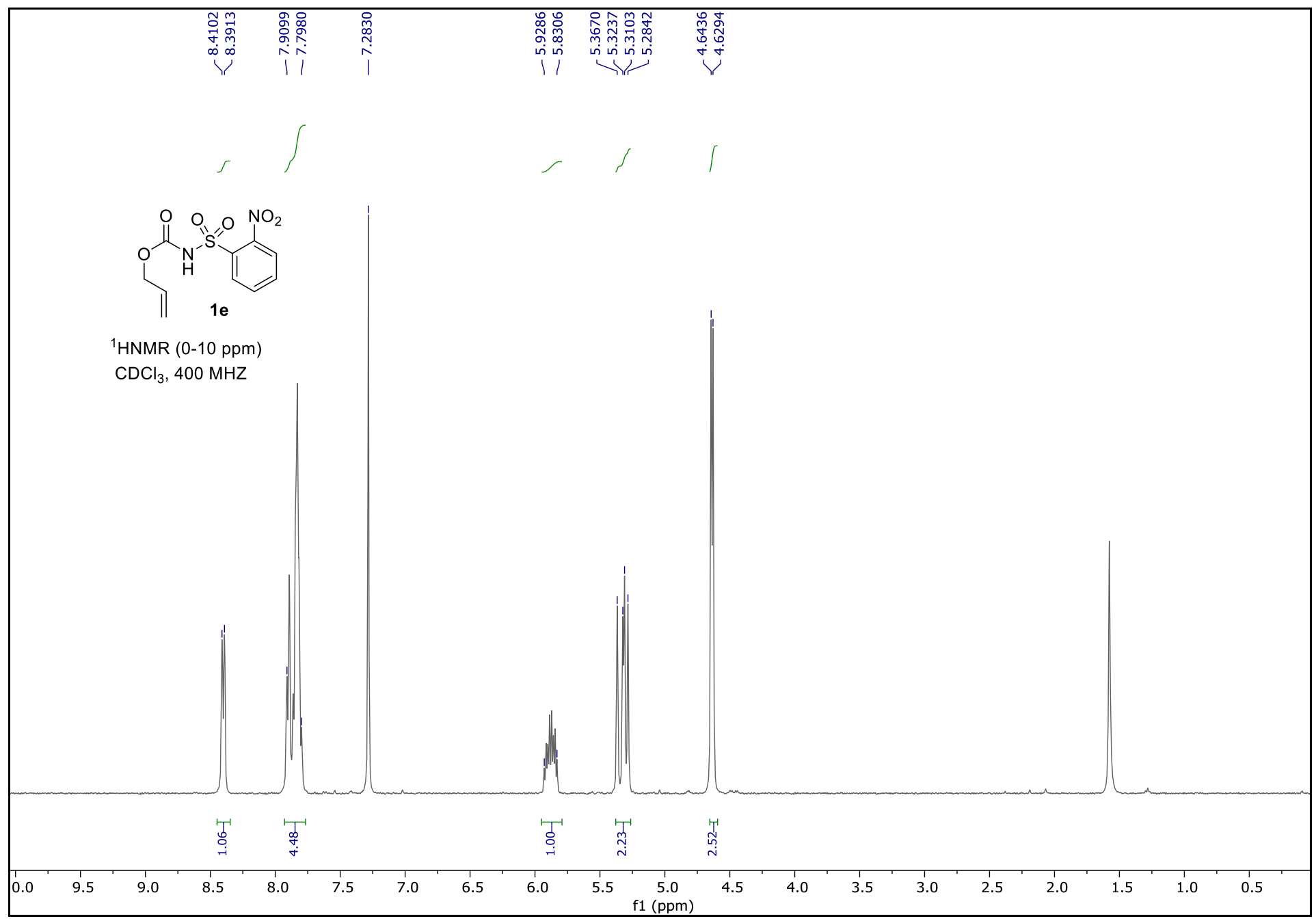

S-31 


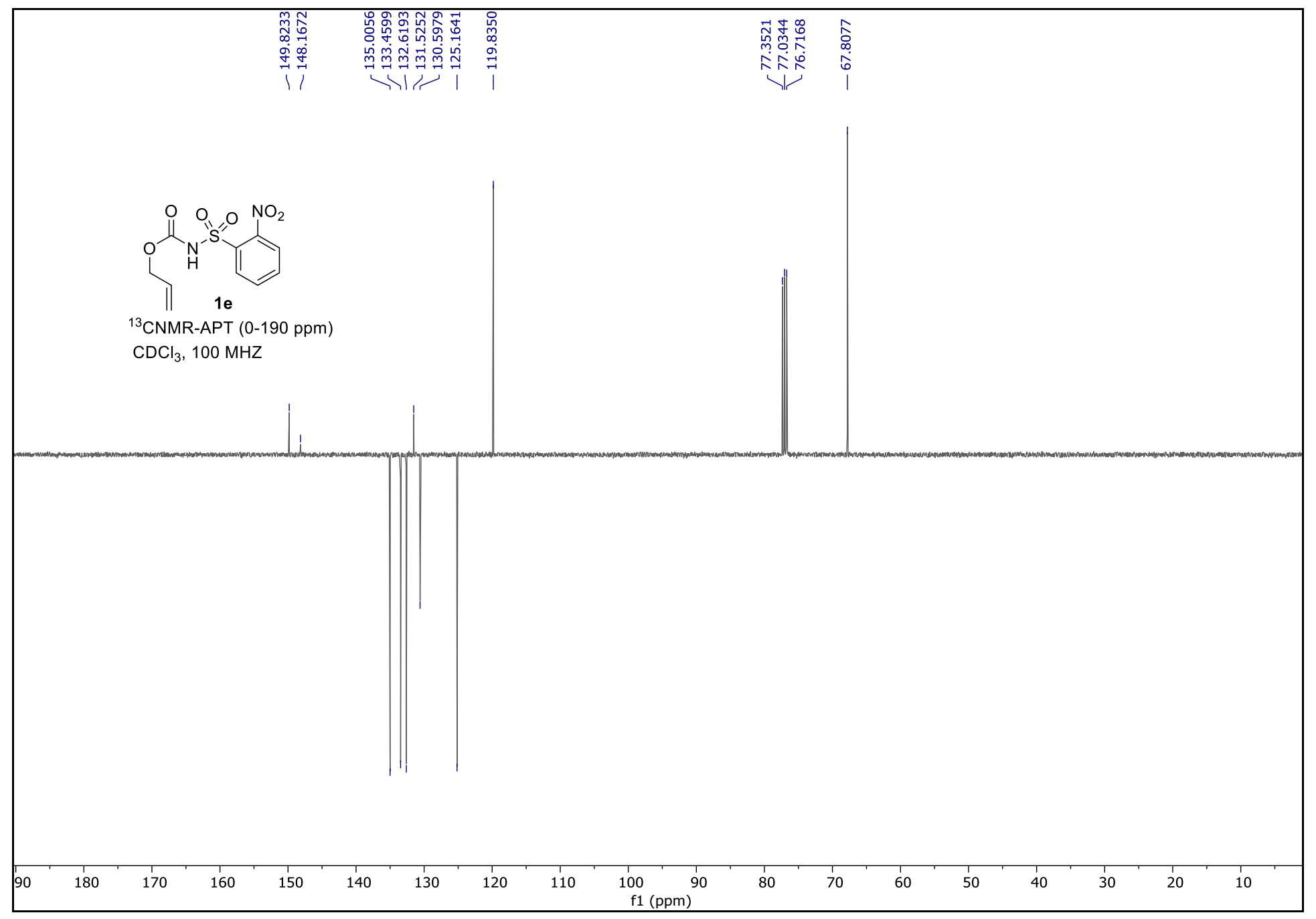




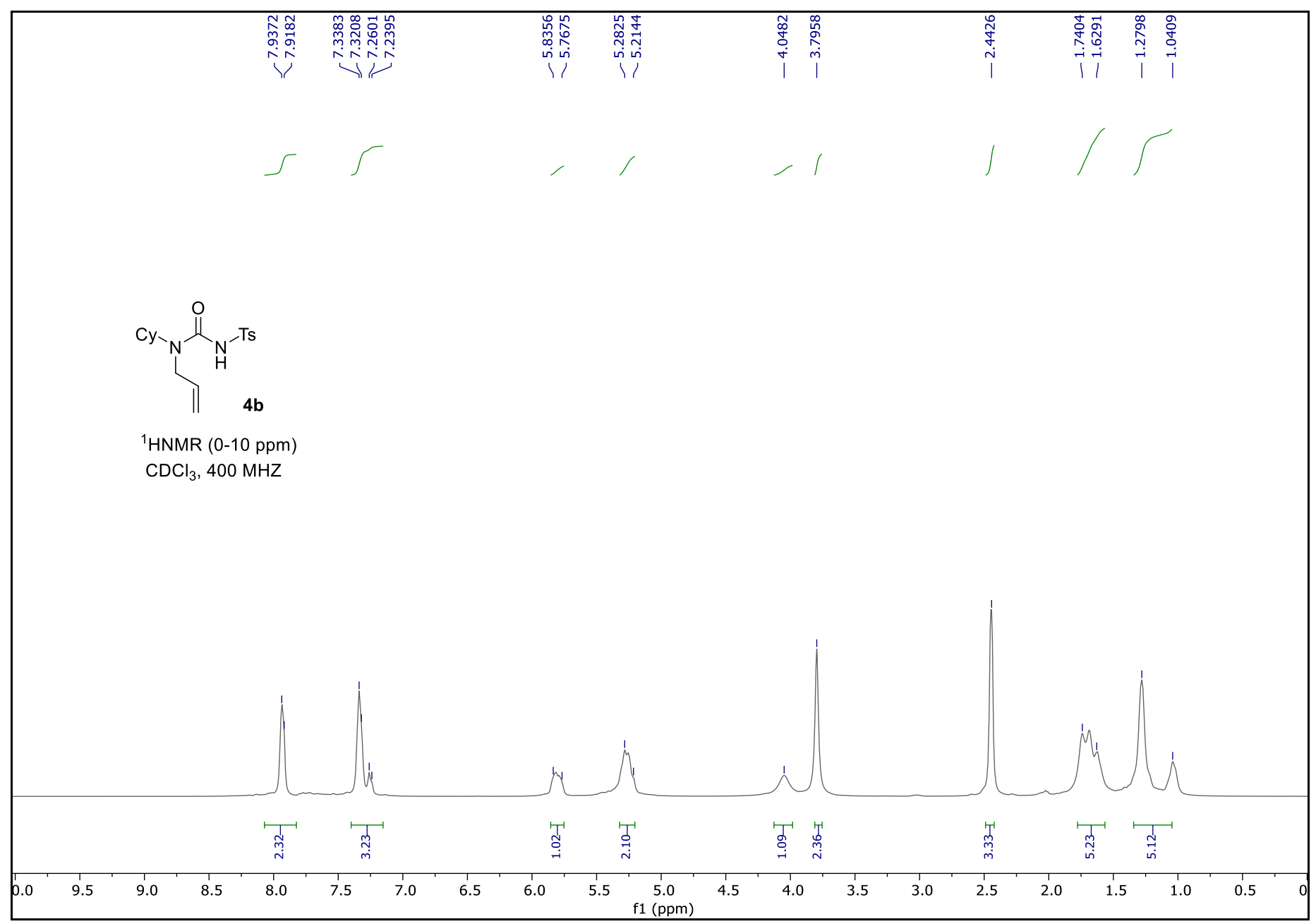




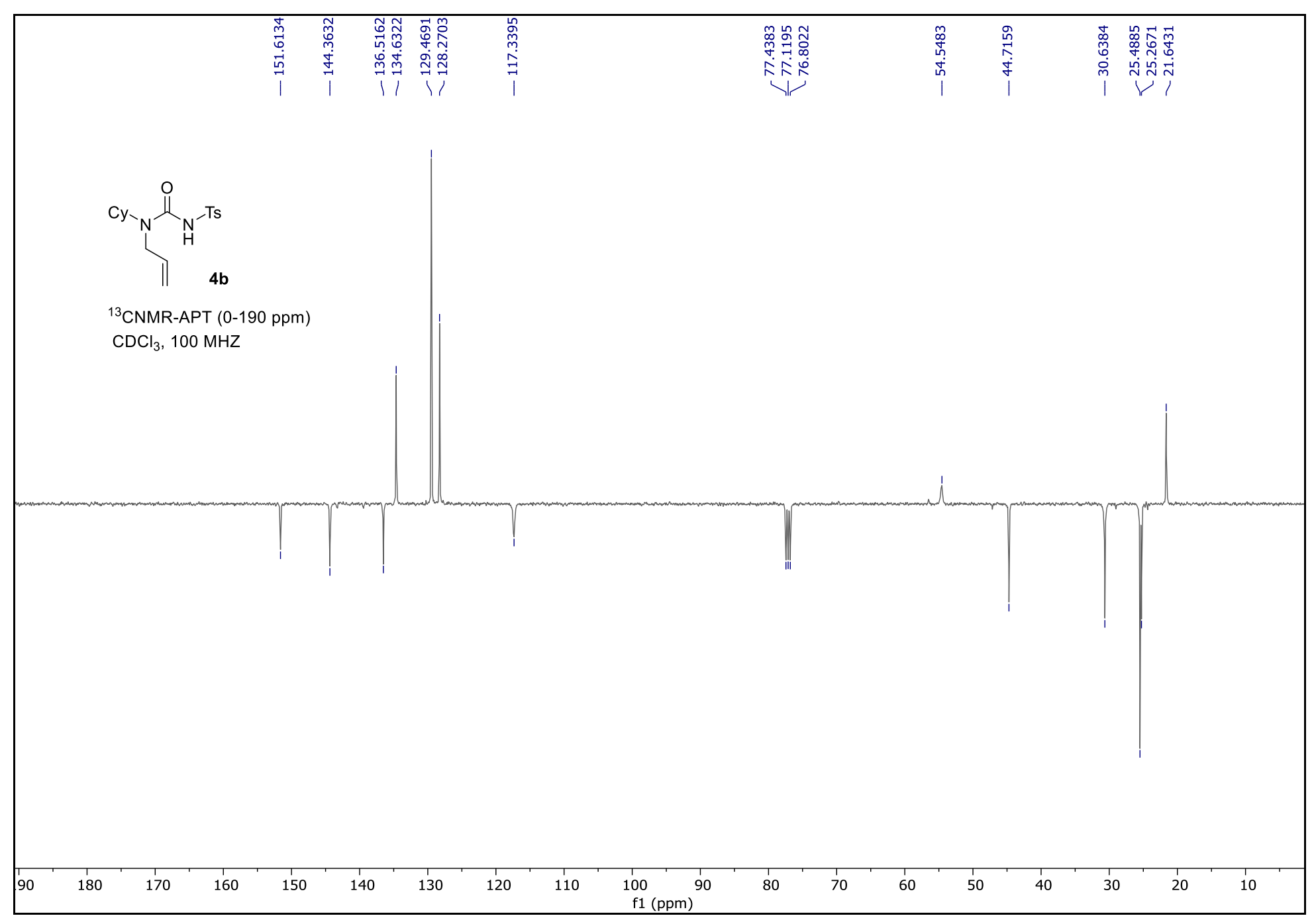


(R)-N-(allyl(1-phenylethyl)carbamoyl)-4-methylbenzenesulfonamide (4d)

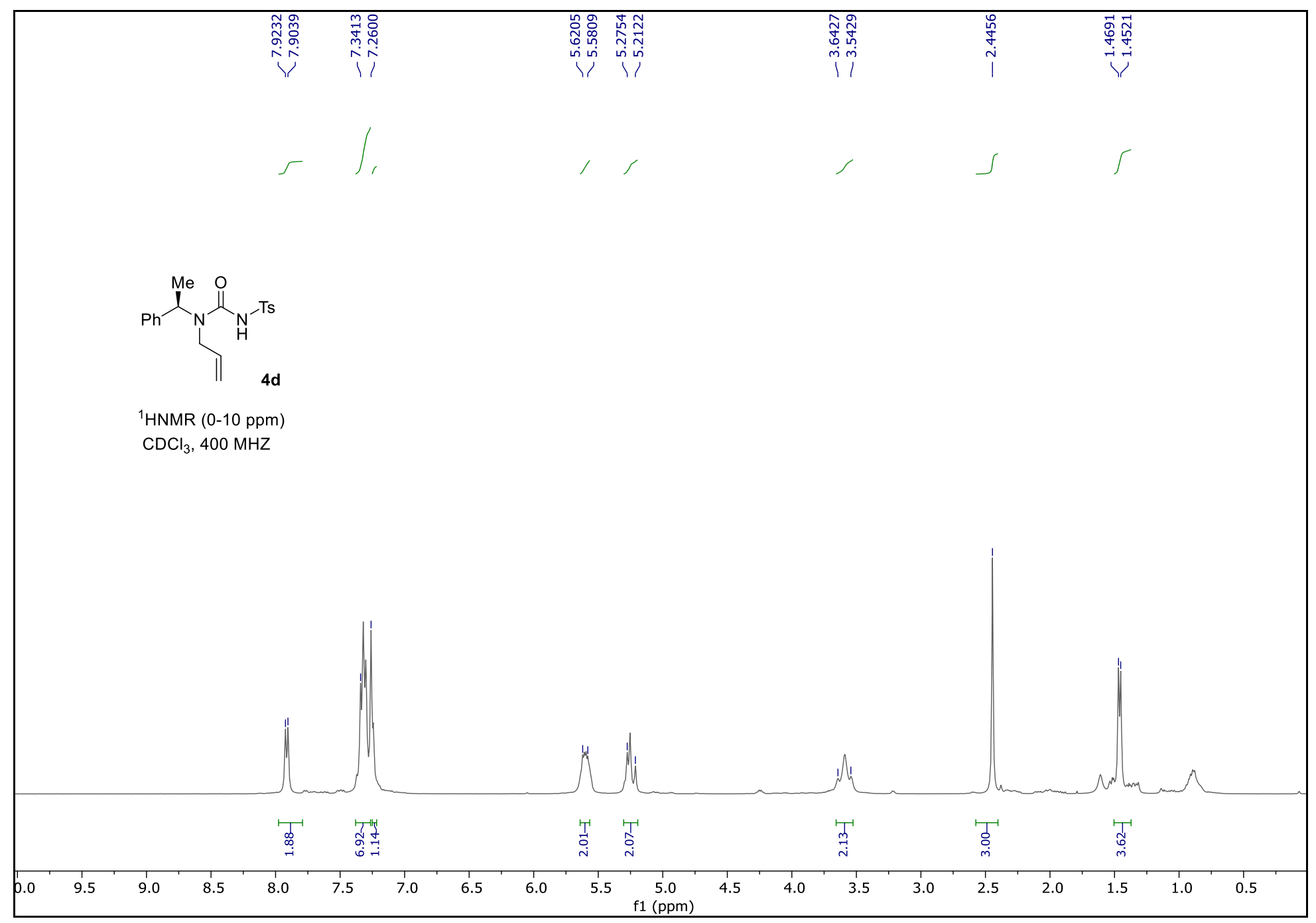




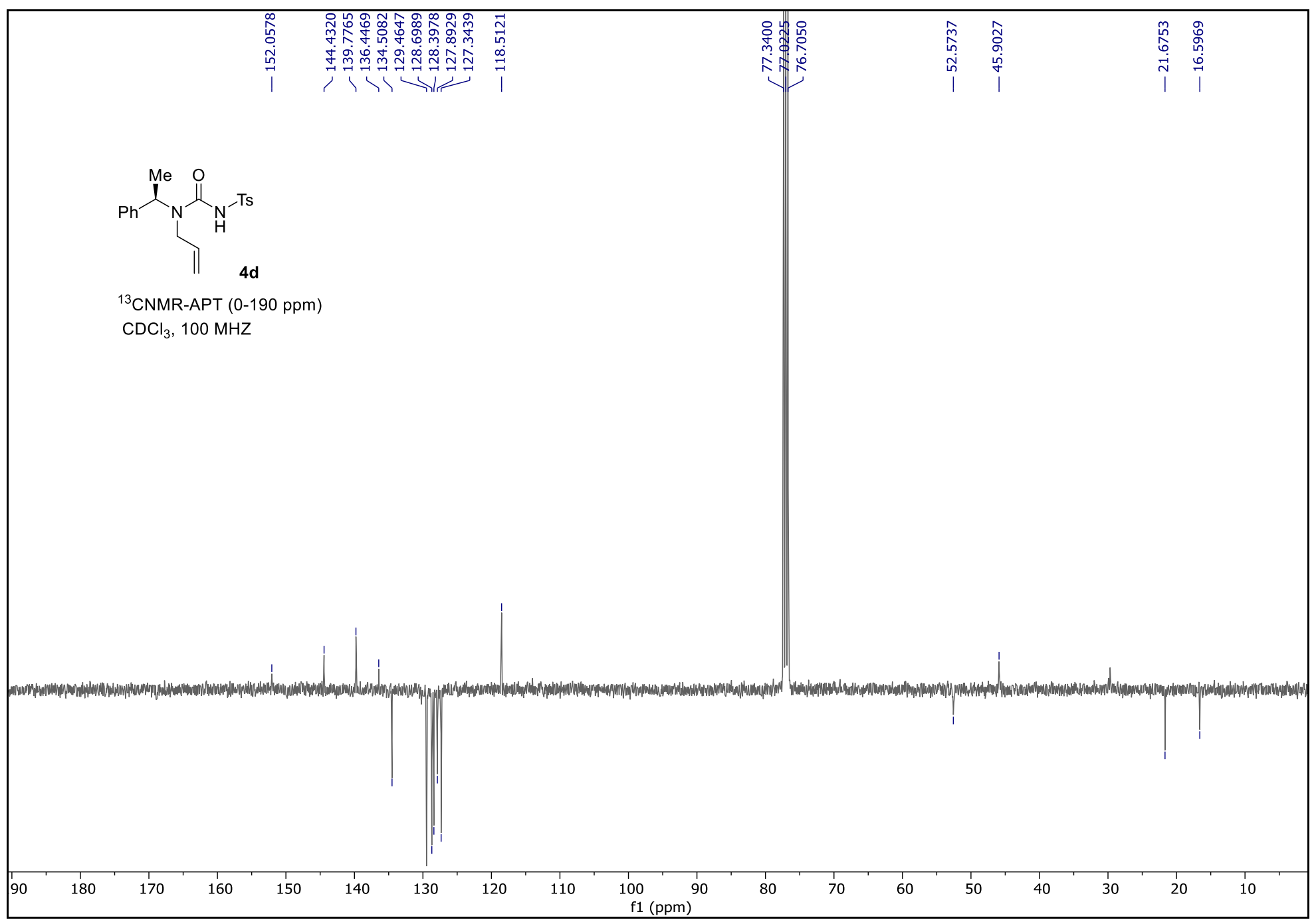




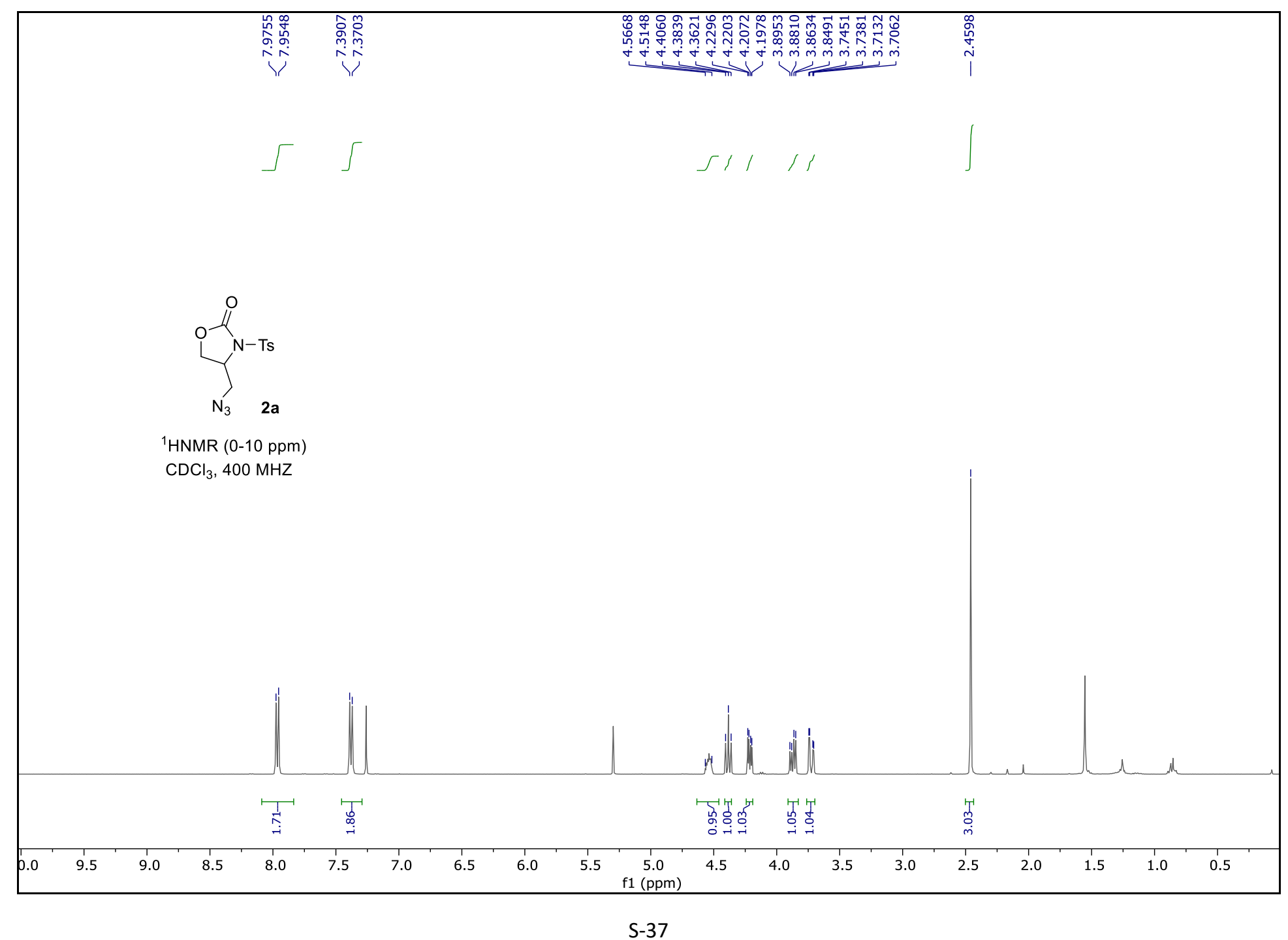




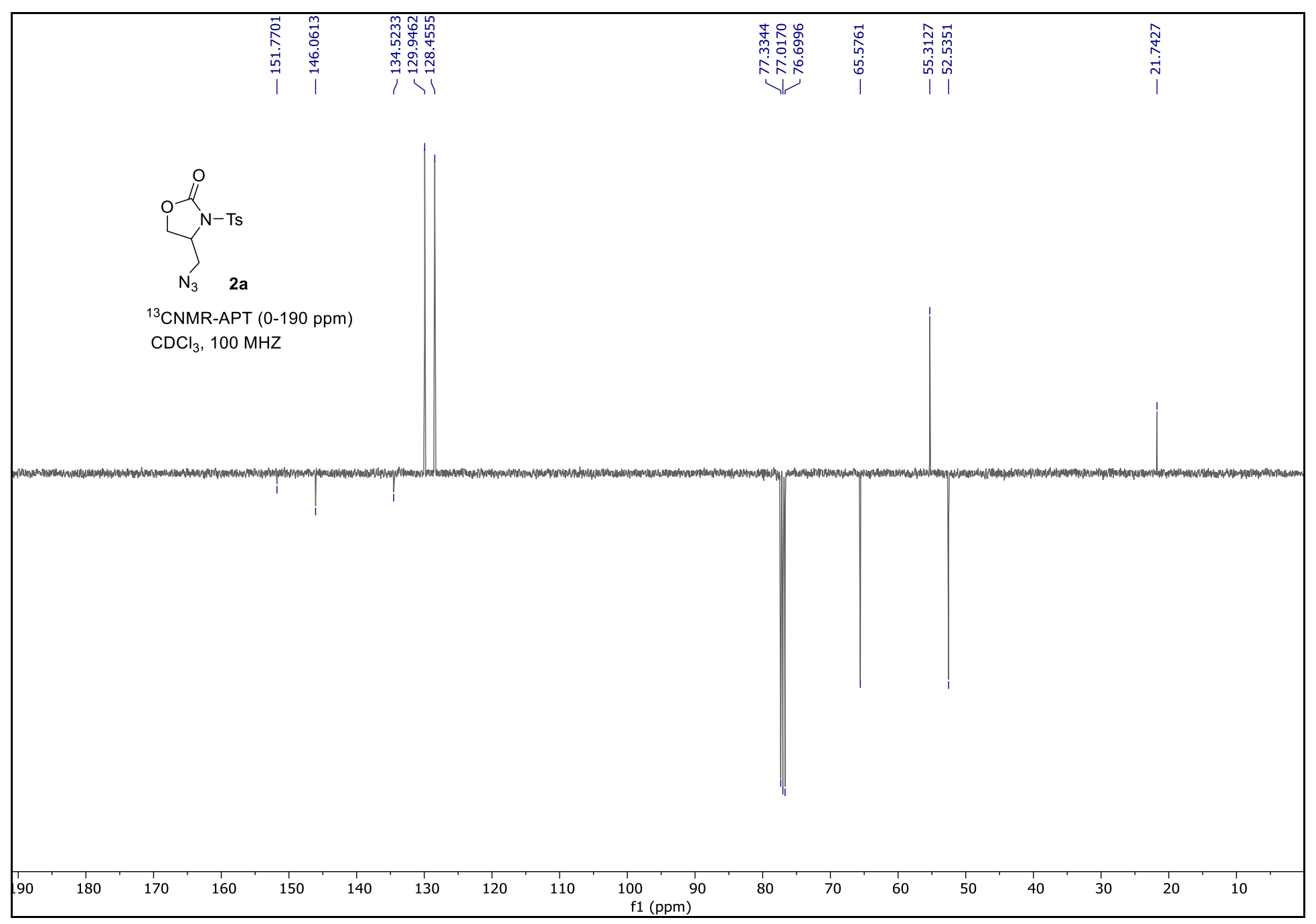


4-(Azidomethyl)-3-(4-nitrophenyl) oxazolidin-2-one (2b)

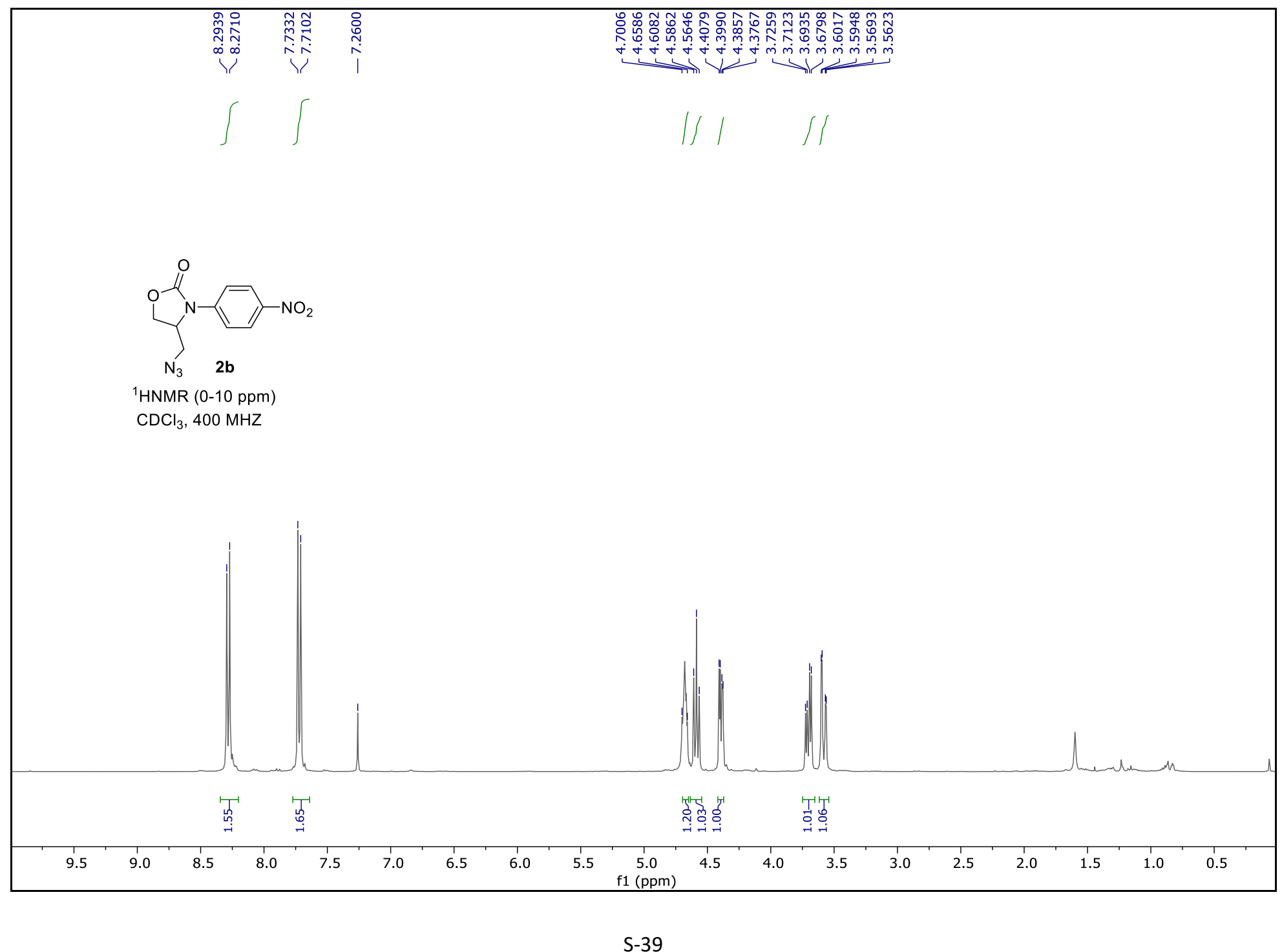




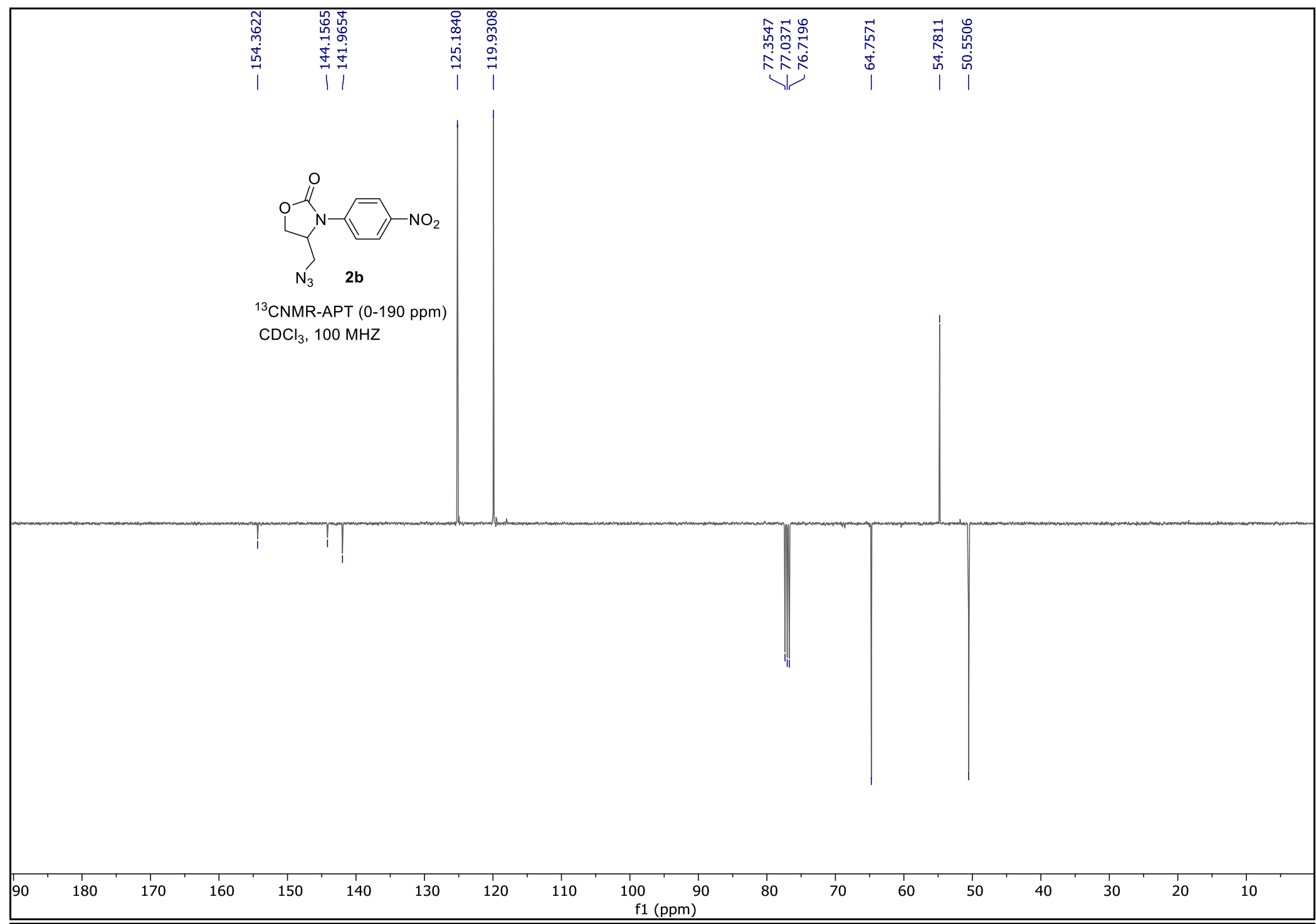


4-(Azidomethyl)-3-[(2-nitrophenyl)sulfonyl]oxazolidin-2-one (2e)

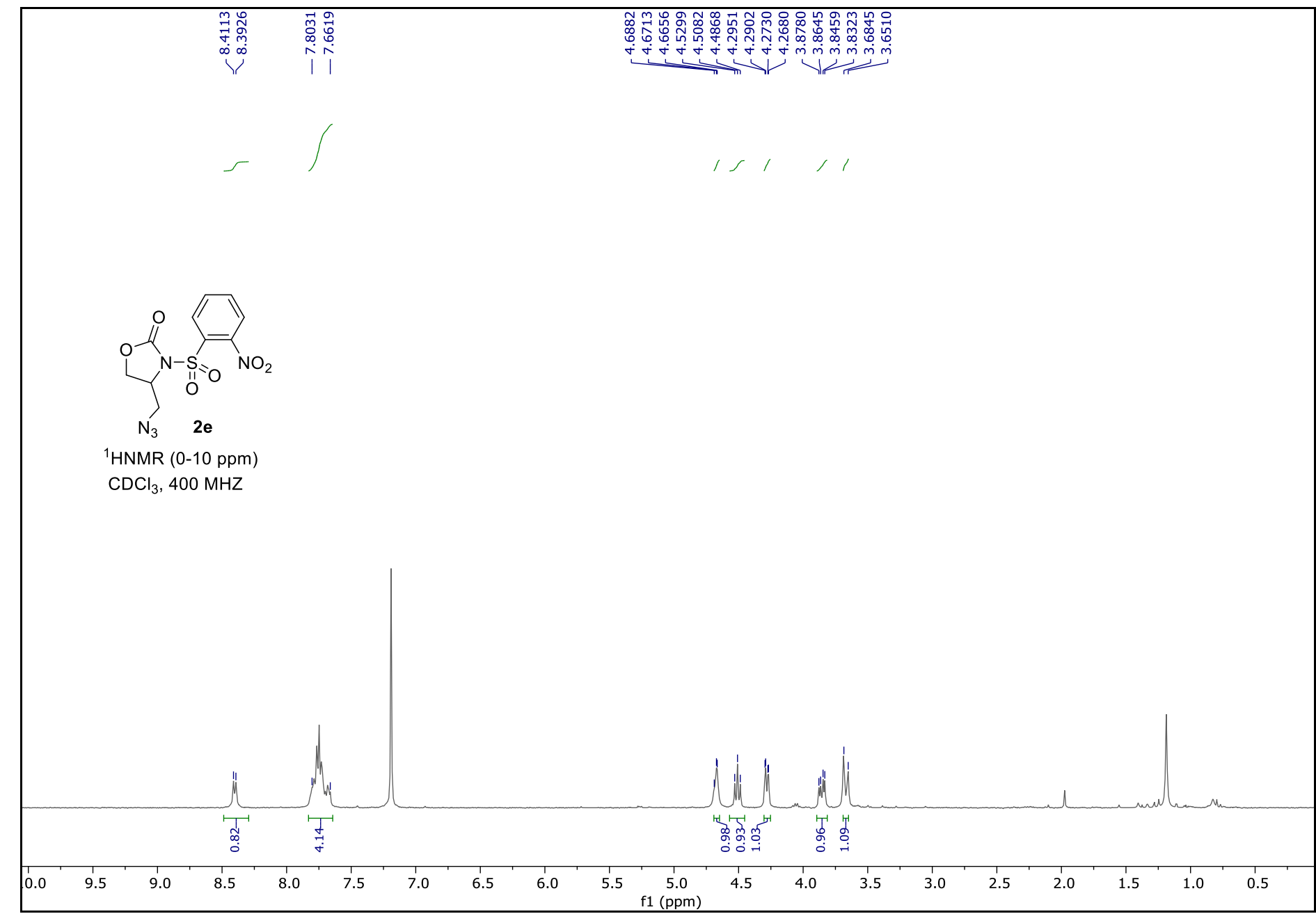




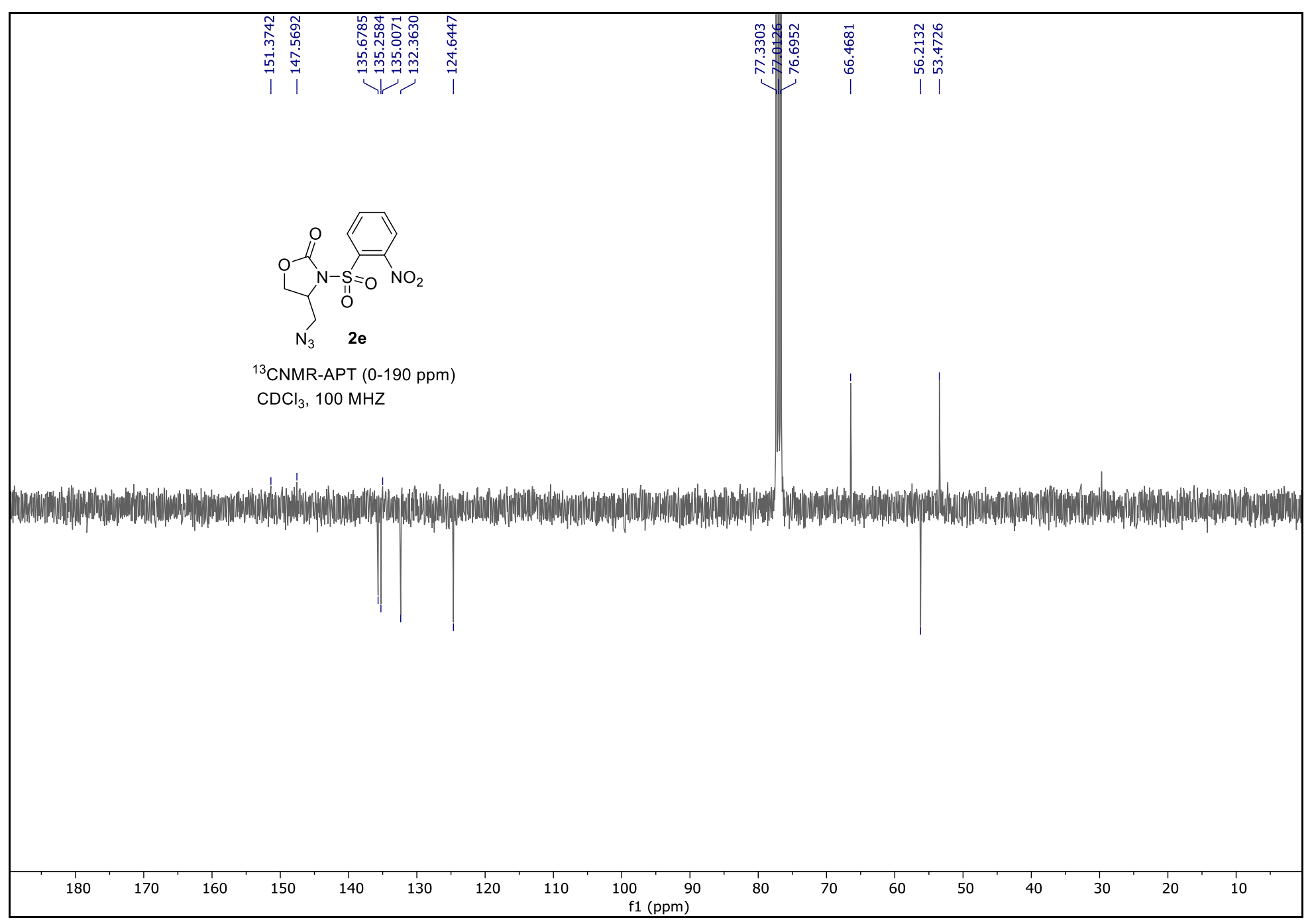


$\left(4 S^{*}, 5 S^{*}\right)-4-($ Azidomethyl)-5-methyl-3-tosyloxazolidin-2-one (2f)

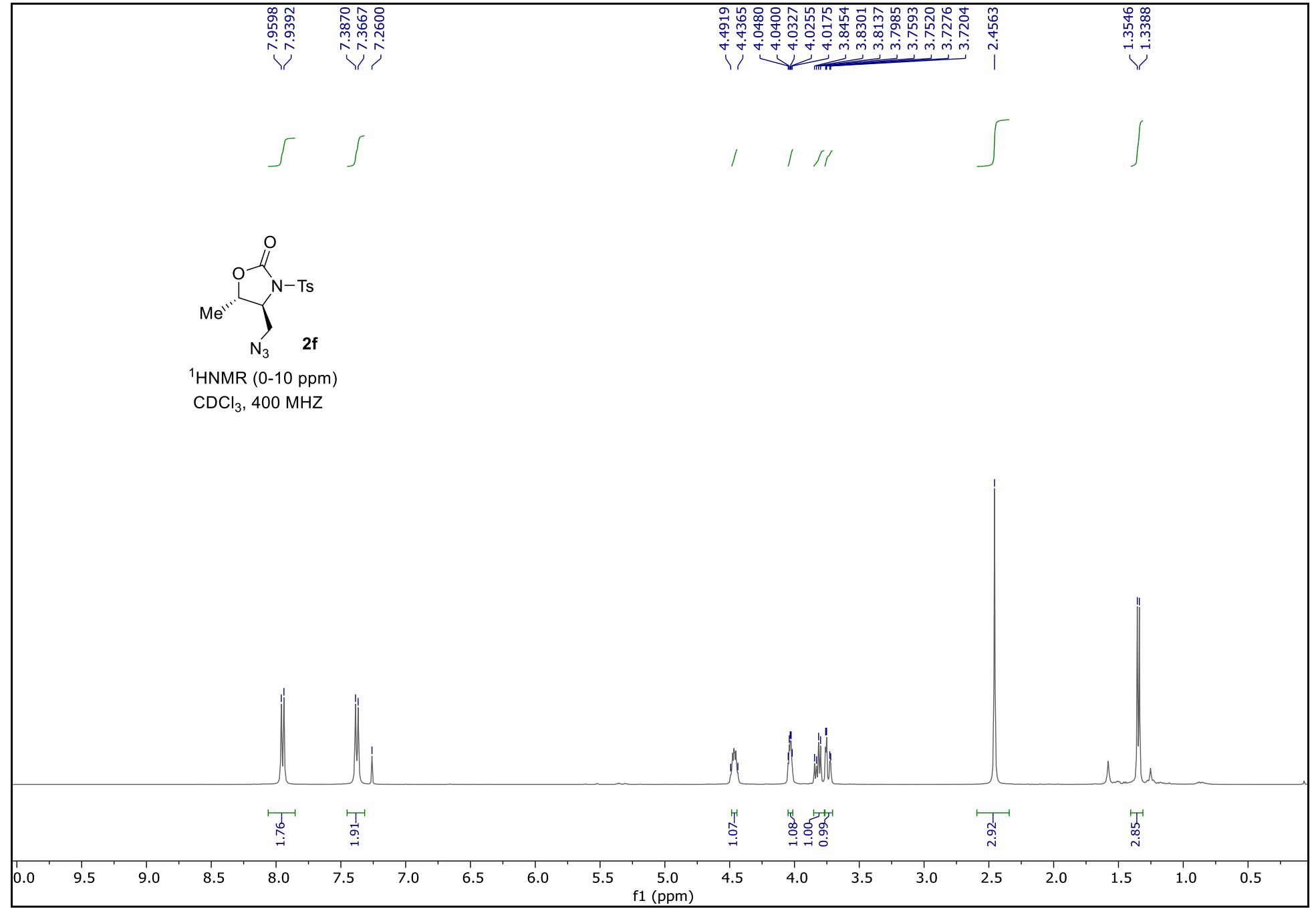




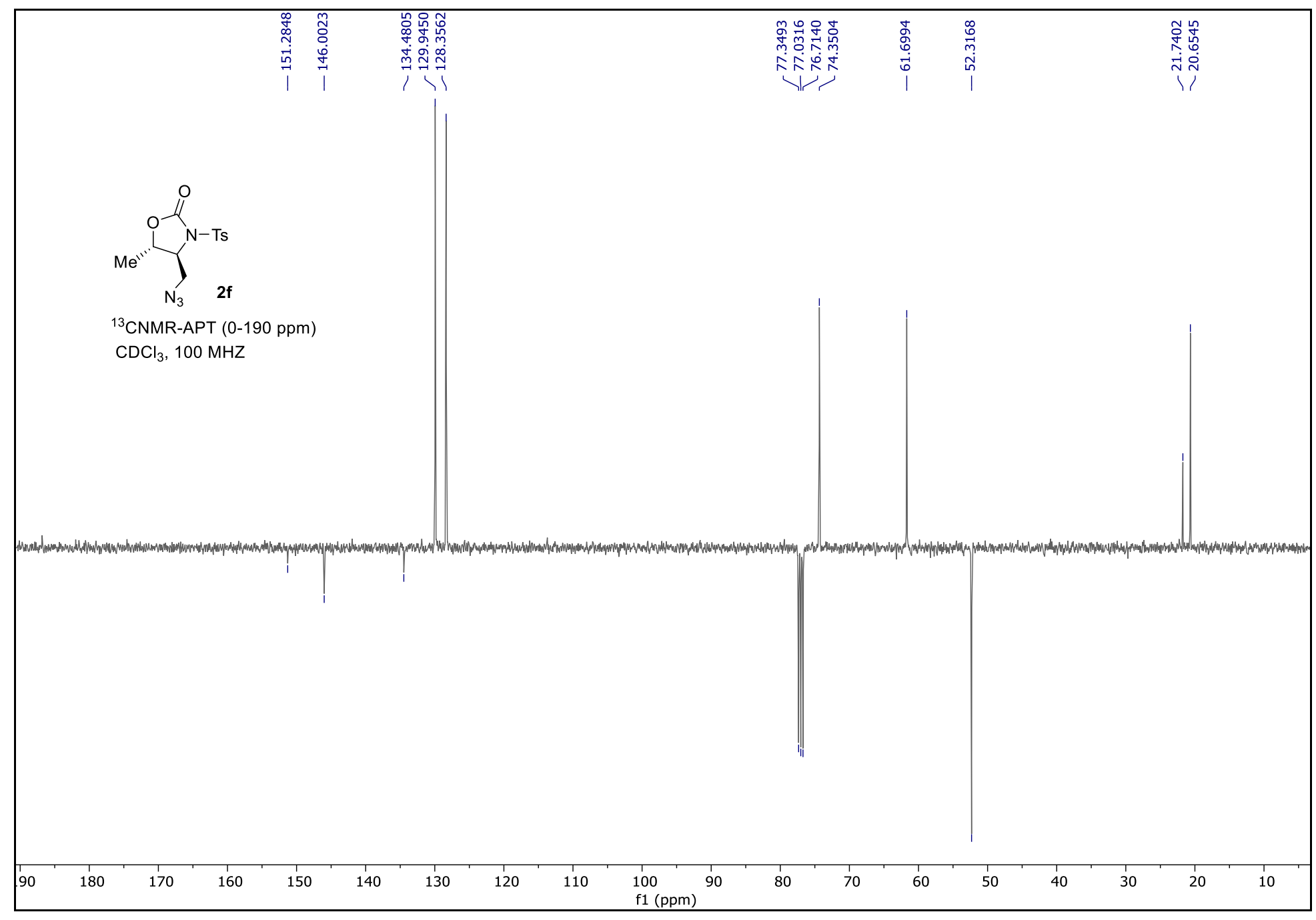


$\left(4 S^{*}, 5 S^{*}\right)-4-($ Azidomethyl)-5-ethyl-3-tosyloxazolidin-2-one (2g)

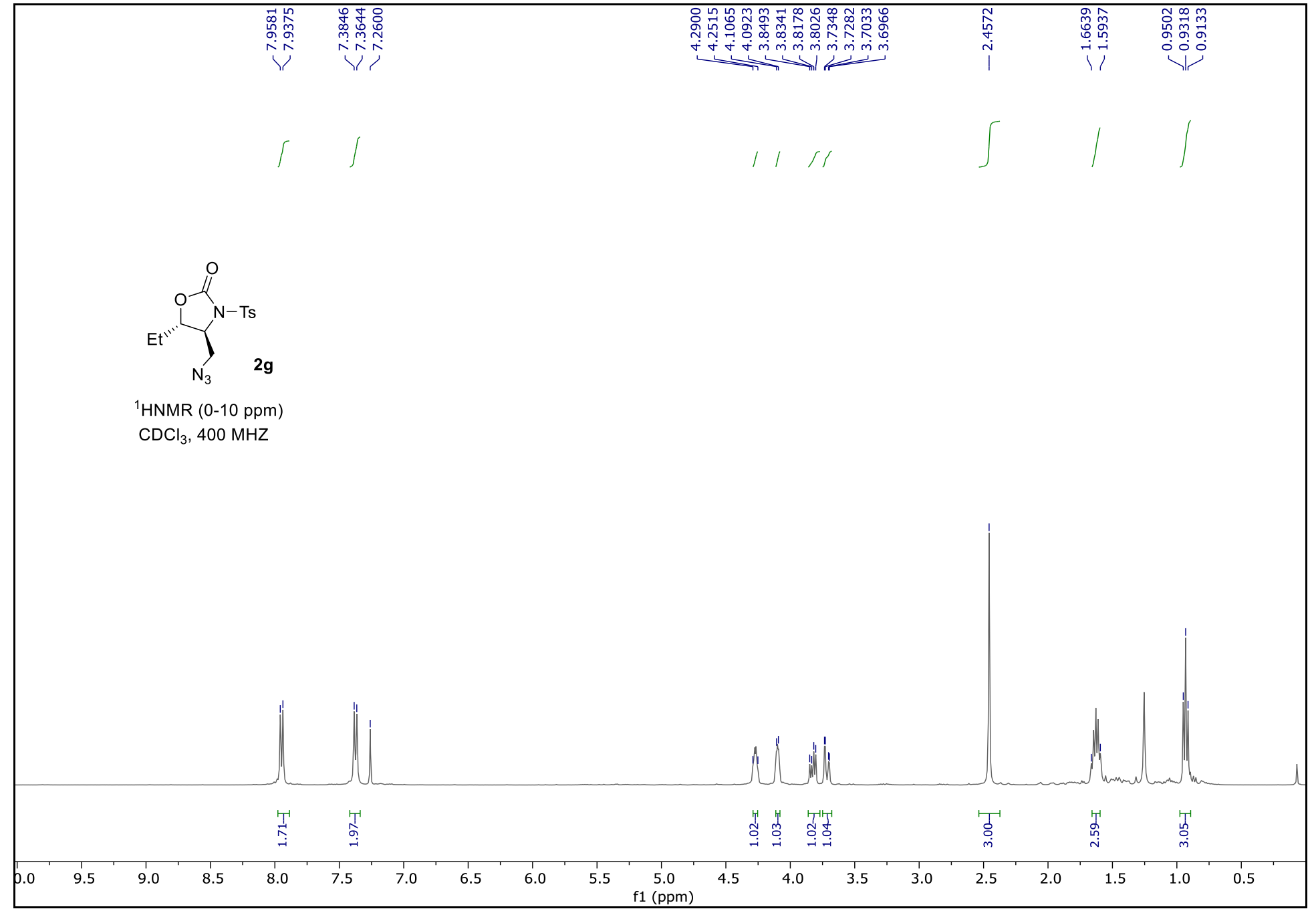




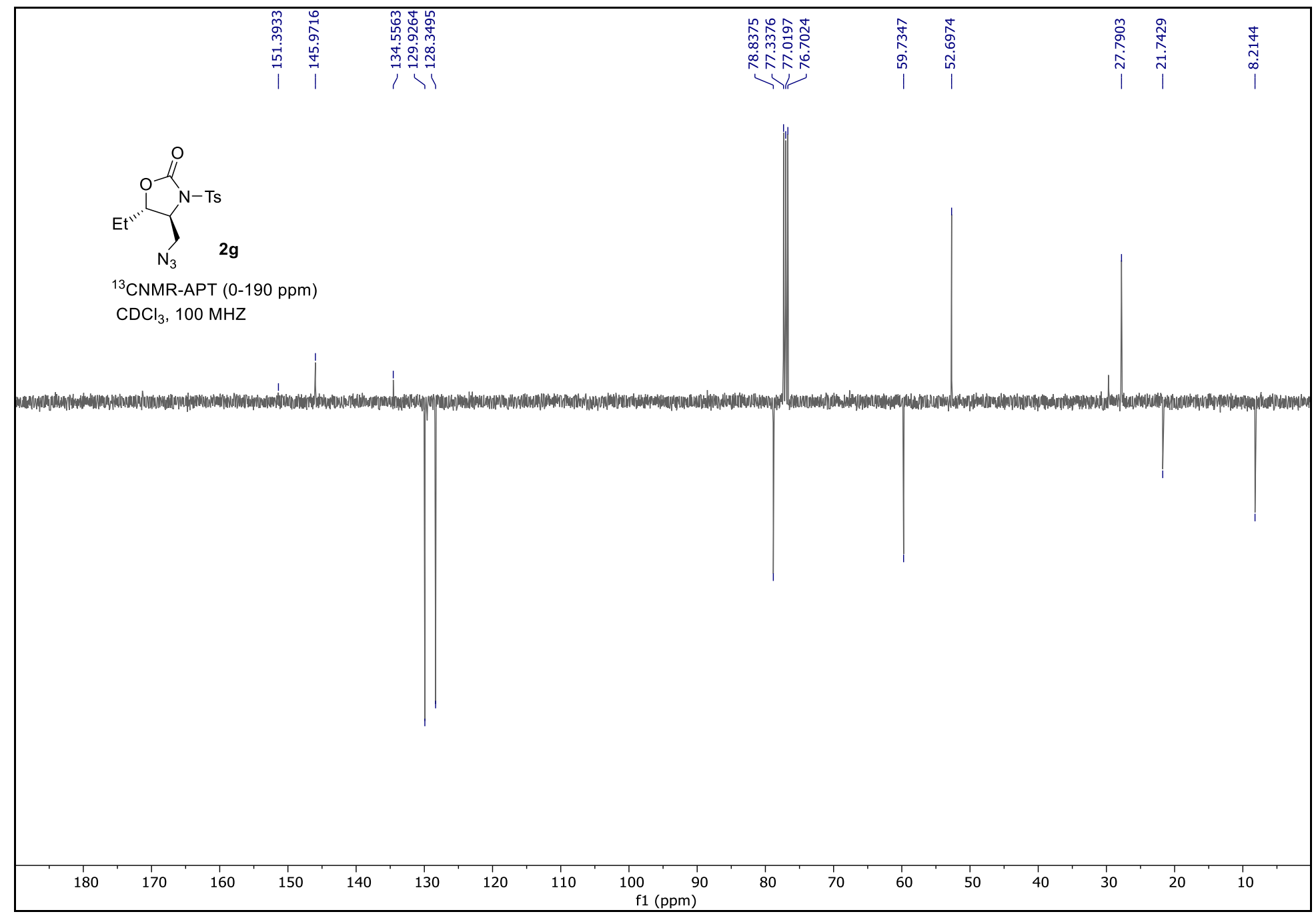

S-46 
(4S*,5S*)-4-(Azidomethyl)-5-propyl-3-tosyloxazolidin-2-one (2h)

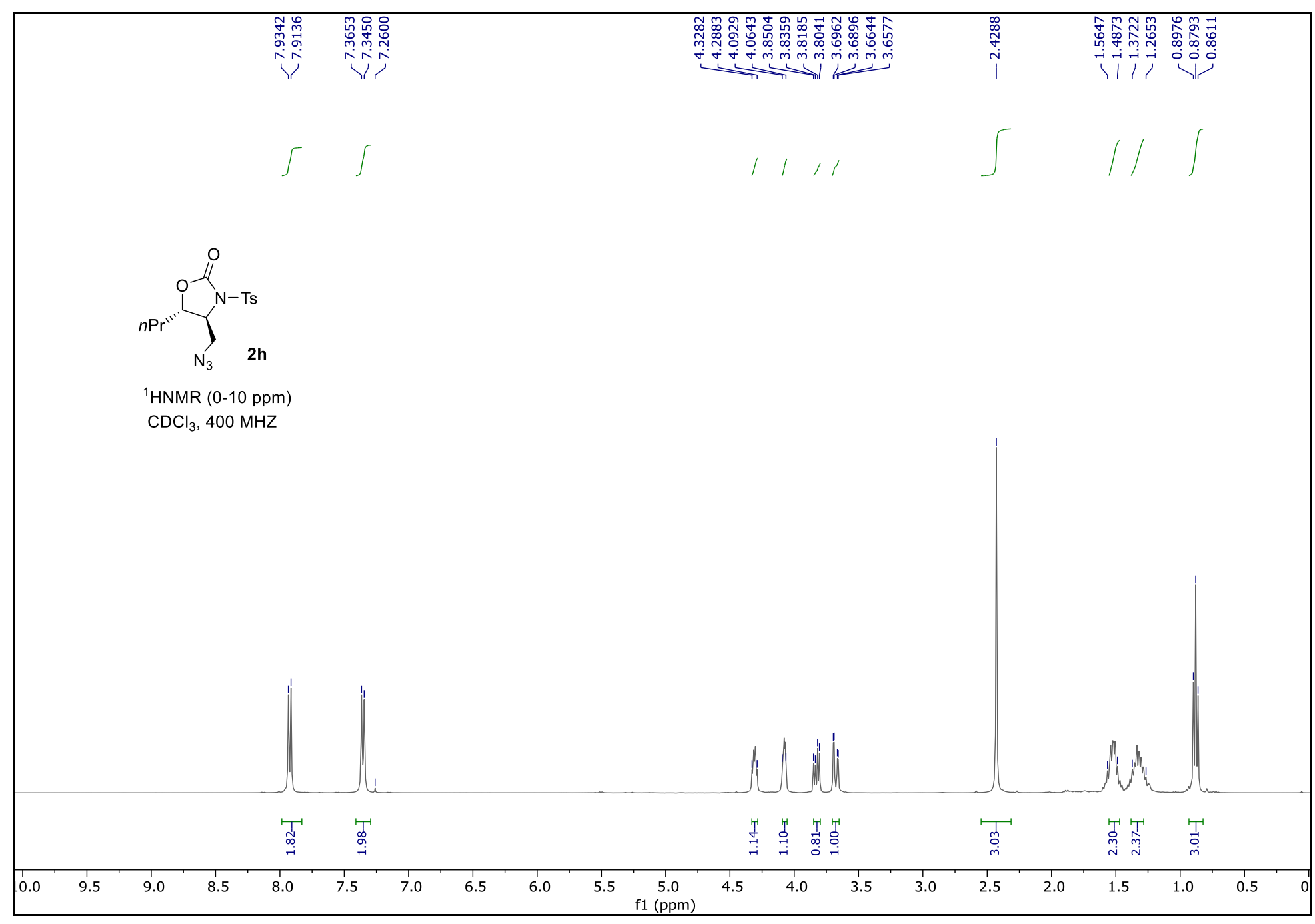




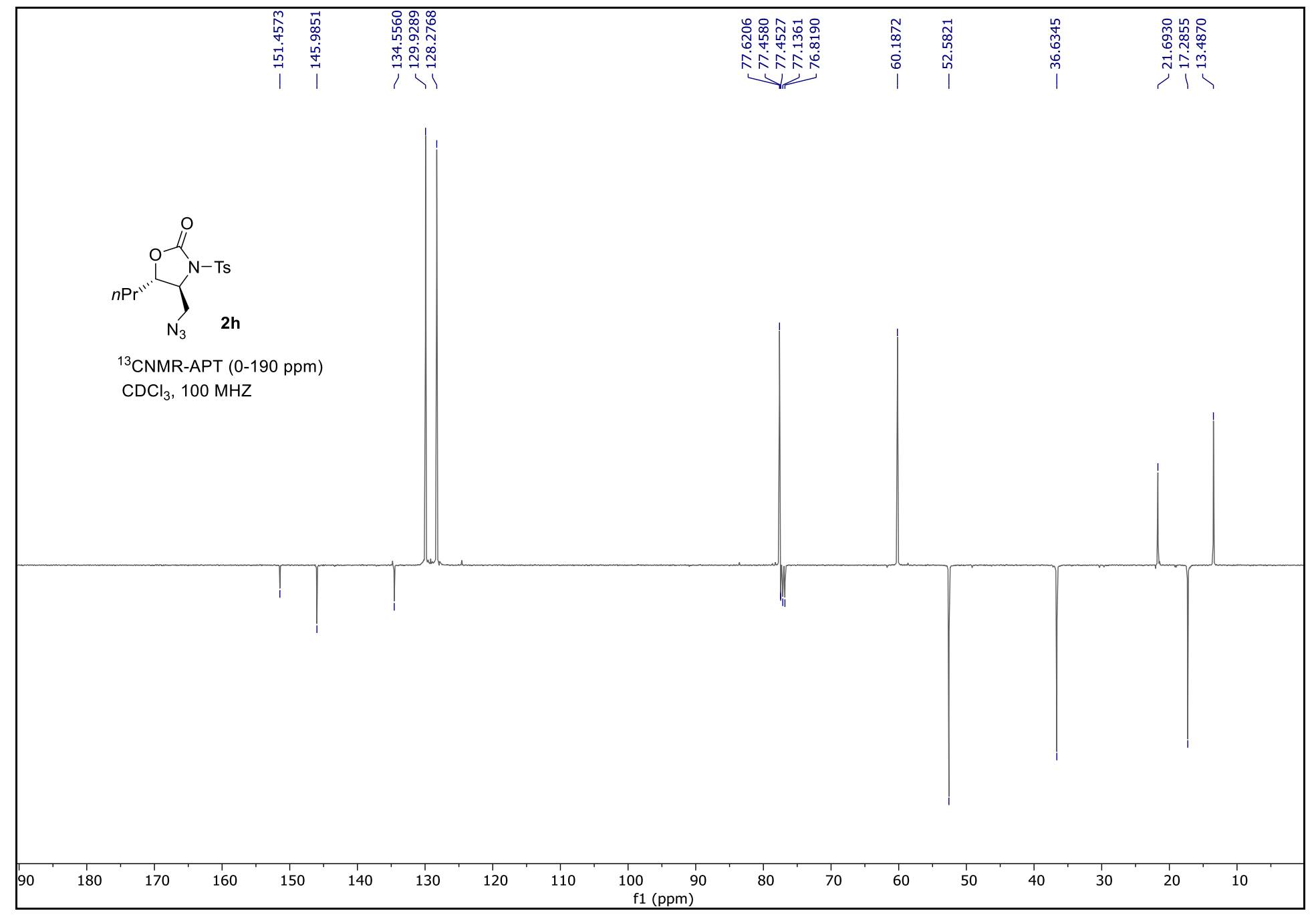


4-(Hydroxymethyl)-5-propyl-3-tosyloxazolidin-2-one (3h)

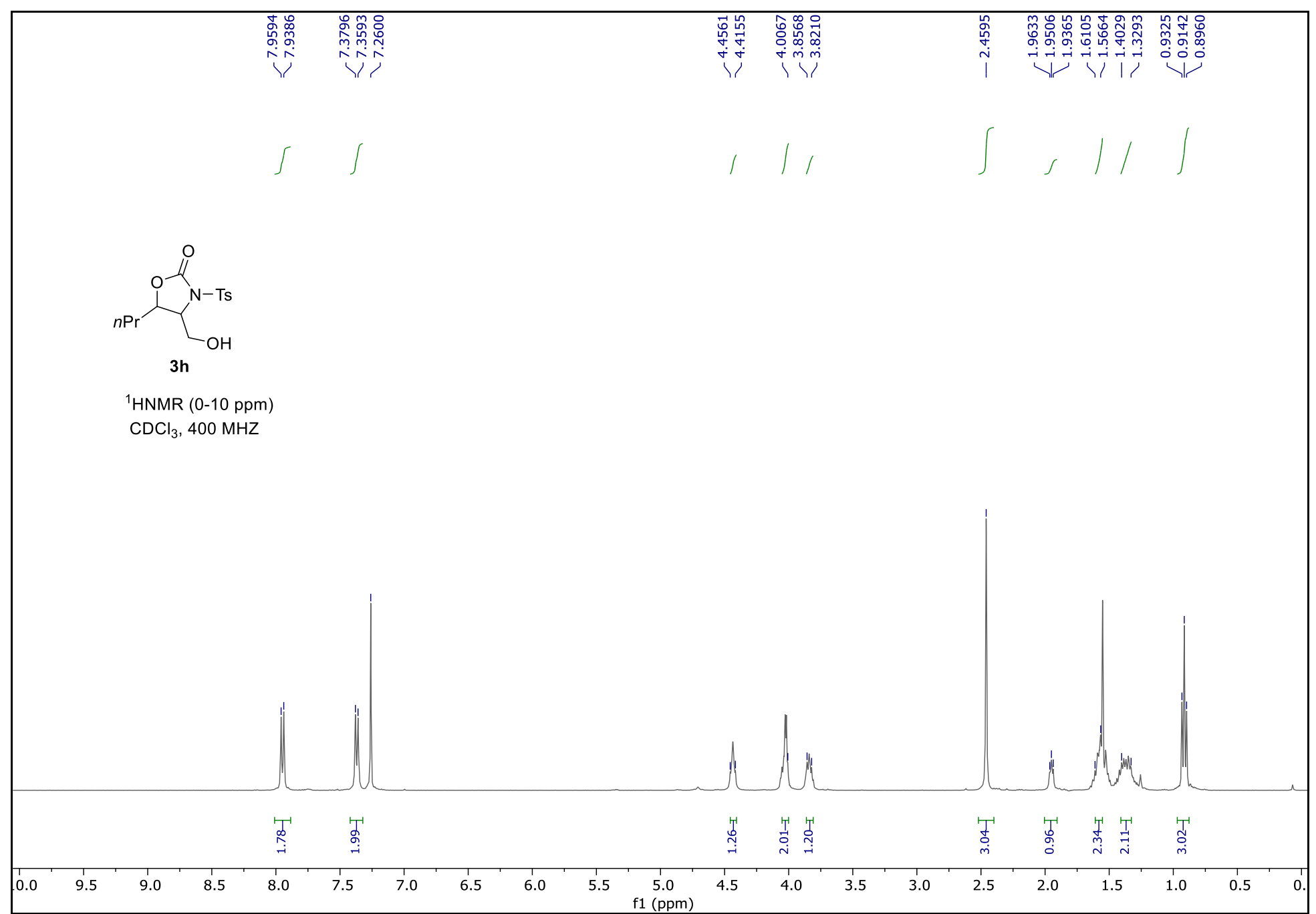




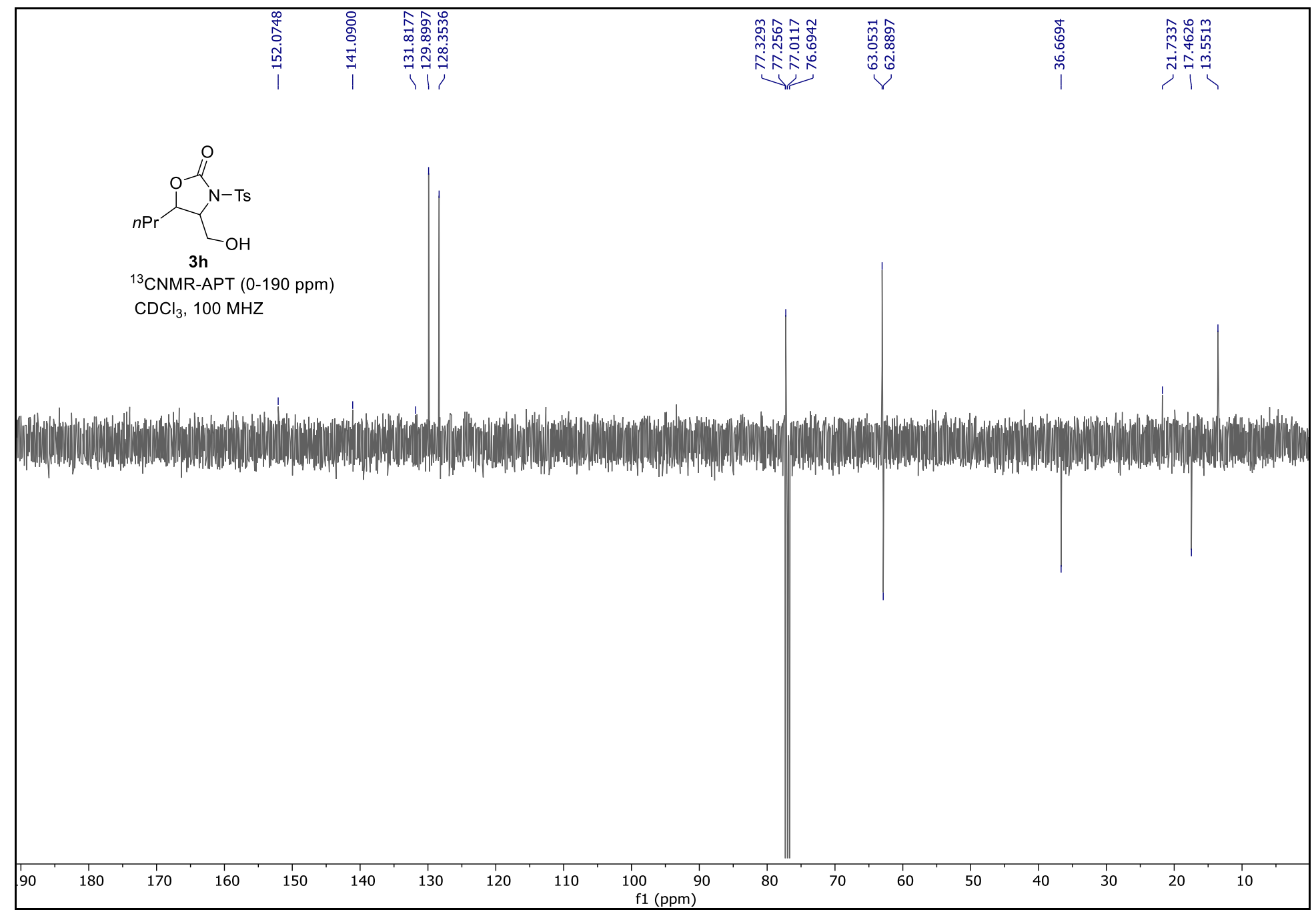


4-(Azidomethyl)-5,5-dimethyl-3-tosyloxazolidin-2-one (2i)

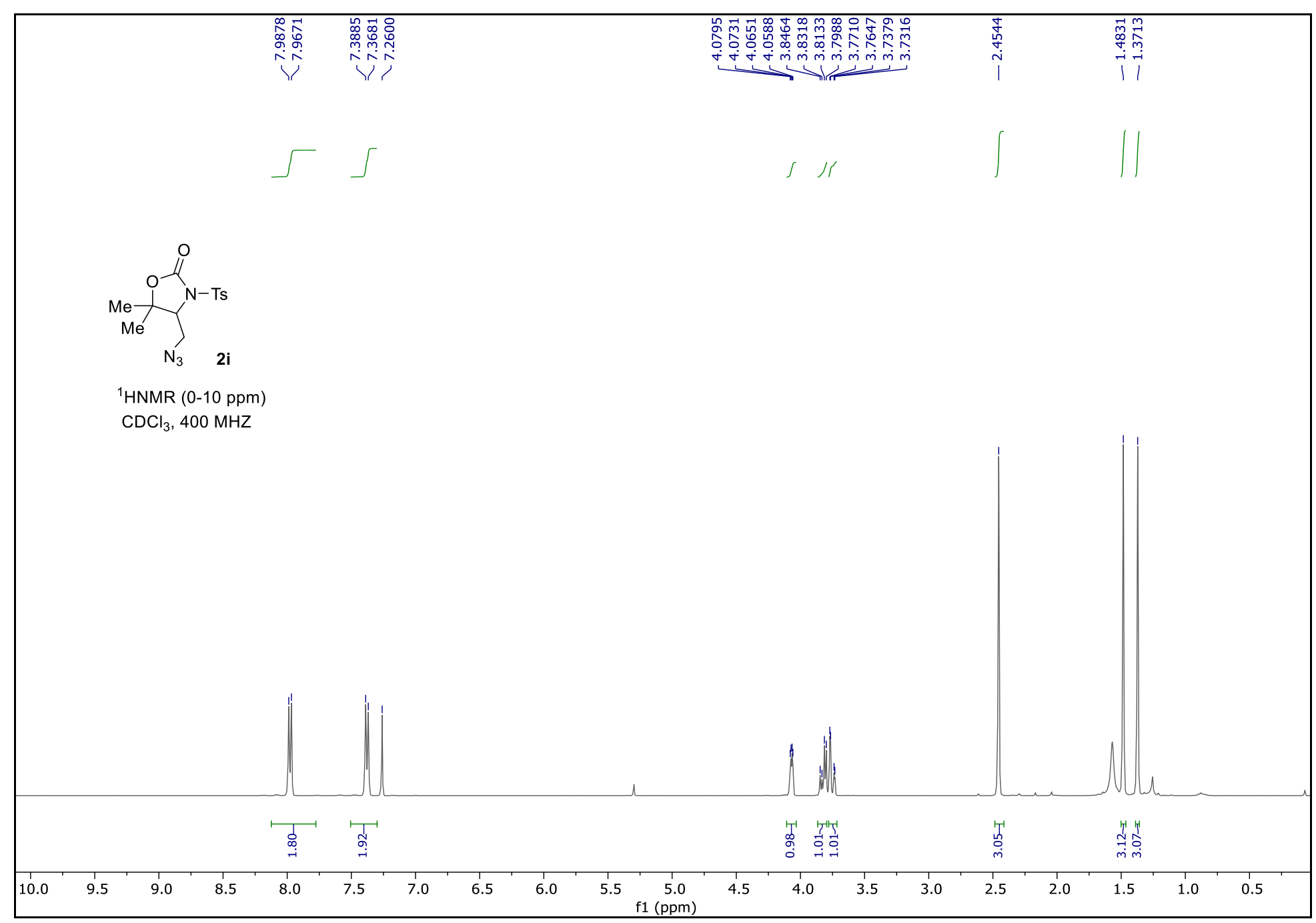




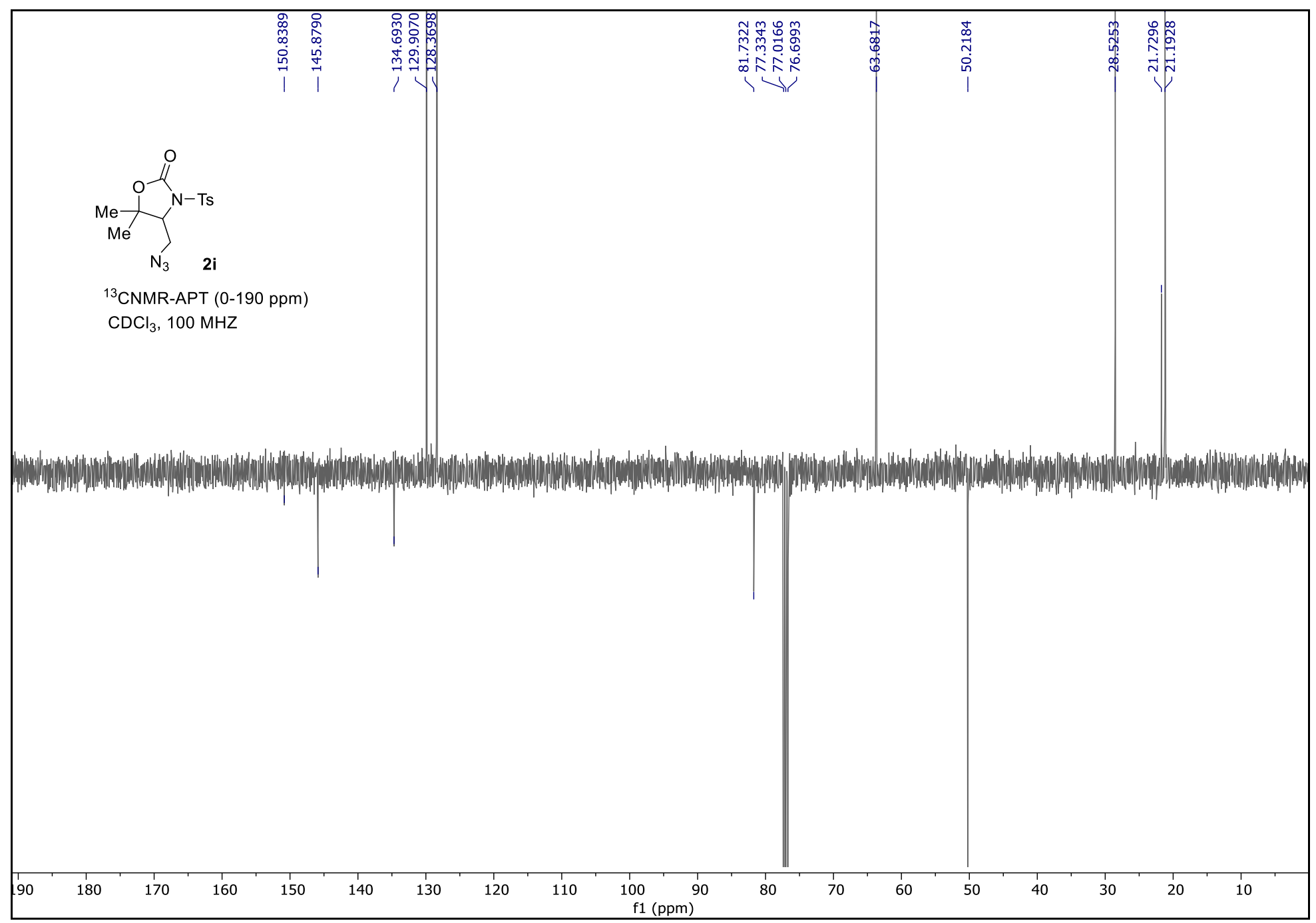


4-(Azidomethyl)-1-methyl-3-tosylimidazolidin-2-one (5a)

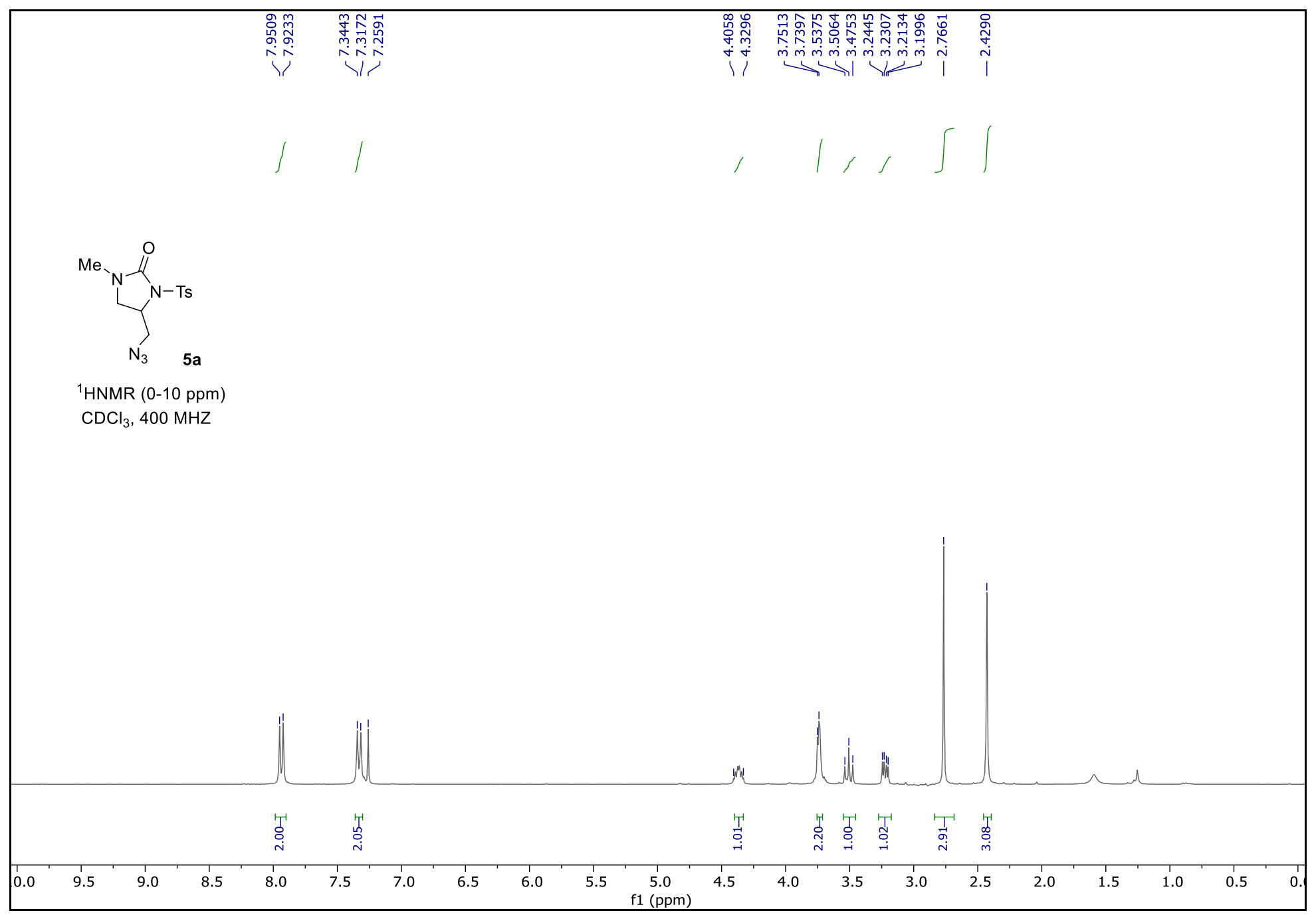




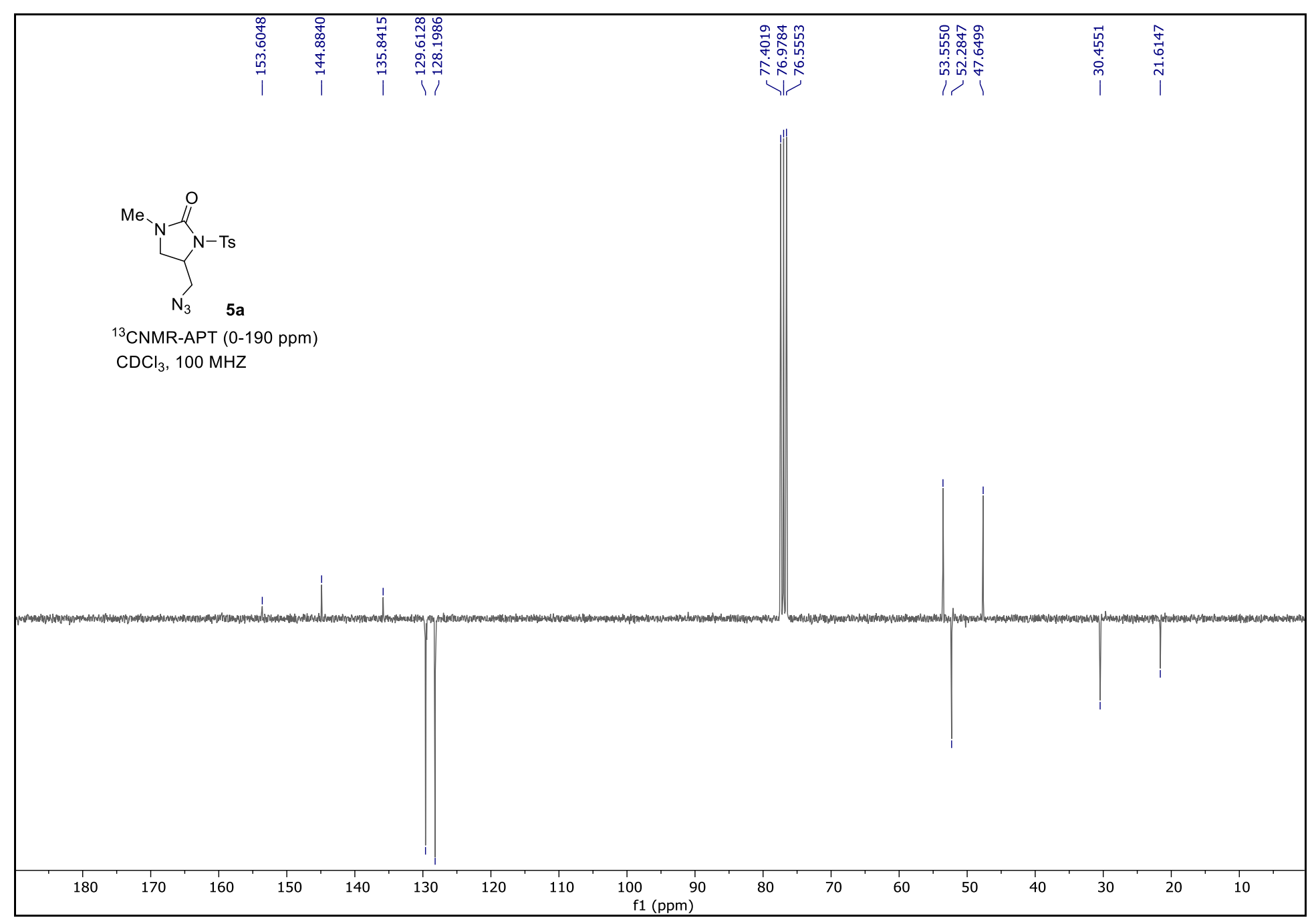


4-(Azidomethyl)-1-cyclohexyl-3-tosylimidazolidin-2-one (5b)

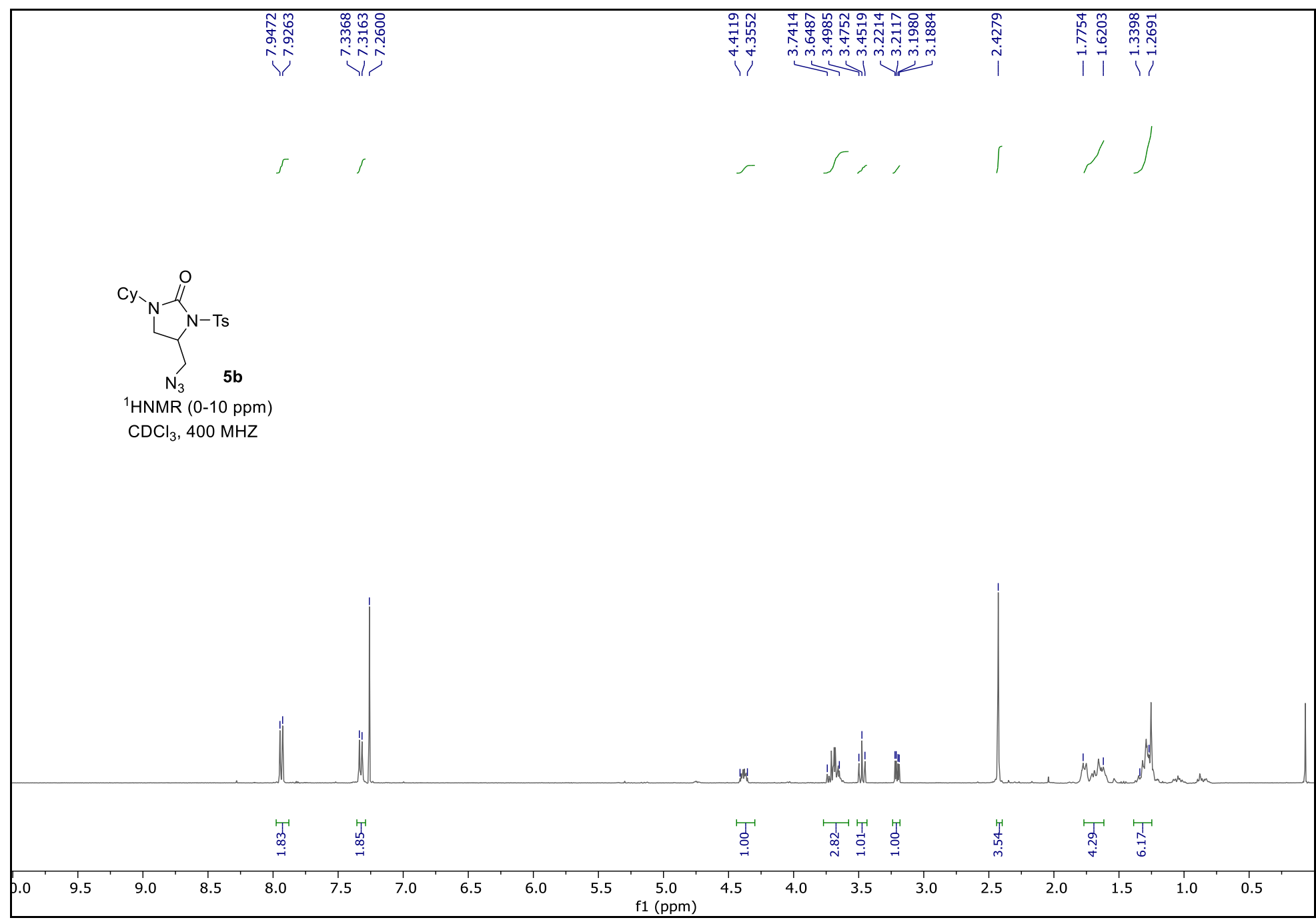




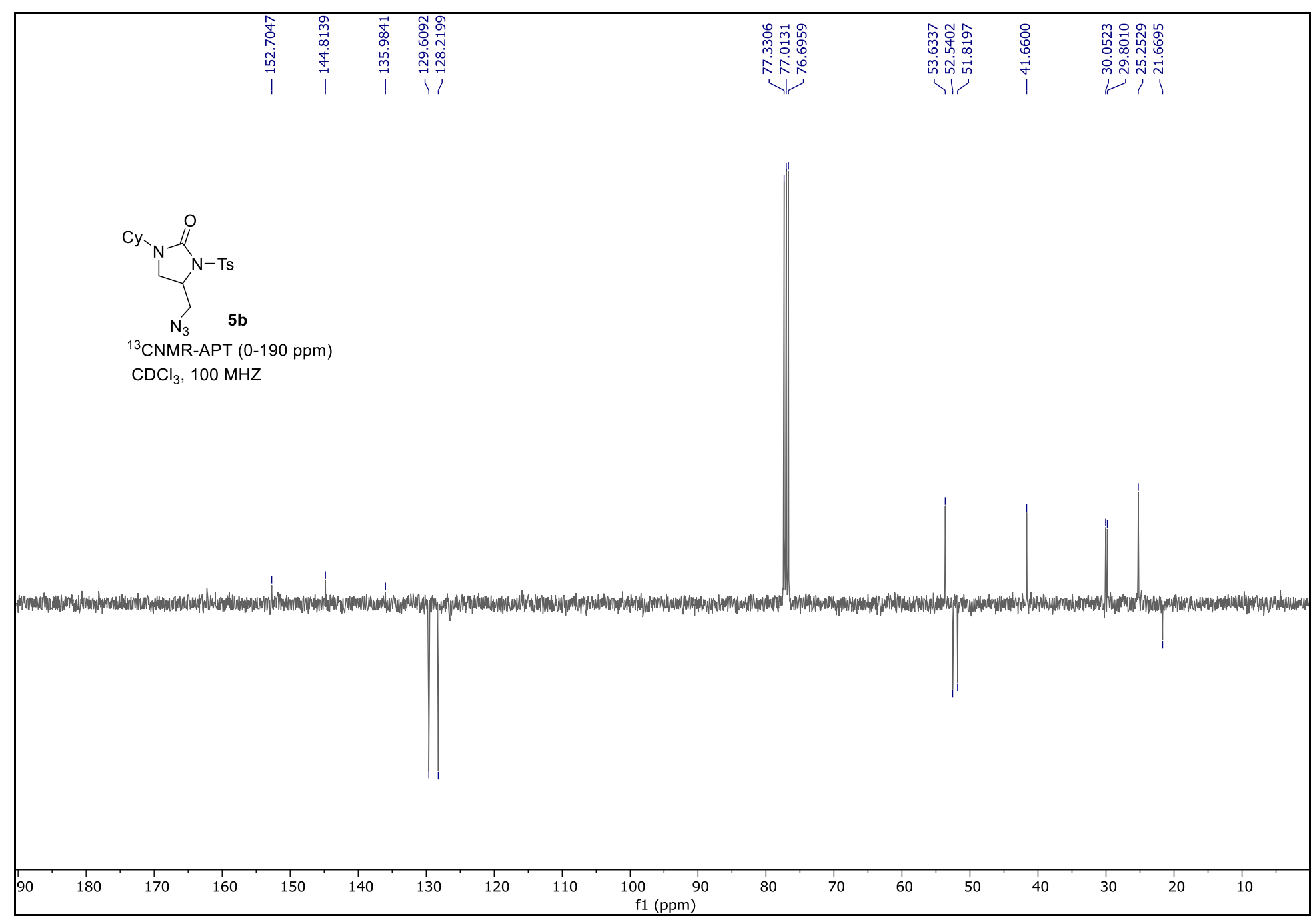


4-(Azidomethyl)-1-phenyl-3-tosylimidazolidin-2-one (5c)

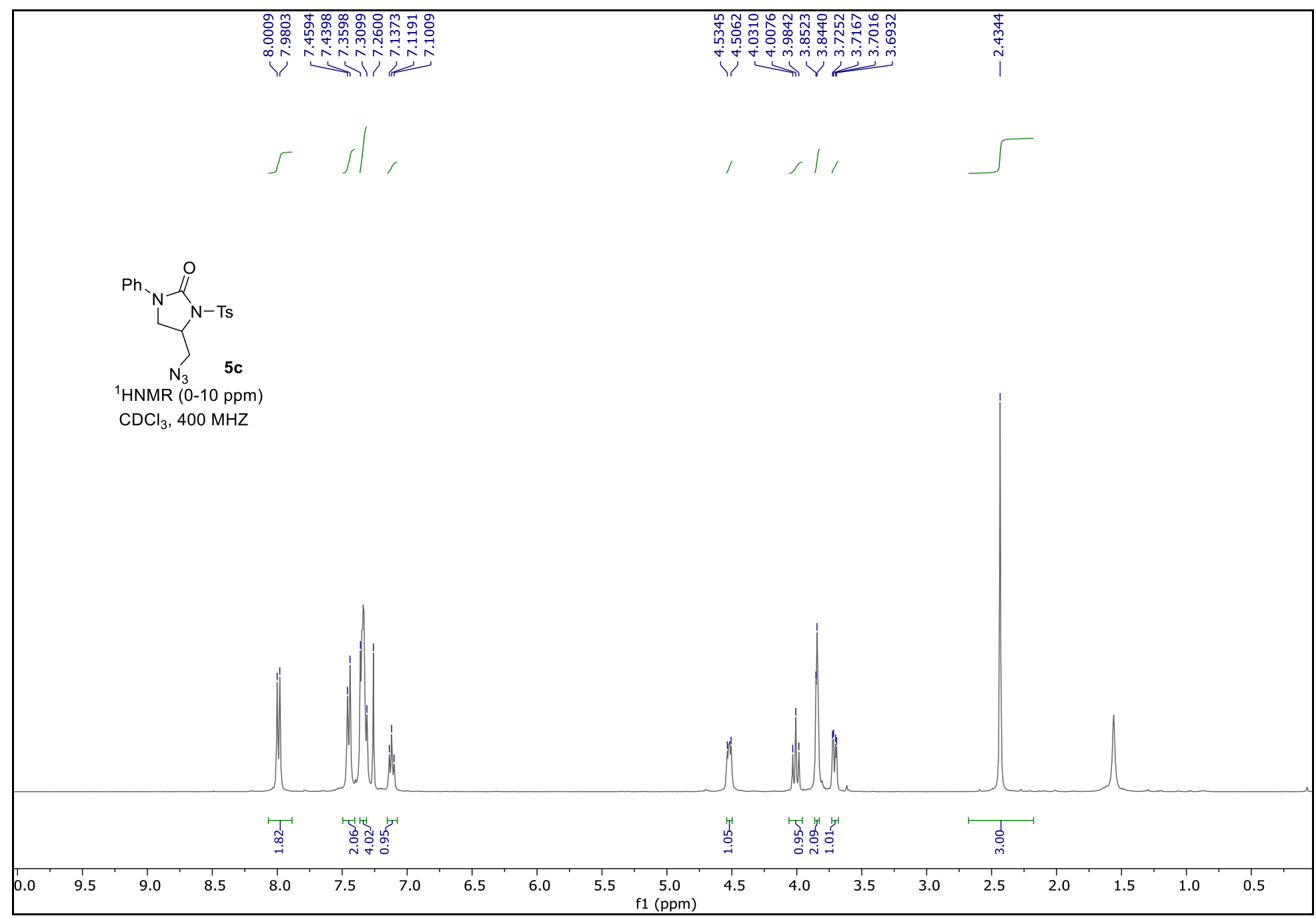




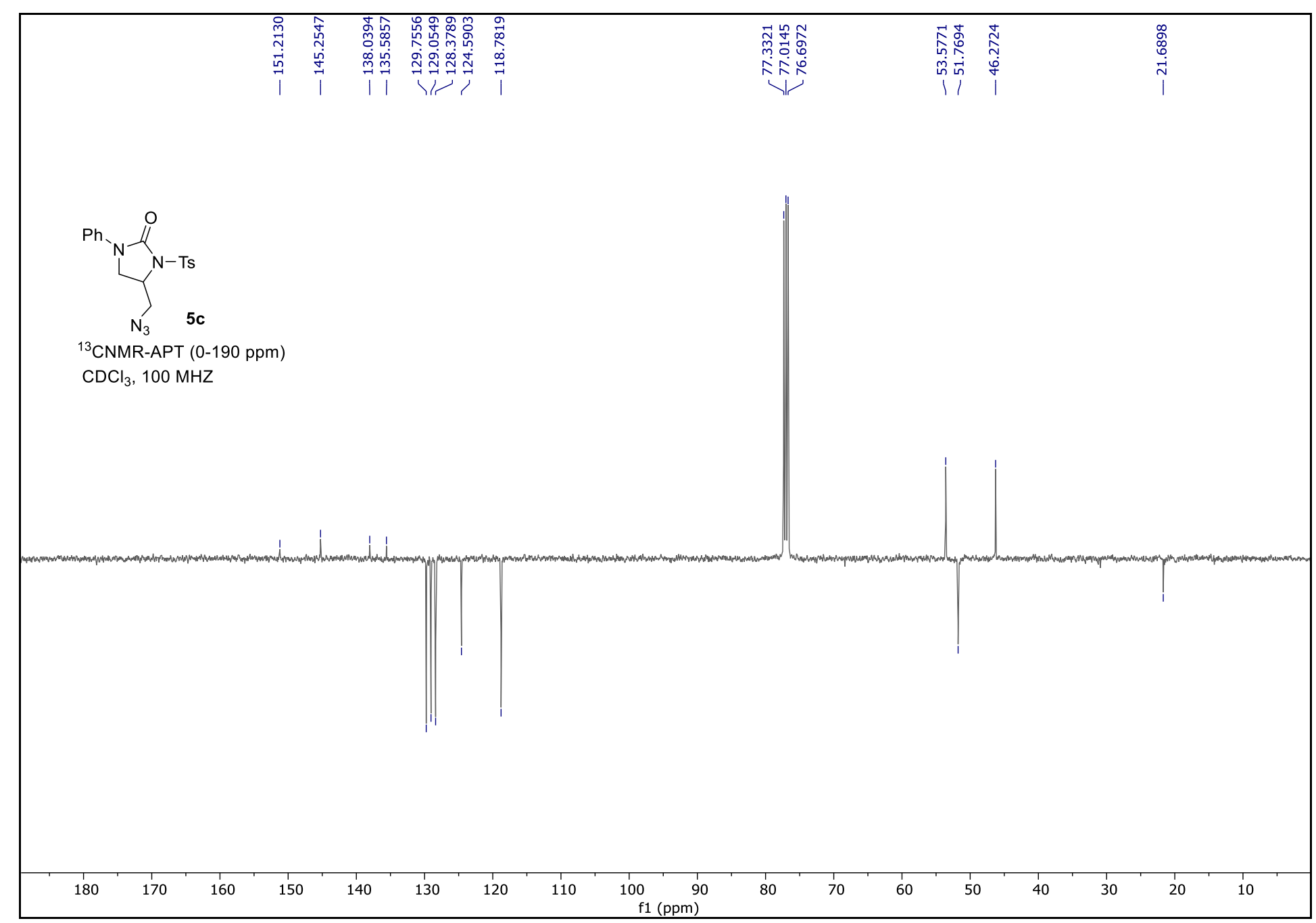


4-(Hydroxymethyl)-1-phenyl-3-tosylimidazolidin-2-one (6c)

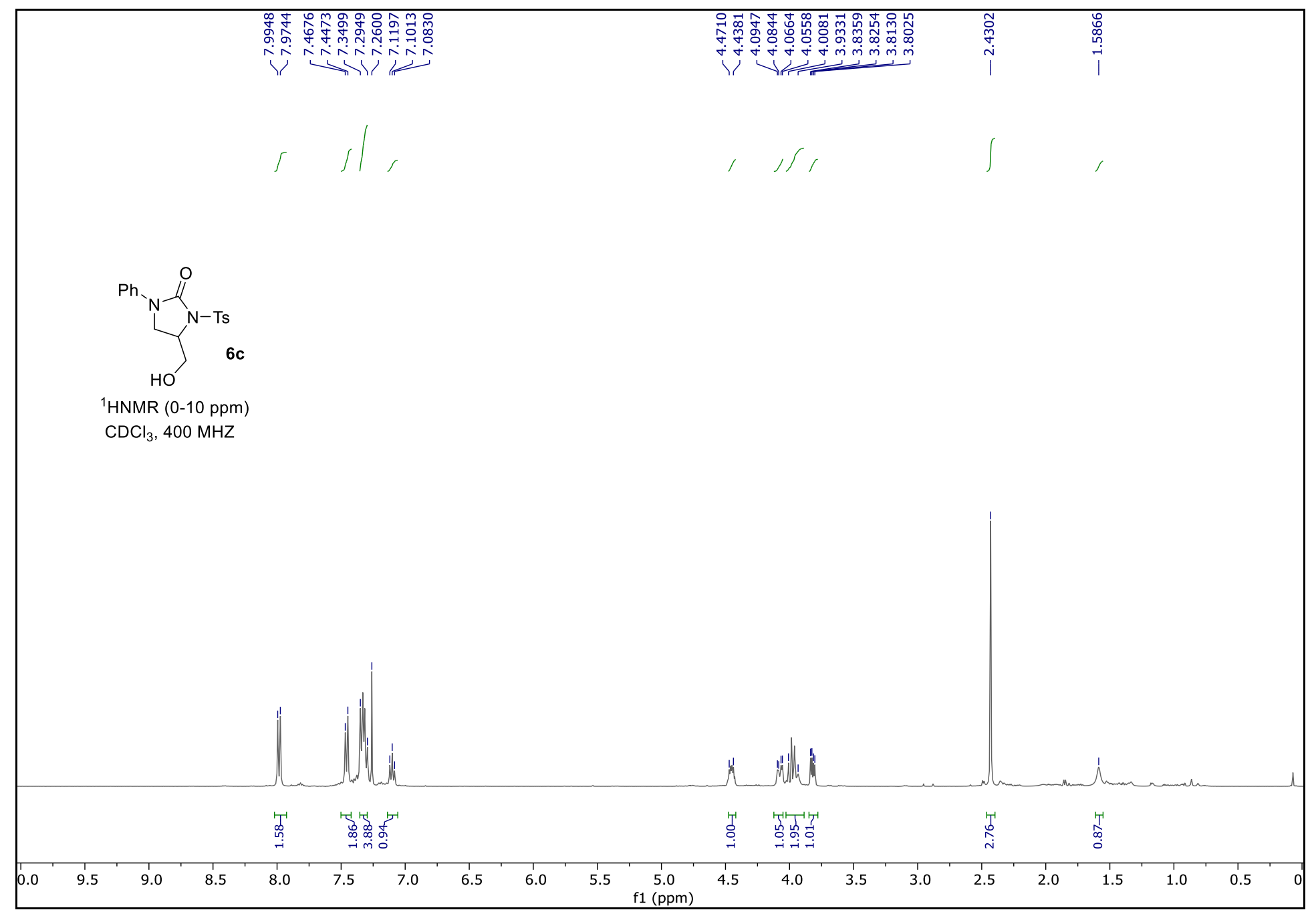




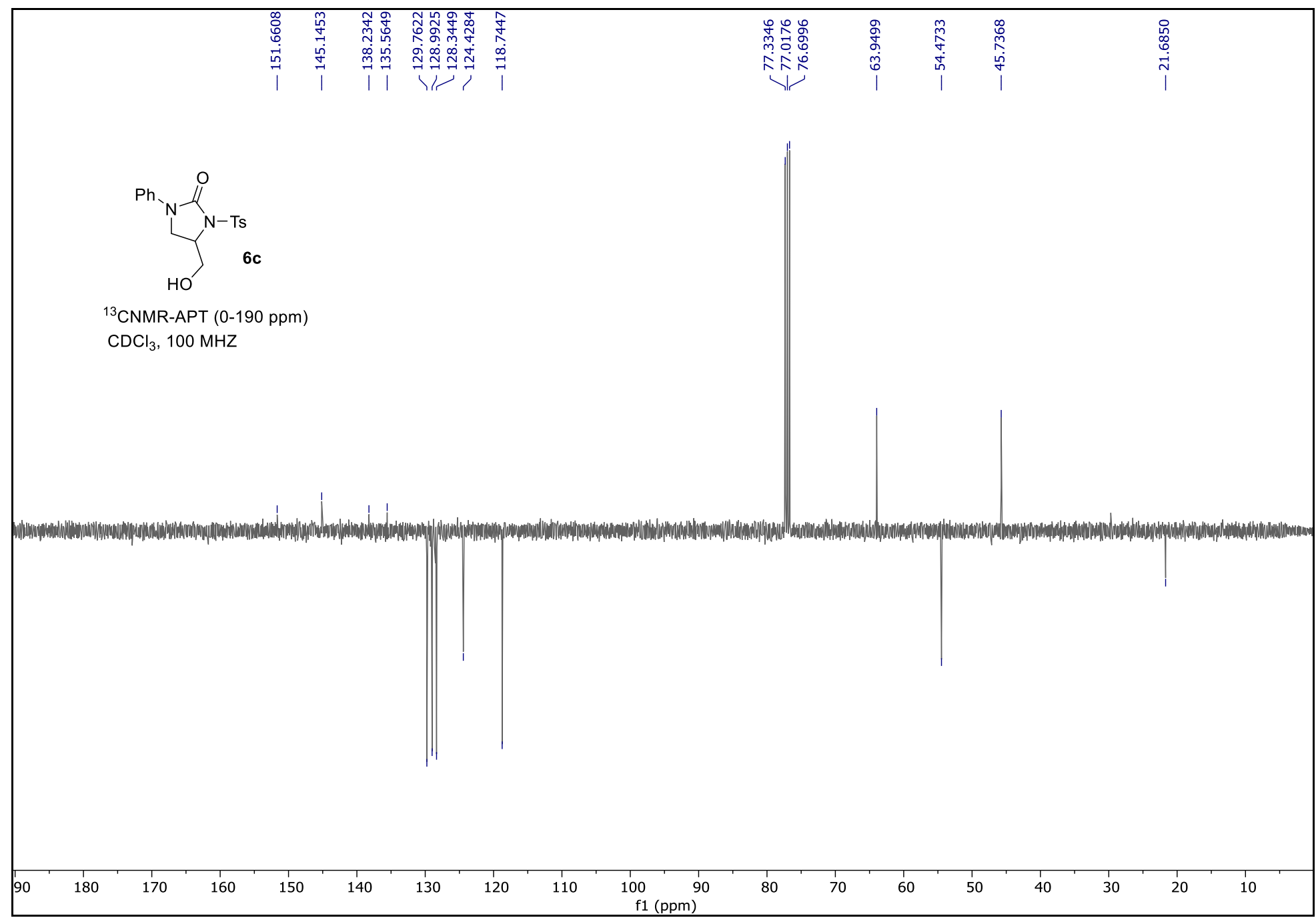


$(R, R)$-4-(Azidomethyl)-1-(1-phenylethyl)-3-tosylimidazolidin-2-one (5d)

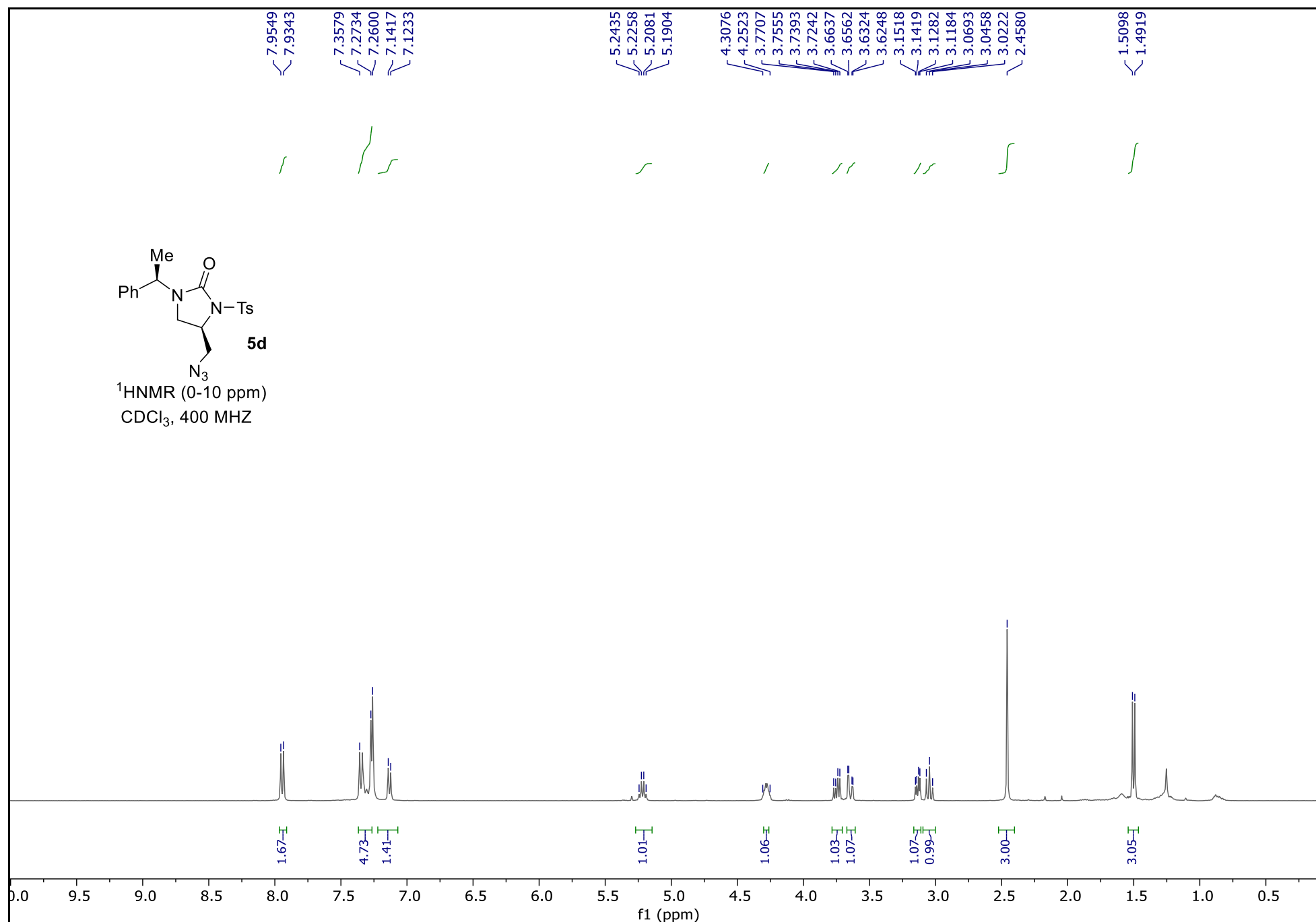




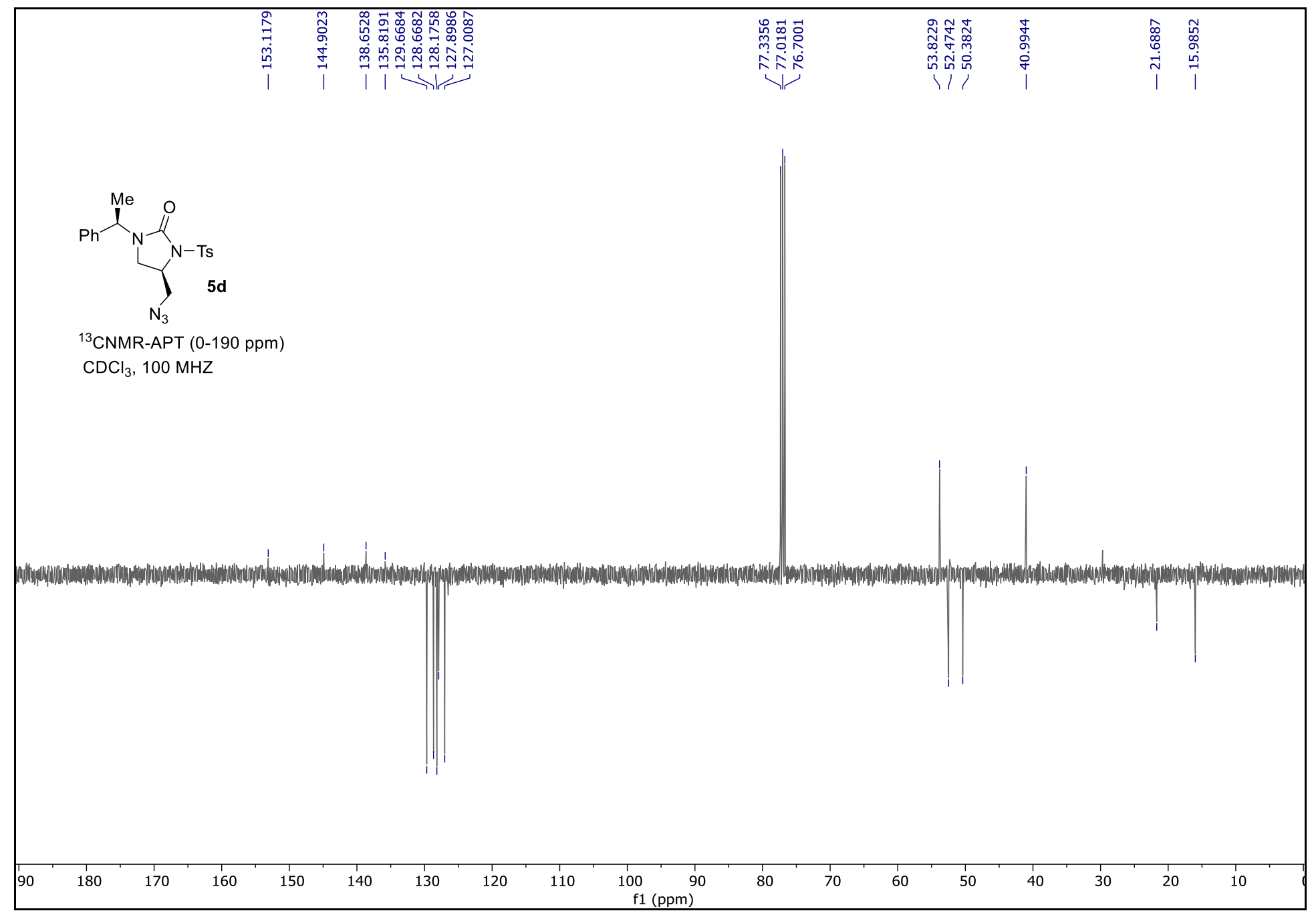




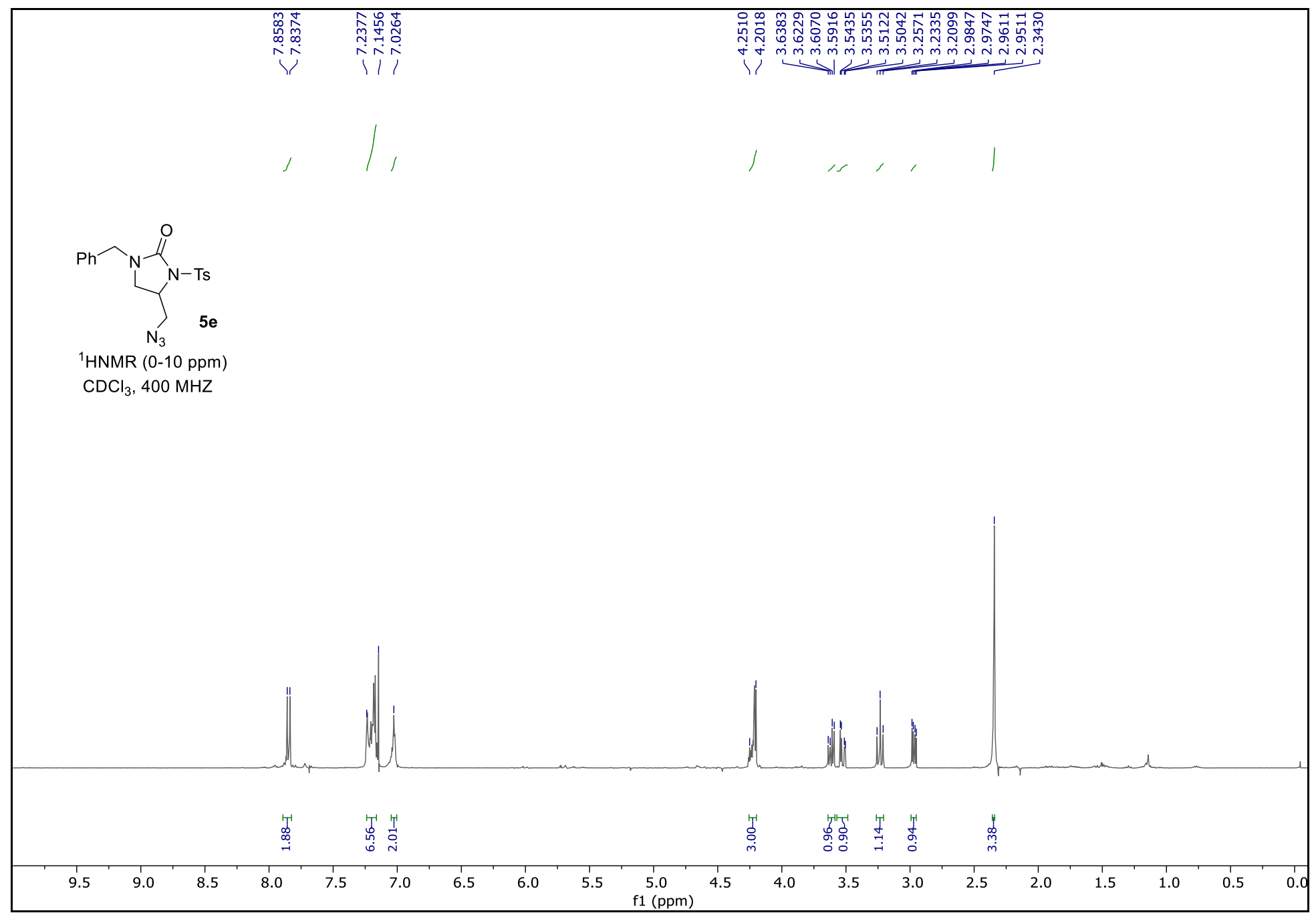




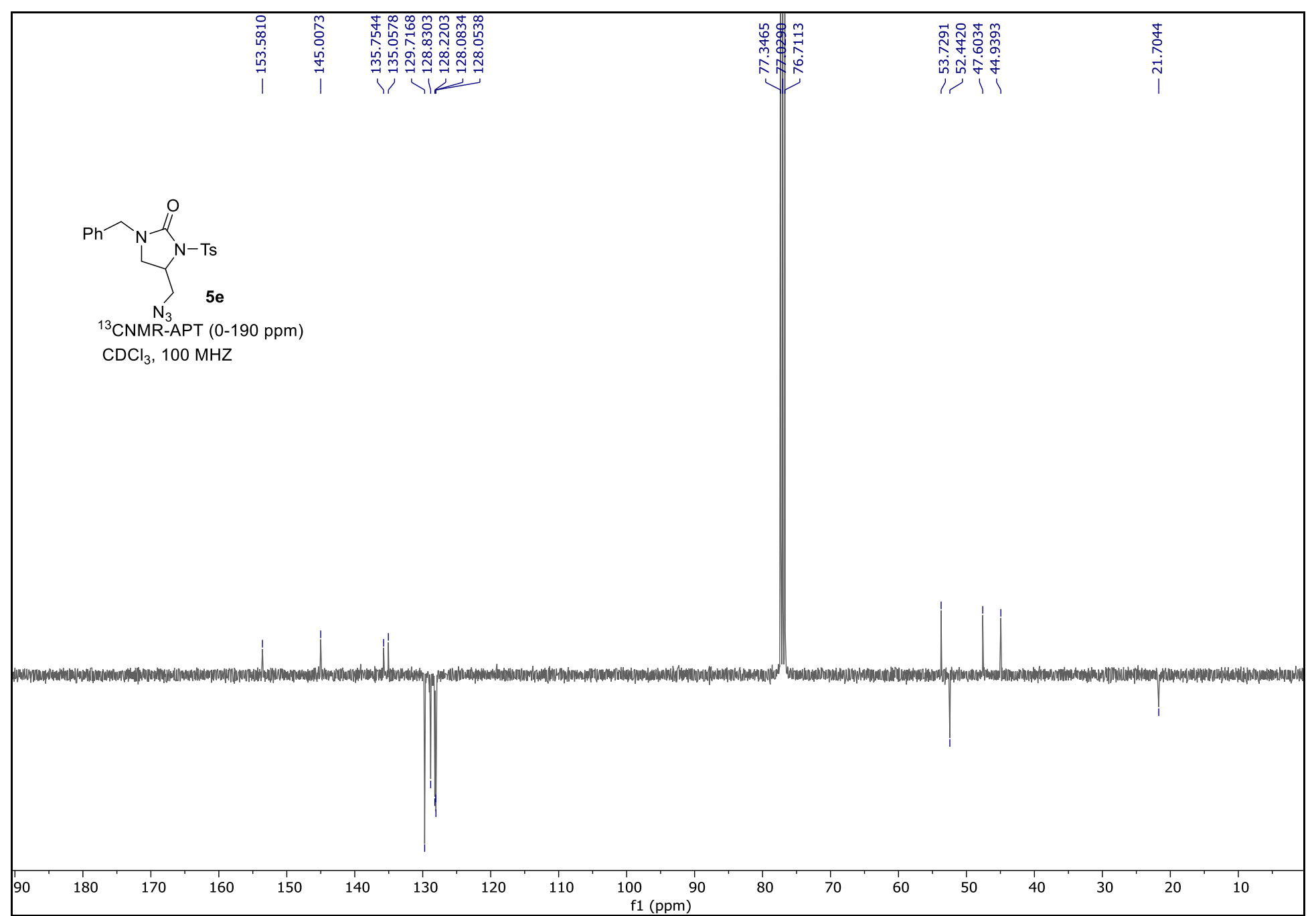


2-(Azidomethyl)-5-methoxy-1-tosylindoline (8b)

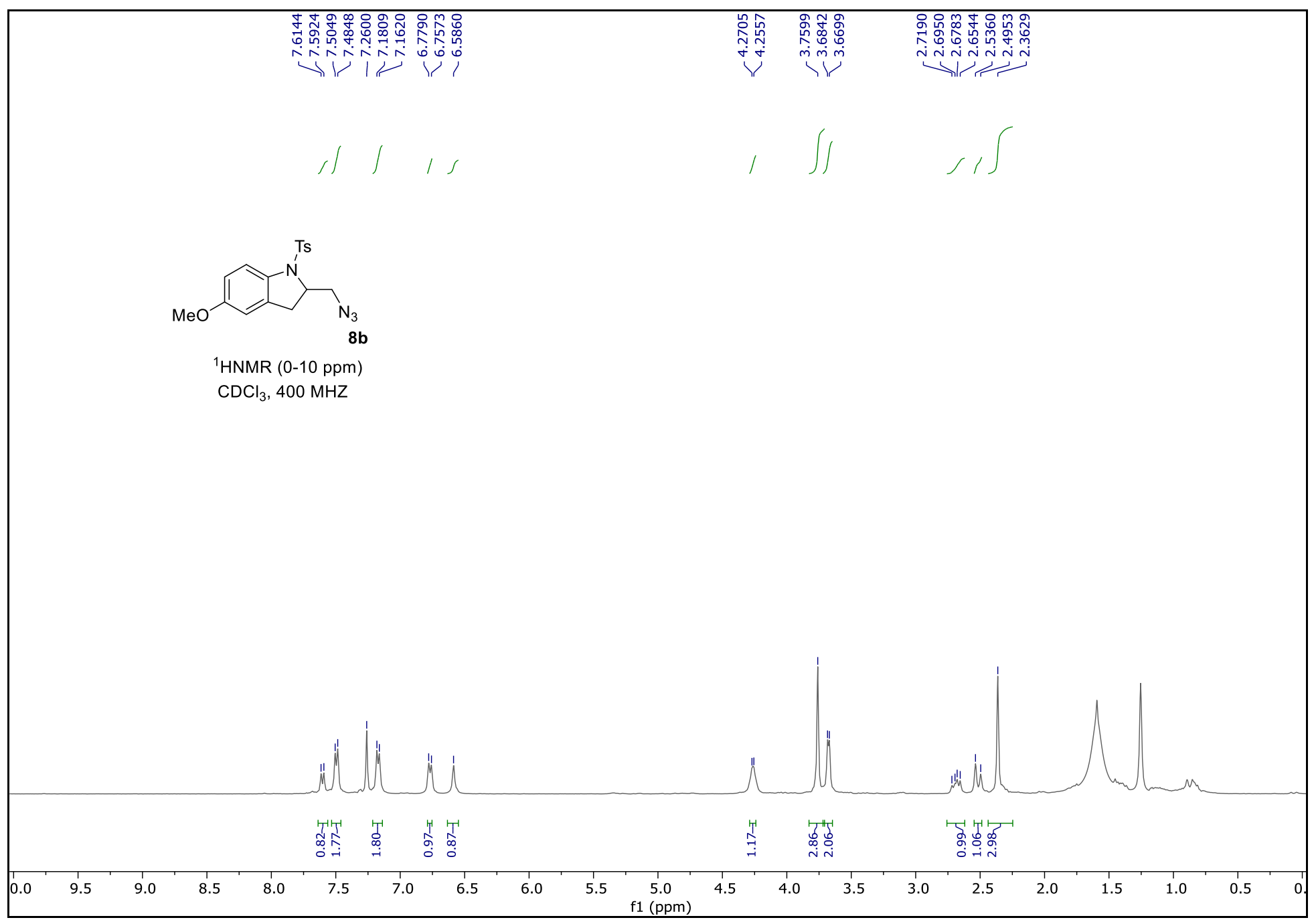




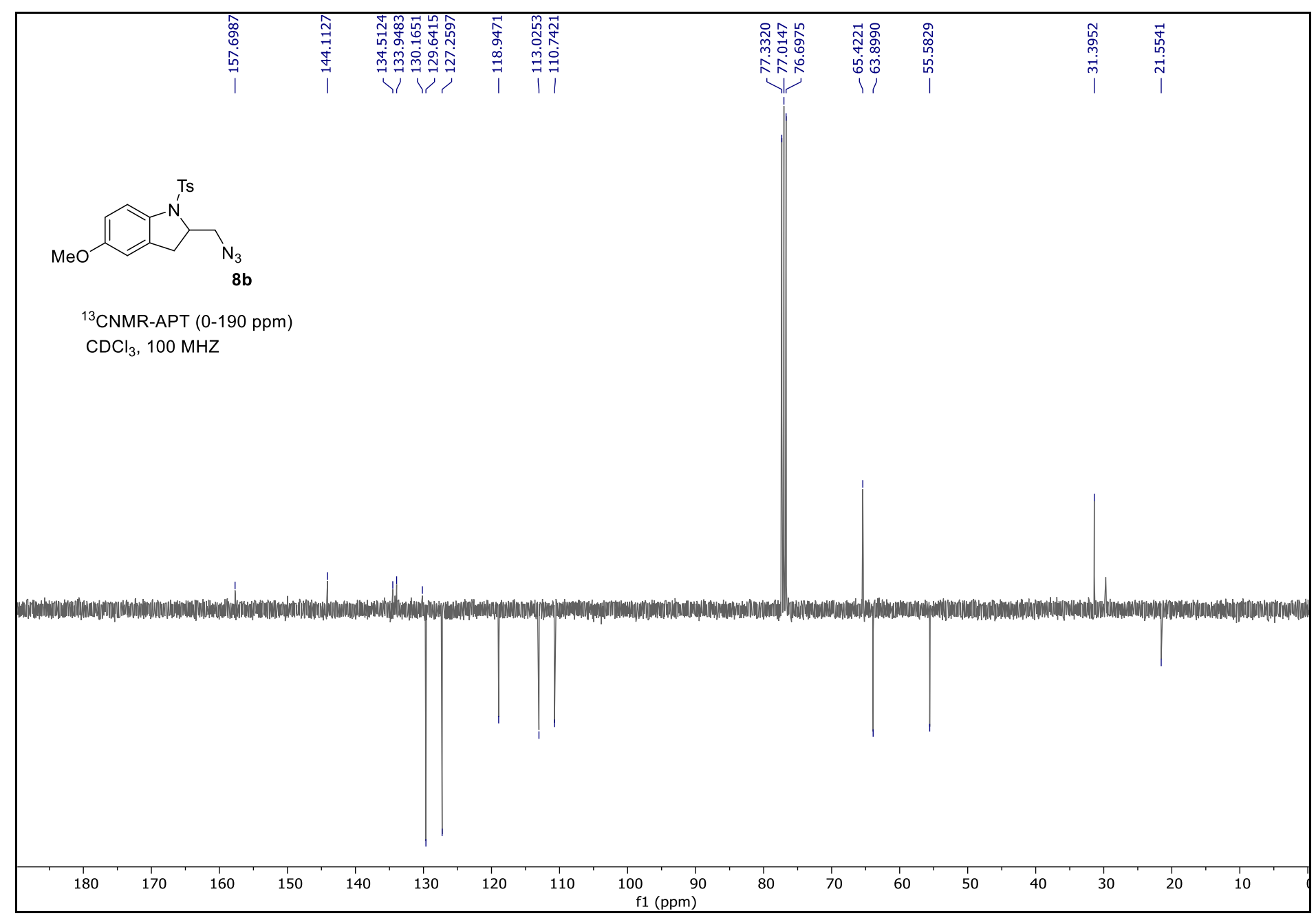


5-(Azidomethyl)-1-cyclohexyl-4-tosylpiperazin-2-one (10a)

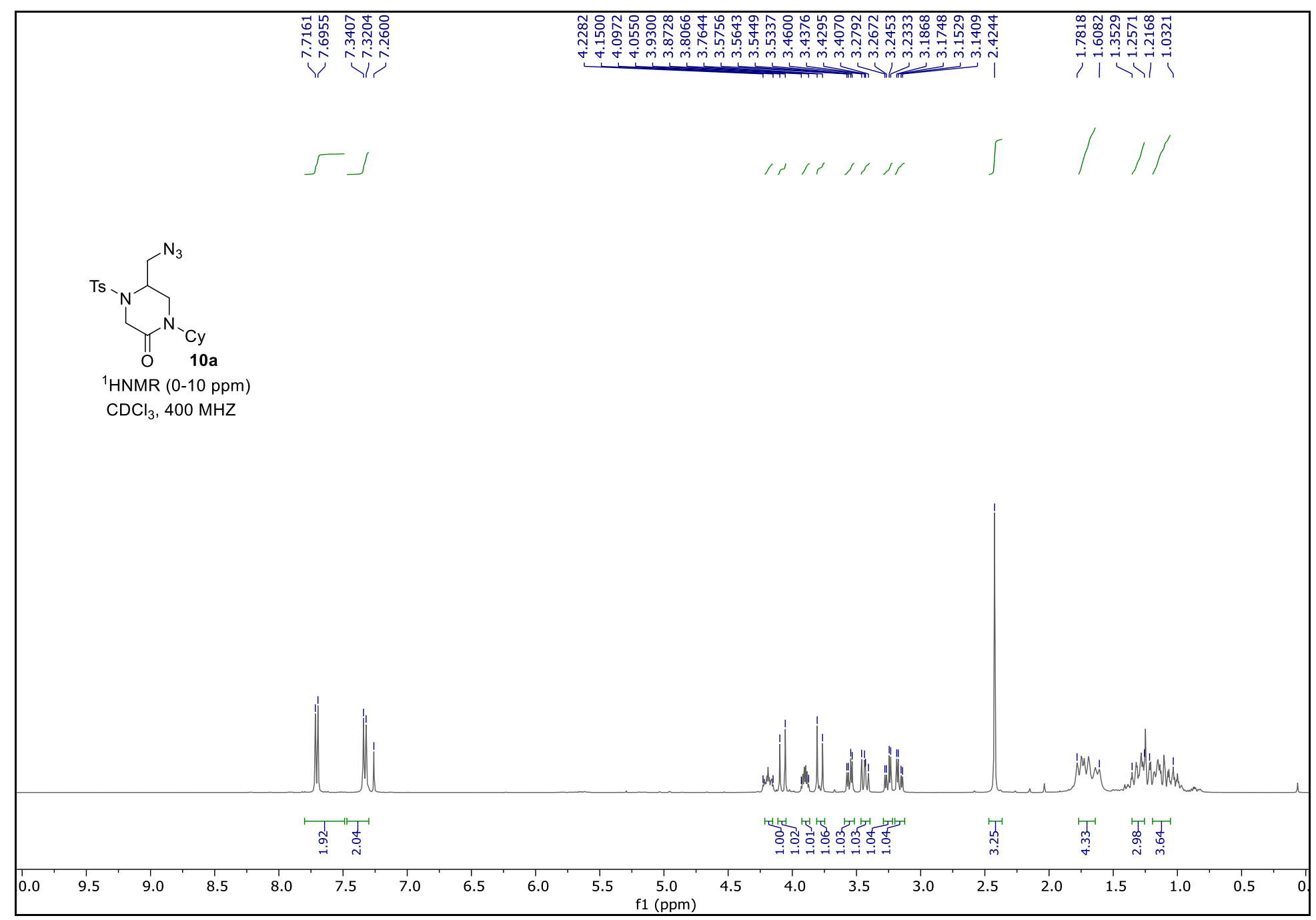




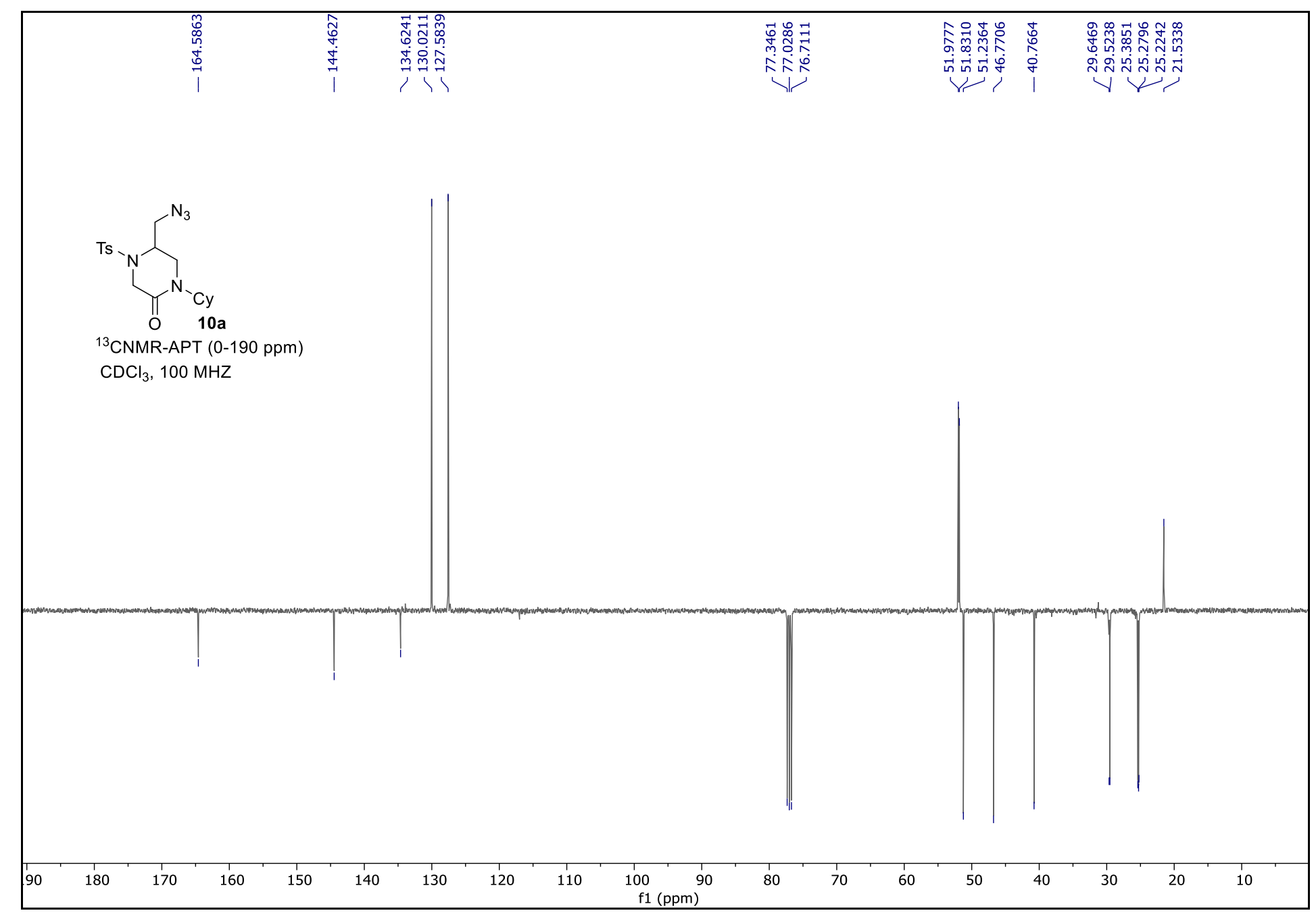


5-(Azidomethyl)-1-phenyl-4-tosylpiperazin-2-one (10b)

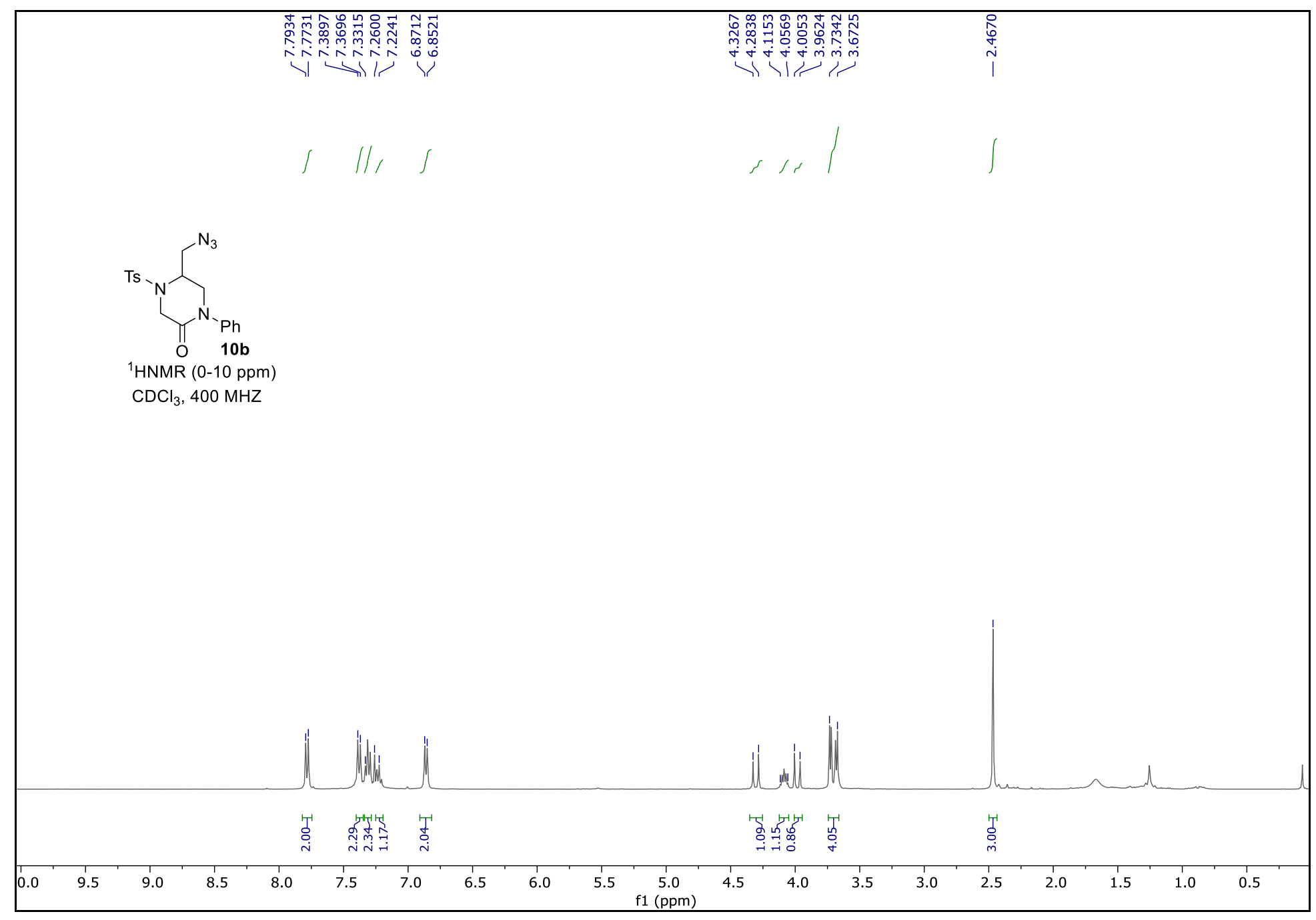




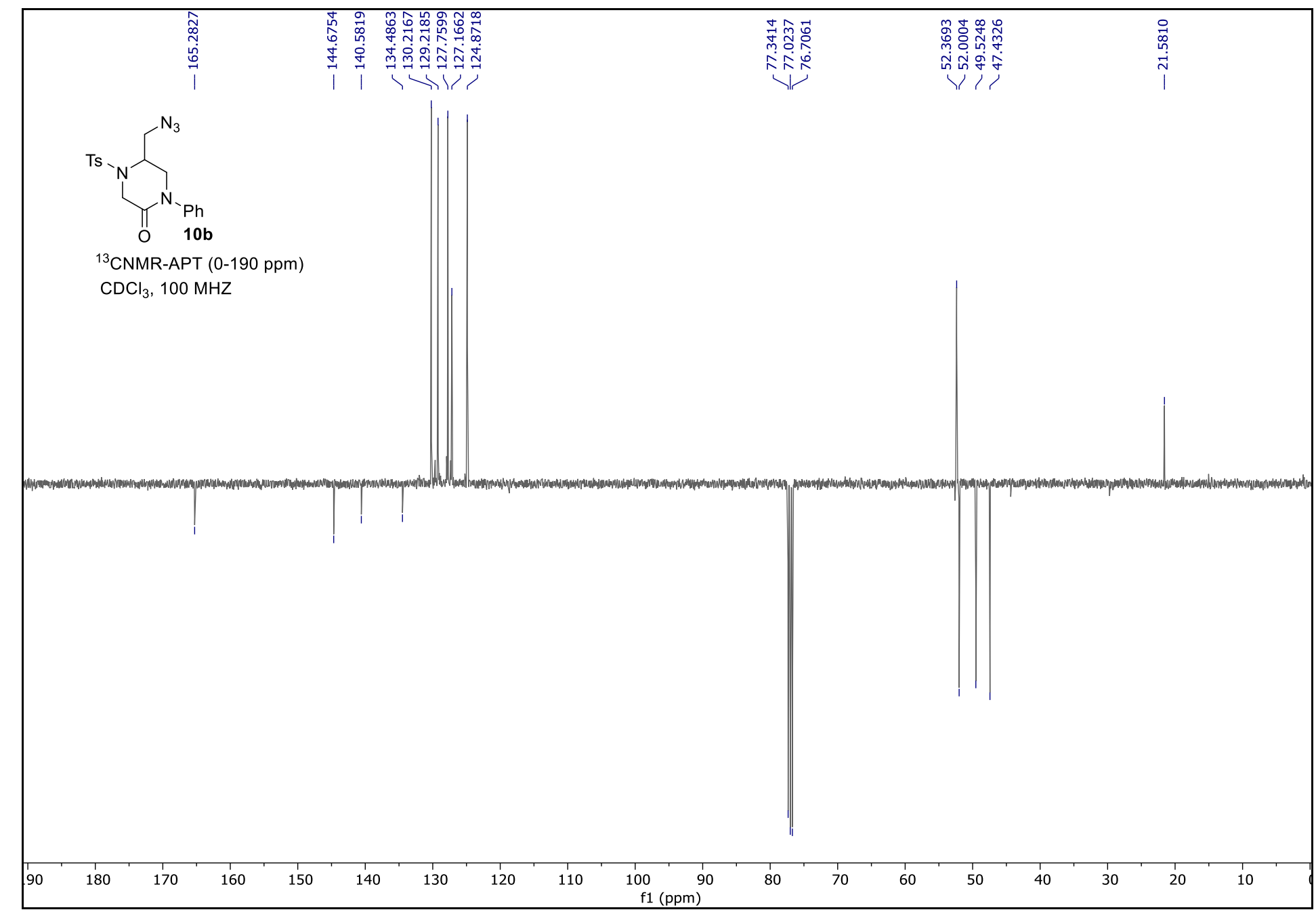


3-(Azidomethyl)-4-tosyl-3,4-dihydro-2H-benzo[b][1,4]oxazine (12)

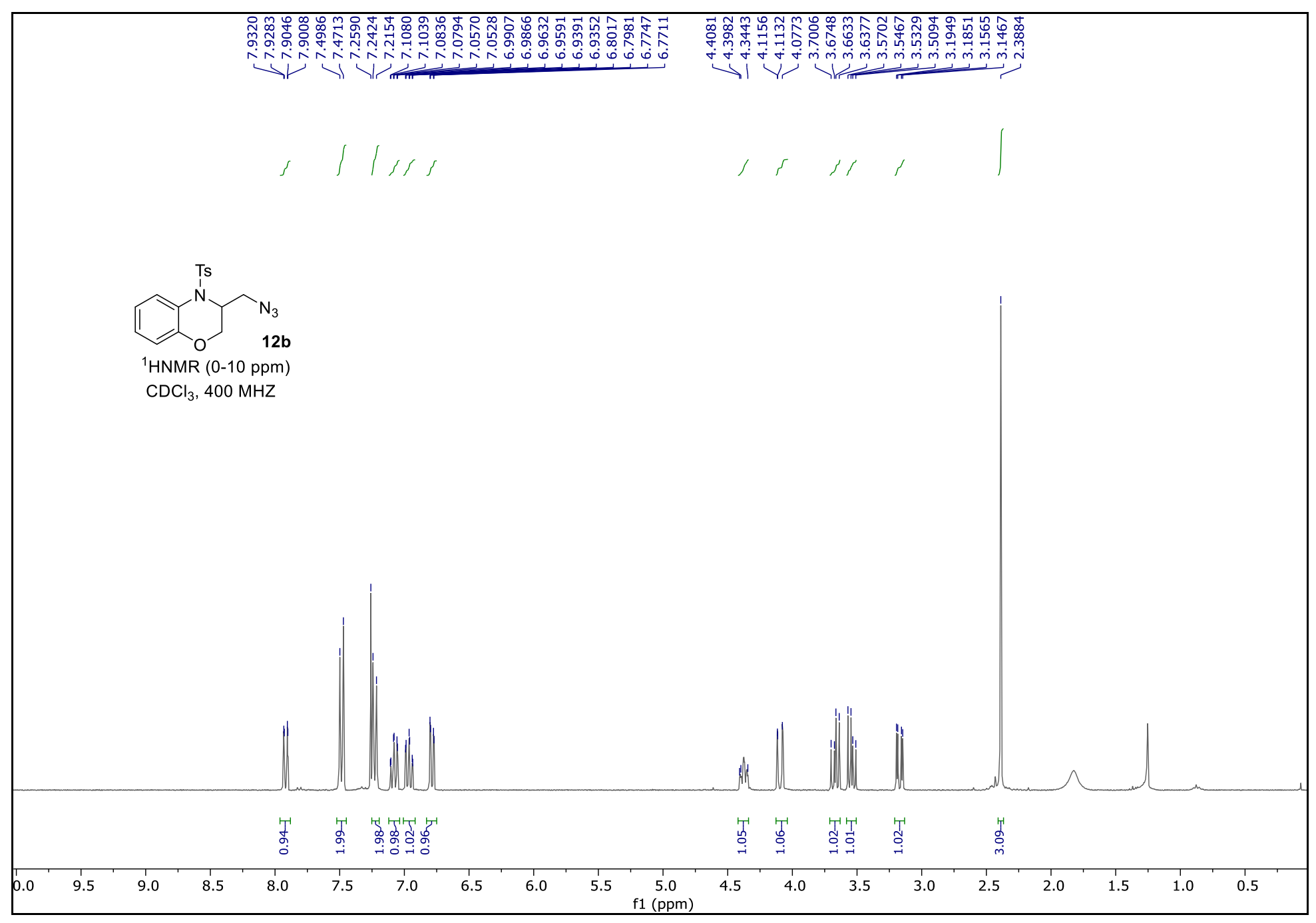




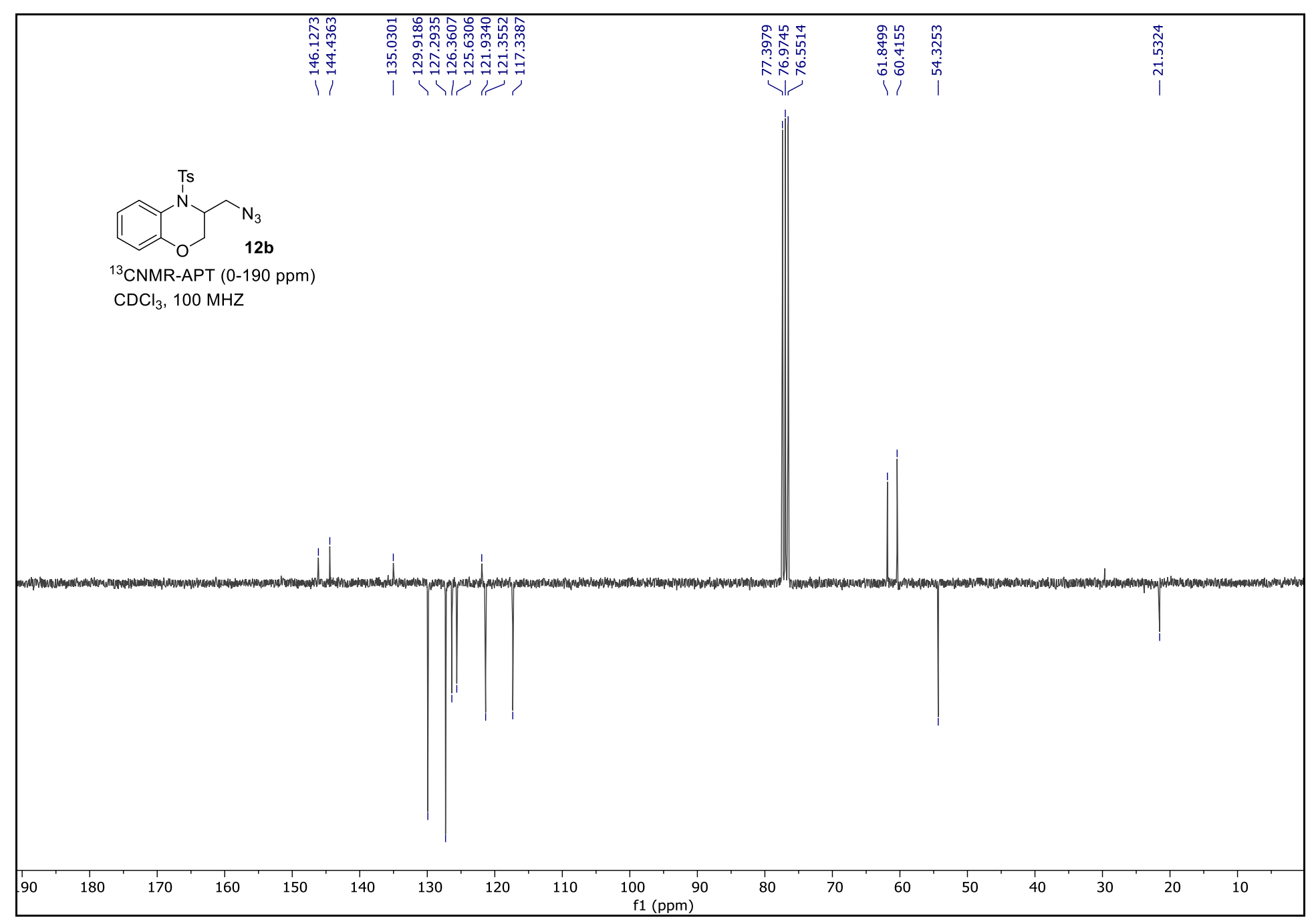


2-(Azidomethyl)-4-methyl-1-tosyl-1,2,3,4-tetrahydro-5H-benzo[e][1,4]diazepin-5-one (14a).

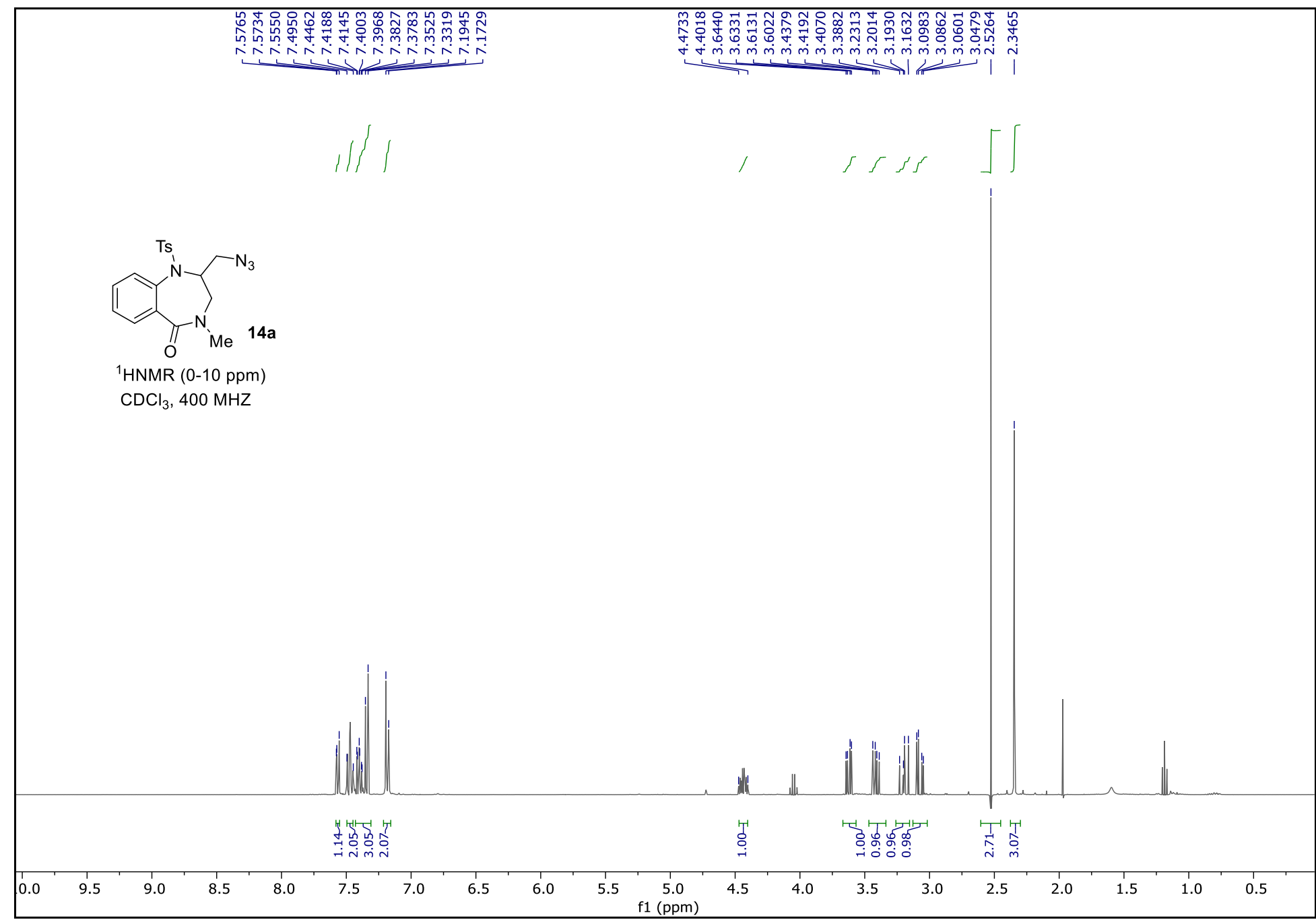




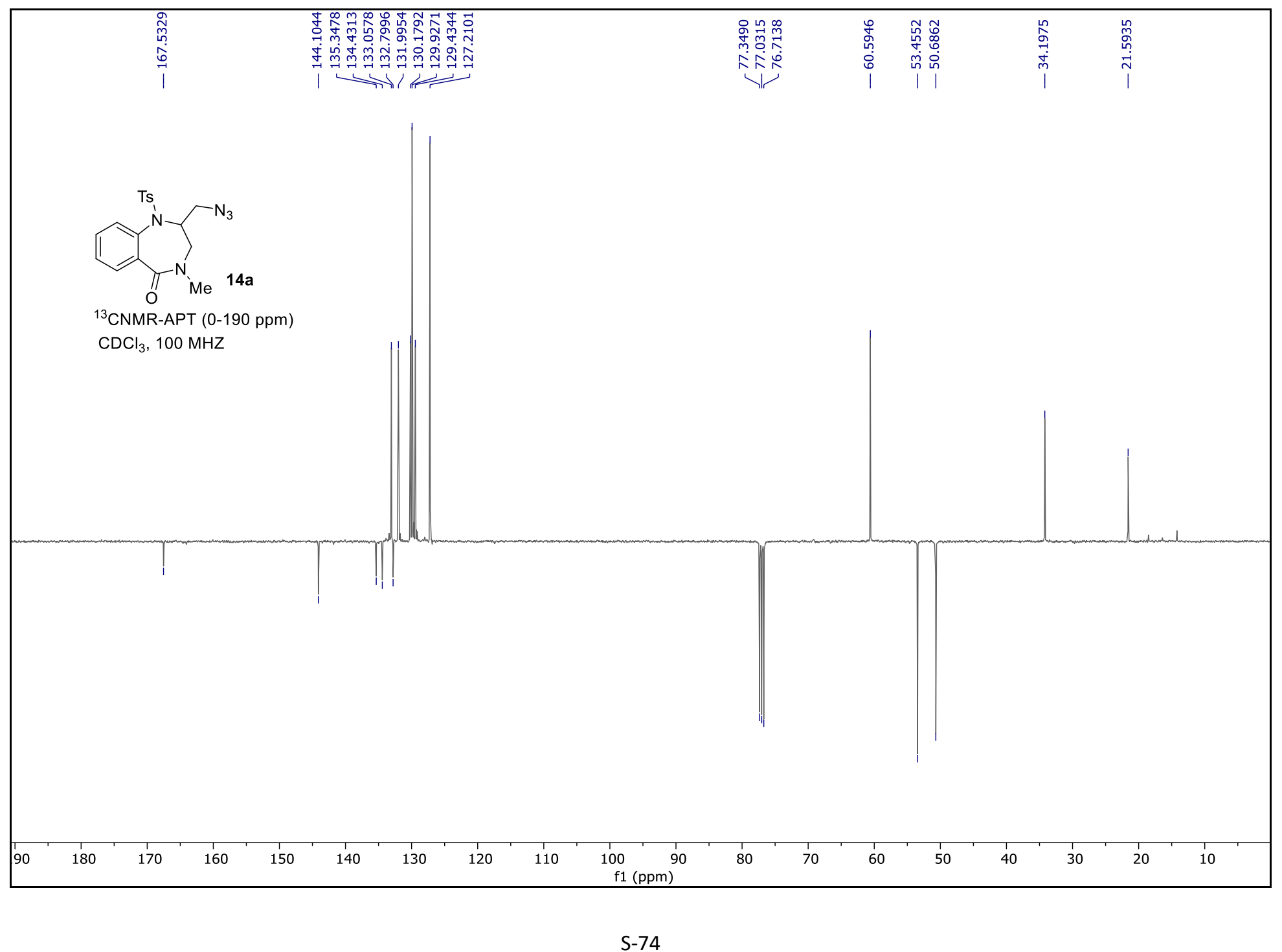




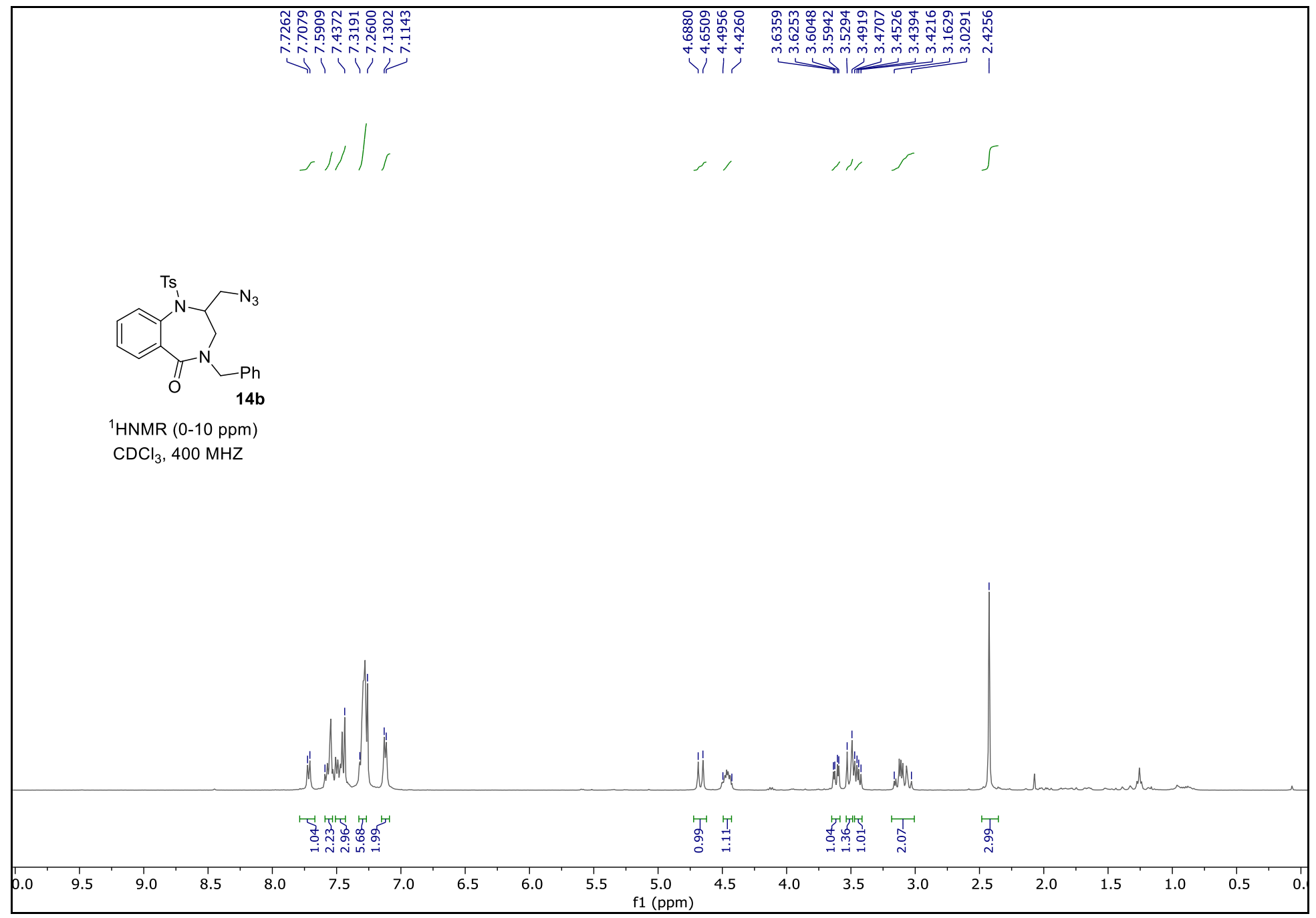




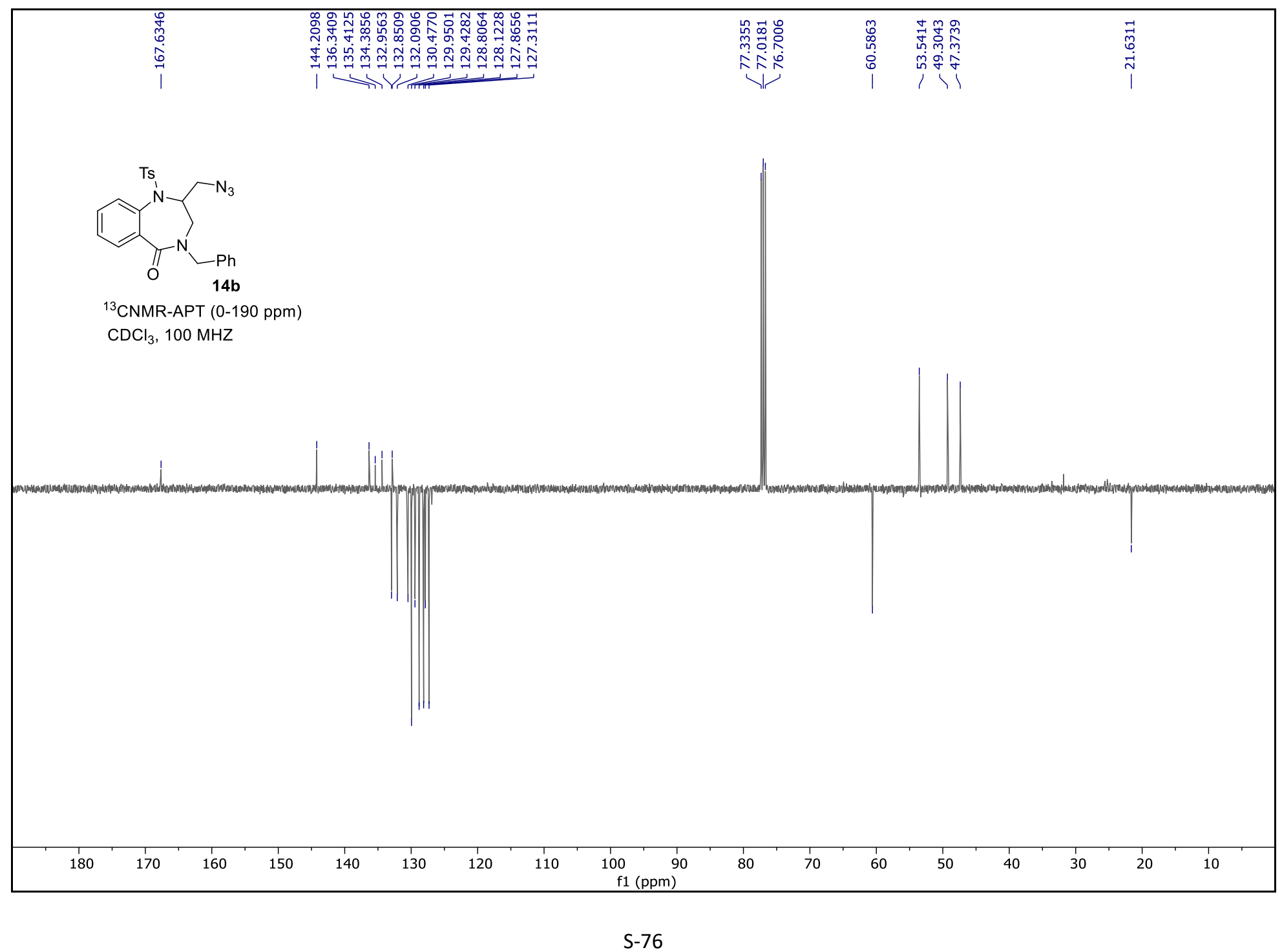


2-(Azidomethyl)-4-cyclohexyl-1-tosyl-1,2,3,4-tetrahydro-5H-benzo[e][1,4]diazepin-5-one (14c)

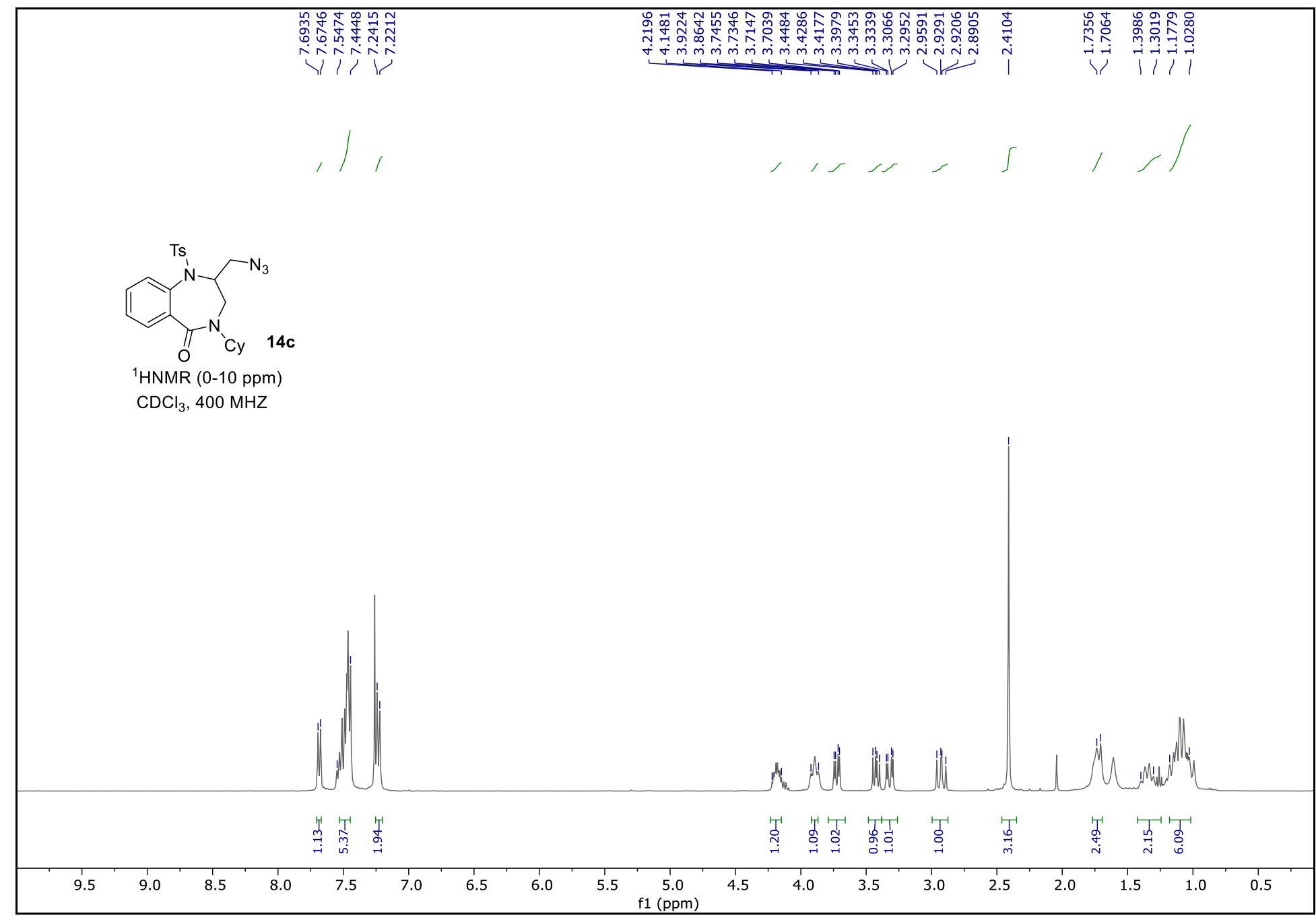




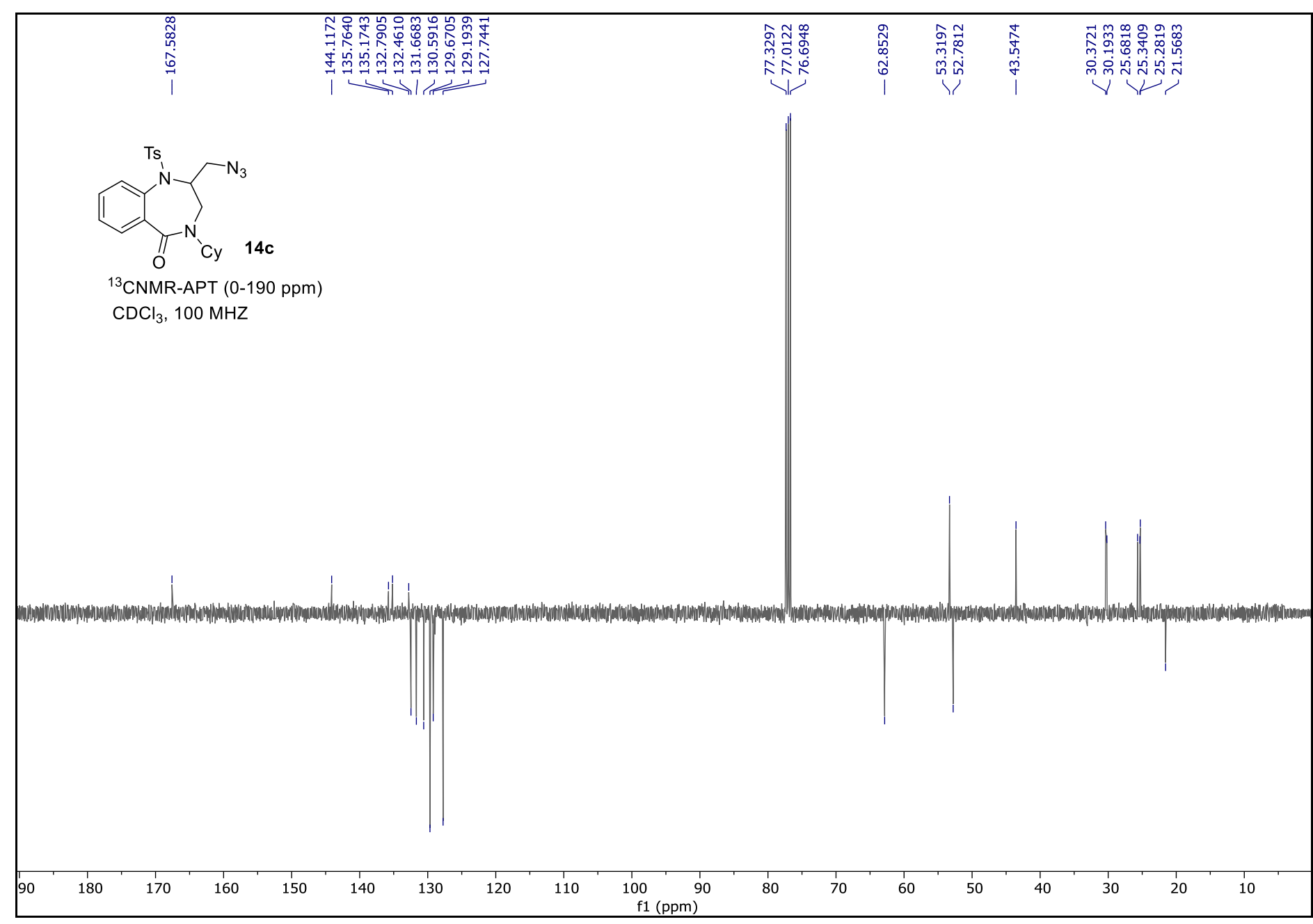




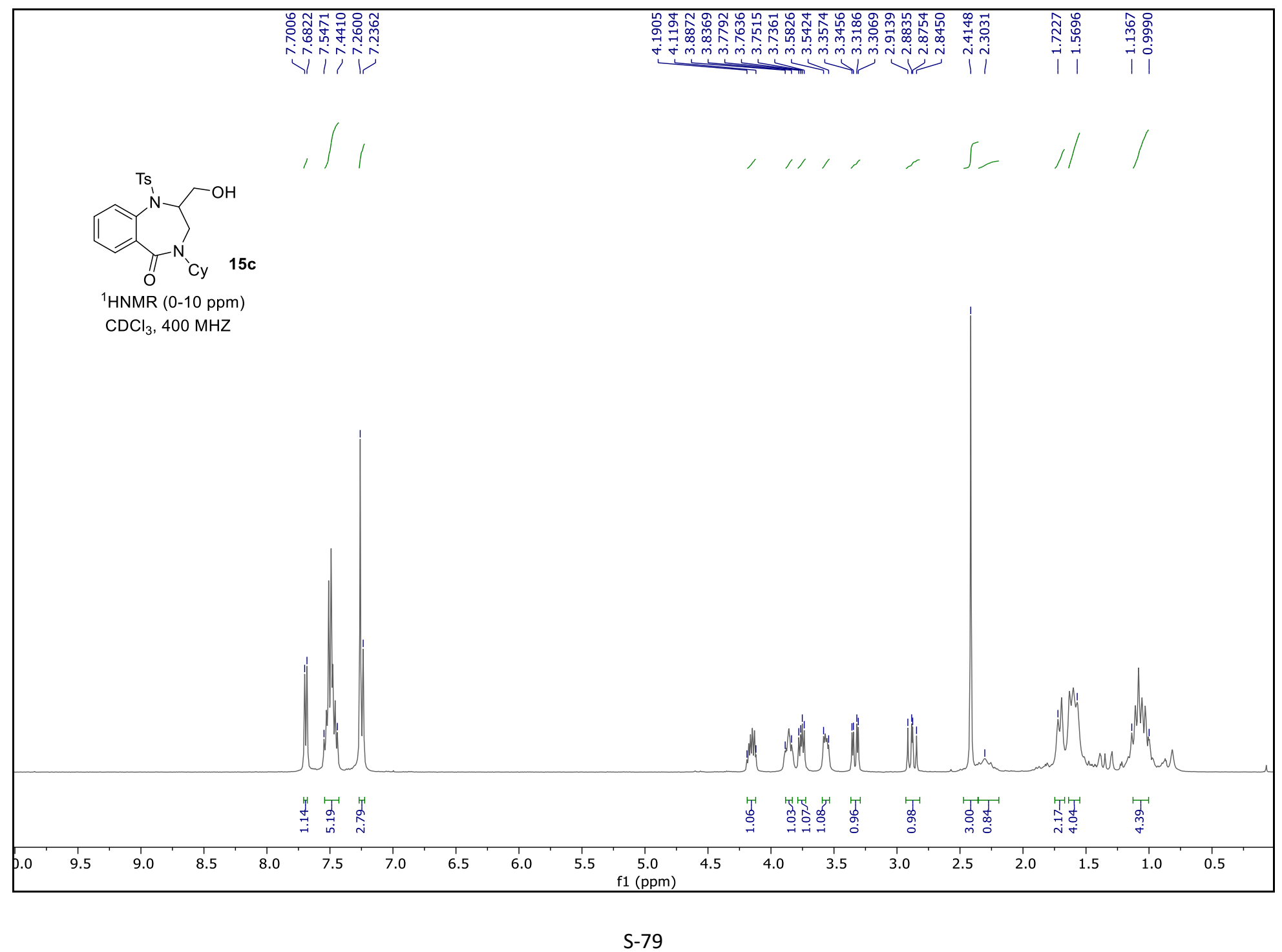




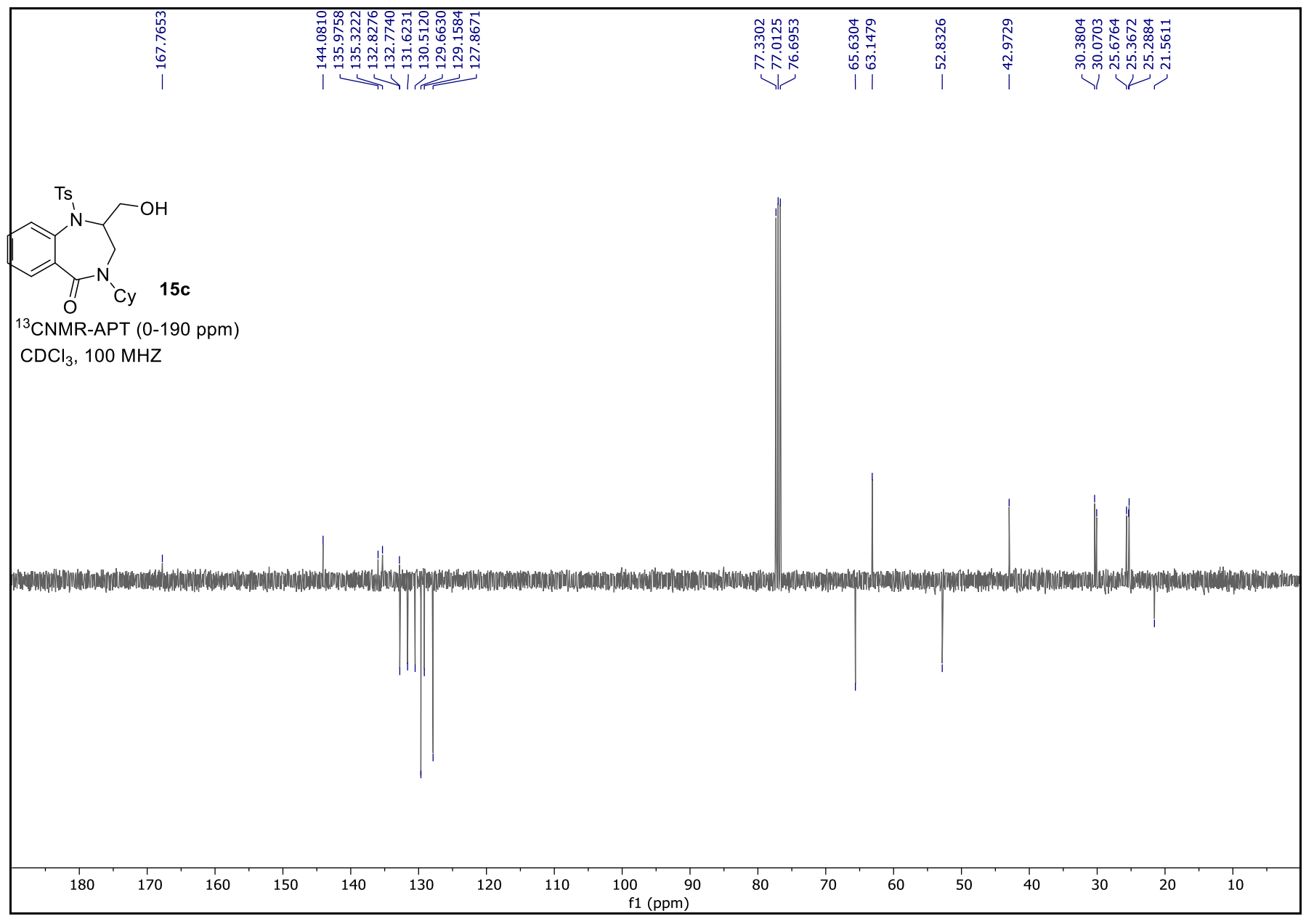


4-(1-Azidoethyl)-3-tosyloxazolidin-2-one (16)

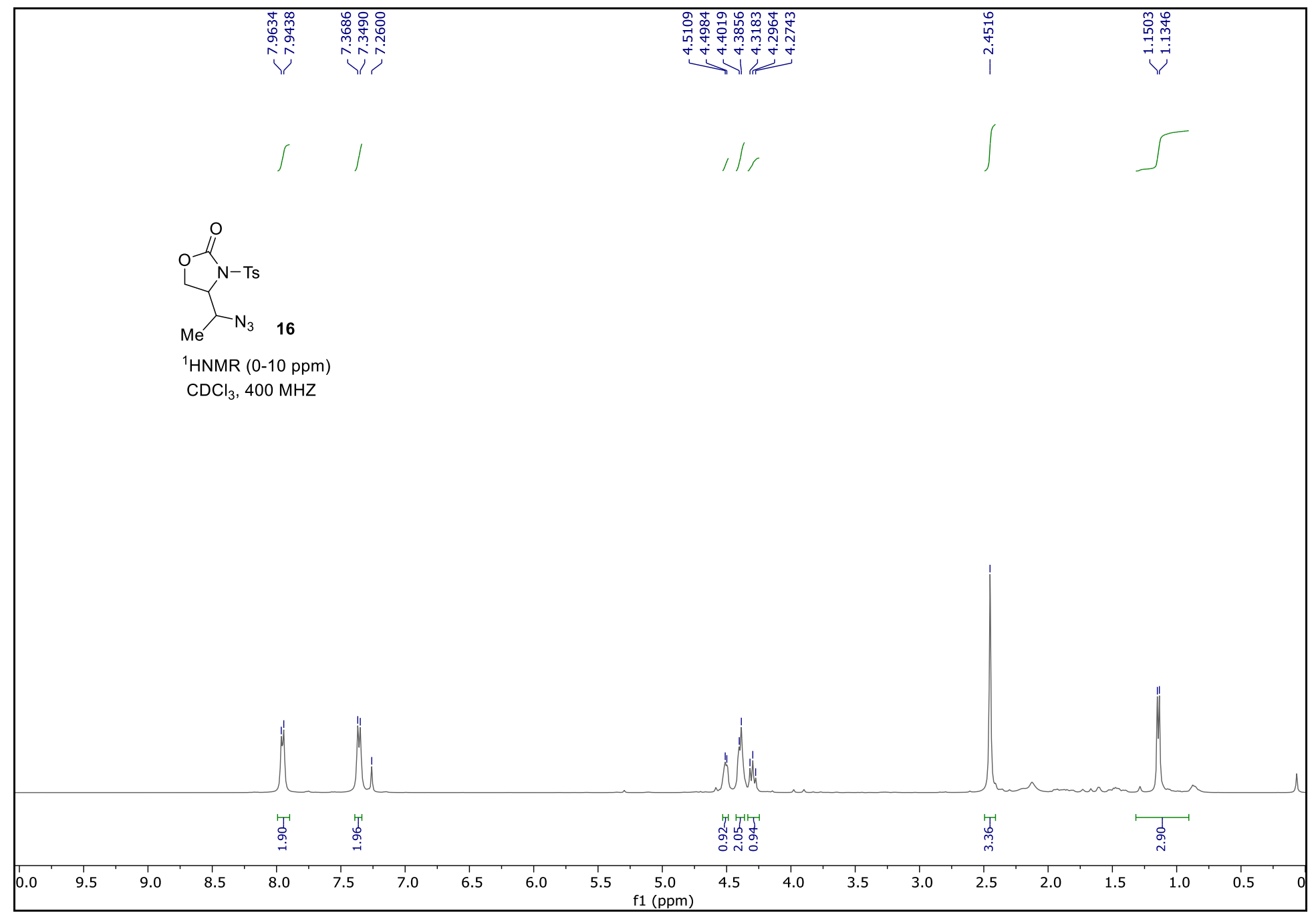

S-81 


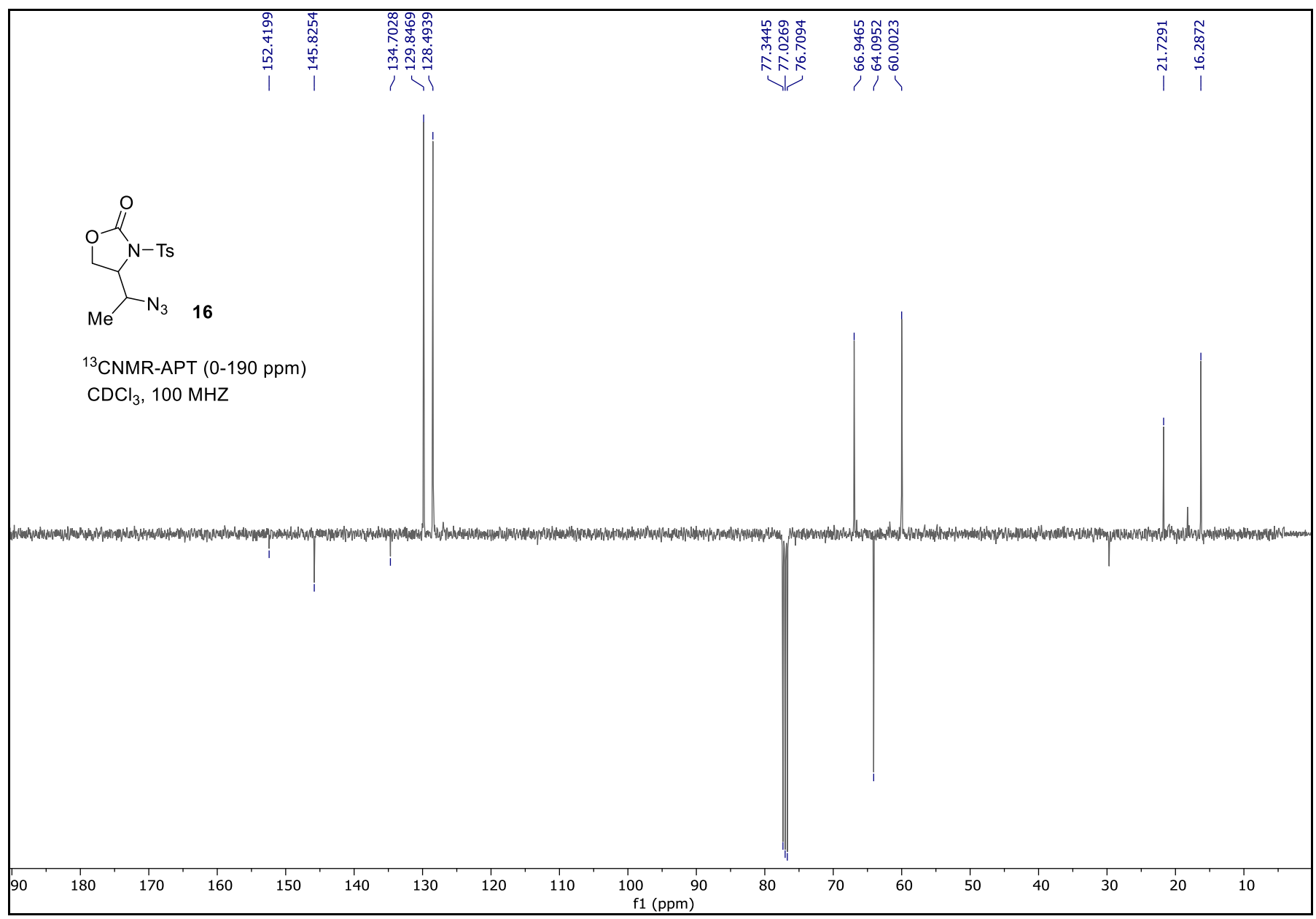

